\title{
Crossed Products by Groupoid Actions and Their Smooth Flows of Weights
}

\author{
By \\ Takehiko YAMANOUCHI*
}

\section{§0. Introduction}

In [Y1], we introduced notions of an action and a coaction of a measured groupoid on a von Neumann algebra, which are regarded as natural generalizations of group actions and group coactions in [N\&T]. We also exhibited constructions of their crossed products and dual (co)actions. Moreover, it was shown that we could extend a Nakagami-Takesaki duality theorem for group actions and coactions to the groupoid setting, although, in order to prove duality for coactions of measured groupoids, we needed to restrict ourselves to integrable ones. Several examples of groupoid (co)actions were also considered there. However, since our principal concern in that paper was to show the duality, some important things were left untouched. One of them is to locate the commutant of the crossed product by a groupoid action. To compute the commutant is, of course, very essential to analyze the structure of a crossed product, such as the factoriality and its algebraic type in the sense of Murray-von Neumann. The program in this direction was successfully accomplished in the case of group actions by realizing a crossed product as the left von Neumann algebra of a left Hilbert algebra. This realization enables us to know a modular operator and a modular conjugation of the crossed product. Our aim in this paper is to extend this technique to the groupoid setting. In the group action case, several mathematicians were engaged in the above program such as [T2], [D], [H2] and [S1]. Here we will closely follow works of Haagerup and Sauvageot in particular.

Now we describe the plan of this paper. In $\S 1$, we first review the P. Hahn's construction of a groupoid von Neumann algebra from a measured groupoid. His method is to construct a Tomita algebra, denoted by $\mathfrak{P}_{l}$, out of a given measured groupoid, and a groupoid von Neumann algebra is, by definition, the left von Neumann algebra associated with the Tomita algebra. We shall form a

Communicated by H. Araki, February 13, 1991

1991 Mathematics Subject Classification: 46L10, 46L55

* Department of Mathematics, University of California, Los Angeles, 405 Hilgard Ave., Los Angeles, CA 90024 U.S.A.; Current address: Department of Mathematics and Computer Science, University College of Swansea, Singleton Park, Swansea SA2 8PP United Kingdom 
new left Hilbert algebra $\mathfrak{B}$, from a measured groupoid, which is a subalgebra of $\mathfrak{P}_{l}$, but still equivalent to $\mathfrak{P}_{l}$. We make use of this new algebra in the next section. Section 2 is concerned with constructing a left Hilbert algebra from an action $\left(\mathscr{G},\{\mathcal{M}(x)\},\left\{\alpha_{\gamma}\right\}\right)$ of a measured groupoid $\mathscr{G}$ whose left von Neuman algebra coincides with the crossed product $\mu \times{ }_{\alpha} G$ by the action. One consequence of this is that $M \times{ }_{a} G$ is generated by operators of the two kinds, as in the case of group actions. Another consequence is that we can locate the commutant of the crossed product. In $\$ 3$, we compute relative commutants of some subalgebras of the crossed product by a principal measured groupoid. As a result, the center of the crossed product can be identified as the fixed point algebra of a certain groupoid action. In $\$ 4$, as one of the applications of the result in $\$ 3$, we compute the $T$-set of a crossed product. Section 5 is devoted to computation of the smooth flow of weights of the crossed product by a principal measured groupoid action. In $\S 6$, we give several examples of actions of principal groupoids. These examples will be constructed by exhibiting a homomorphism from a given groupoid into the normalizer of an (ergodic) countable group $G$ of automorphisms on a Lebesgue space $(\Omega, m)$. The homomorphism is lifted to the one into the group of automorphisms of the von Neumann algebra obtained by the group measure space construction from $(G, \Omega, m)$. Thus we get an action of the given groupoid. This construction was suggested to the author by Hamachi. We prove in Theorem 6.7 that the crossed product arising from such an action can be captured as a von Neumann algebra derived from a measured equivalence relation. Finally, we consider some concrete examples of actions of the type described above, one of which is due to Hamachi, and determine the algebraic types of their crossed products.

This work was done while the author stayed in Japan as a postdoctoral scholar at University of California, Los Angeles. The author heartily thanks Professor Masamichi Takesaki for having given him such an occasion.

The author would like to express his sincere gratitude to Professor Toshihiro Hamachi for helpful suggestion and fruitful discussion.

\section{$\S$ 耳. A Left Hilbert Algebra $\mathfrak{B}_{I}$}

In this section, we first establish several facts on a left Hilbert algebra derived from a measured groupoid, which will be used in the next section. Then we review the construction of a crossed product algebra from a given action of a measured groupoid.

Throughout this note, we fix a standard Borel groupoid $\mathscr{G}$. We assume that all relevant maps and sets that are related to the groupoid structure of $\mathscr{G}$ are Borel. We denote the source (resp. the range) of an element $\gamma$ of the groupoid by $s(\gamma)$ (resp. $r(\gamma)$ ). The unit space of $\mathscr{G}$, which is the image of the groupoid under the source (or the range) map, is denoted by $X$. For every $x \in X, \mathscr{G}^{x}$ 
(resp. $\mathscr{G}_{x}$ ) designates the inverse image of the range (resp. the source) map $r^{-1}(x)$ (resp. $s^{-1}(x)$ ). We assume from now on that the groupoid admits a faithful proper transverse function $\left\{\lambda^{x}\right\}_{x \in X}$ and a transverse measure $\Lambda$ with a module $\delta$. For these terminologies and basic properties of transverse functions and transverse measures, we refer readers to [C2]. Given such a system $\left(\left\{\lambda^{x}\right\}_{x \in X}, \Lambda, \delta\right)$ on $\mathscr{G}$, we have a $\sigma$-finite measure, denoted by $\Lambda_{\lambda}$ (or, simply by $\mu$ if there is no danger of confusion), on the unit space $X$. Let $v$ be the $\sigma$-finite measure on $\mathscr{G}$ given by integrating the transverse function with respect to the measure $\mu$. We also let $\lambda_{x}(x \in X)$ and $v^{-1}$ be the images of $\lambda^{x}$ and $v$, respectively, by the inverse map. By definition, $v$ is equivalent to $v^{-1}$ in the sense of absolute continuity and the module $\delta$ is the Radon-Nikodym derivative $d v / d v^{-1}$. Hereafter we call a groupoid with such a system a measured groupoid. Given a measured groupoid, we may construct a von Neumann algebra out of it, following Hahn's idea. The algebra is obtained as the left von Neumann algebra associated with a left Hilbert algebra $\mathfrak{I}_{I}$. In [Y1], we call the algebra a groupoid von Neumann algebra and denote it by $\mathscr{R}(\mathscr{G})$ without referring a system $\left(\left\{\lambda^{x}\right\}_{x \in X}, \Lambda, \delta\right)$. Now let us review the construction of the left Hilbert algebra : $\mathfrak{l}_{I}$. Define

$$
\mathfrak{P}_{I}=\left\{\xi \in L^{2}(\mathscr{G}, v): \xi \text { is } \delta \text {-bounded, }\|\xi\|_{I}<\infty\right\} .
$$

See [Ha2] for the definitions of $\delta$-boundedness and the norm $\|\cdot\|_{I}$. The definition of the set $\mathfrak{T}_{I}$ is exactly the same as that of $\bigcup_{I}$ in [Ha2]. However, it should be remarked that our situation is slightly different from $\mathbb{P}$. Hahn's in [Ha2] in that our measure $\mu$ may or may not be a probability one, while his $\mu$ is a probability measure. In any case, it can be shown as in [Ha2] that this set is an involutive (\#) algebra with the convolution as its product, where the convolution $*$ and the involution \# are defined respectively by

$$
\begin{aligned}
(f * g)\left(\gamma_{1}\right) & =\int f(\gamma) g\left(\gamma^{-1} \gamma_{1}\right) d \lambda^{r\left(\gamma_{1}\right)}(\gamma) \quad\left(f, g \in \mathfrak{P}_{l}\right), \\
f^{\#}(\gamma) & =\delta(\gamma)^{-1} \overline{f\left(\gamma^{-1}\right)} .
\end{aligned}
$$

Indeed, we can verify that, for any $\xi$ and $\eta$ in $: \mathfrak{T}_{I},\left\|\xi^{\#}\right\|_{I}=\|\xi\|_{I} ;\|\xi * \eta\|_{I} \leq$ $\|\xi\|_{I}\|\eta\|_{I} ;\left\|\xi^{\#}\right\|_{2} \leq \sqrt{a}\|\xi\|_{2}$ if $\xi$ is $\delta_{a}$-bounded; $\|\xi * \eta\|_{2} \leq\|\xi\|_{I}\|\eta\|_{2} ; \xi$ is $\delta_{a}$-bounded $\Leftrightarrow \xi^{\#}$ is $\delta_{a}$-bounded; if $\xi$ and $\eta$ are $\delta_{a}$ and $\delta_{b}$-bounded, respectively, then $\xi * \eta$ is $\delta_{a b}$-bounded.

Now we choose a symmetric probability measure $\tau$ from the measure class $[v]$. We put $\tilde{\tau}=r_{+}(\tau)$ (namely, $\tilde{\tau}(E)=\tau\left(r^{-1}(E)\right)$ ), which is a probability measure on $X$. We define two Borel functions $P$ and $Q$ on $\mathscr{G}$ by

$$
P(\gamma)=\frac{d v}{d \tau}(\gamma), \quad Q(\gamma)=\frac{d \tilde{\tau}}{d \mu} \circ r(\gamma) \quad(\gamma \in \mathscr{G}) .
$$

Let 


$$
\tau=\int \tau^{x} d \widetilde{\tau}(x), \quad v=\int v^{x} d \widetilde{\tau}(x)
$$

be the $r$-decompositions of $\tau$ and $v$ with respect to $\tau$, respectively. By Theorem 2.1 in [Ha1], almost all $\tau^{x}$ are probability measures and $P=d v^{x} / d \tau^{x}$ a.e..

Observation 1.1. Let $f$ be a nonnegative Borel function on $\mathscr{G}$. Then, for any Borel function $h$ on $X$, we have

$$
\begin{aligned}
\int\left(\int f(\gamma) d \lambda^{x}(\gamma)\right) h(x) d \mu(x) & =\int f(\gamma) h \circ r(\gamma) d v(\gamma) \\
& =\int\left(\int f(\gamma) d v^{x}(\gamma)\right) h(x) d \widetilde{\tau}(x) \\
& =\int\left(\int f(\gamma) Q(\gamma) d v^{x}(\gamma)\right) h(x) d \mu(x) .
\end{aligned}
$$

Since $h$ is arbitrary, it follows that

$$
\int f(\gamma) d \lambda^{x}(\gamma)=\int f(\gamma) Q(\gamma) d v^{x}(\gamma)
$$

for $\mu$ a.e. $x$ in $X$.

Observation 1.2. Let $f$ be an arbitrary Borel function on $\mathscr{G}$. Then we calculate

$$
\begin{aligned}
\int f(\gamma) \delta(\gamma)^{-1} d v(\gamma) & =\int f\left(\gamma^{-1}\right) \delta(\gamma) d v^{-1}(\gamma) \\
& =\int f\left(\gamma^{-1}\right) d v(\gamma) \\
& =\int f\left(\gamma^{-1}\right) P(\gamma) d \tau(\gamma) \\
& =\int f(\gamma) P\left(\gamma^{-1}\right) d \tau(\gamma) \\
& =\int f(\gamma) P\left(\gamma^{-1}\right) P(\gamma)^{-1} d v(\gamma)
\end{aligned}
$$

The fourth step is guaranteed by the assumption that $\tau$ is symmetric. From this calculation, we see that

$$
\delta(\gamma)=P(\gamma) P\left(\gamma^{-1}\right)^{-1}
$$

for $v$ a.e. $\gamma \in \mathscr{G}$.

For any $a \geq 1$, we set 


$$
E(a)=\left\{\gamma \in \mathscr{G}: P(\gamma), P\left(\gamma^{-1}\right), Q(\gamma) \in[1 / a, a]\right\},
$$

which is a Borel subset of $\mathscr{G}$. Before we state the following lemma that is essentially due to Hahn, we let $1_{E}$ denote the characteristic function of a set $E$.

Lemma 1.3. If $f=f 1_{E(a)}$ a.e. for some $a \geq 1$ and $f \in L^{\infty}(\mathscr{G}, v)$, then $f \in \mathfrak{P}_{I}$.

Proof. We put $M=\|f\|_{x}$. Then we have

$$
\begin{aligned}
\int|f(\gamma)|^{2} d v(\gamma) & =\int|f(\gamma)|^{2} P(\gamma) d \tau(\gamma) \\
& \leq M^{2} a<\infty
\end{aligned}
$$

because $\tau$ is a probability measure. So $f$ belongs to $L^{2}(\varphi, v)$. Next, due to Observation 1.1, we have that, for $\mu$ a.e. $x \in X$,

$$
\begin{aligned}
\int|f(\gamma)| d \lambda^{x}(\gamma) & =\int|f(\gamma)| Q(\gamma) d v^{x}(\gamma) \\
& =\int|f(\gamma)| Q(\gamma) P(\gamma) d \tau^{x}(\gamma) \\
& \leq M a^{2}<\infty,
\end{aligned}
$$

since almost all $\tau^{x}$ are probability measures. In view of Observation 1.1 and 1.2, we also have

$$
\begin{aligned}
\int\left|f^{\#}(\gamma)\right| d \lambda^{x}(\gamma) & =\int \delta(\gamma)^{-1}\left|f\left(\gamma^{-1}\right)\right| d \lambda^{x}(\gamma) \\
& =\int P(\gamma)^{-1} P\left(\gamma^{-1}\right)\left|f\left(\gamma^{-1}\right)\right| Q(\gamma) P(\gamma) d \tau^{x}(\gamma) \\
& \leq M a^{4}<\infty .
\end{aligned}
$$

Thus we conclude $\|f\|_{I}<\infty$. Finally we show that $f$ is $\delta$-bounded. Note first that the subset $\{\gamma \in \mathscr{G}: f(\gamma) \neq 0\} \backslash E(a)$ is a null set. It follows from Observation 1.2 that $\delta(\gamma)=P(\gamma) P\left(\gamma^{-1}\right)^{-1} \in\left[1 / a^{2}, a^{2}\right]$ for almost all $\gamma \in E(a)$ with $f(\gamma) \neq 0$. Therefore, $f$ belongs to ?I $_{I}$.

Q.E.D.

We have the following corollary to the above lemma, whose proof can be seen in Corollary 2.7 in [Ha2].

Corollary 1.4. The set $\mathfrak{T}_{I} \cap L^{x}(\mathscr{G}, v)$ is dense in $L^{2}(\mathscr{G}, v)$.

Lemma 1.5. (Lemma 2.9 in [Ha2]). If $h \in \mathfrak{P}_{I}$ is such that $(f * g \mid h)=0$ for all $f, g \in \mathfrak{P} I \cap L^{x}(G, v)$, then $h=0$ a.e..

Proof. Let $\{F(m)\}_{m \geq 1}$ be a countable generating family for the Borel 
structure of $\mathscr{G}$. Let $f_{n, m}=1_{E(n)} 1_{F(m)}$ and $f_{n, m}^{\prime}=f_{n, m} P^{-1}$. By Lemma 1.3, both $f_{n, m}$ and $f_{n, m}^{\prime}$ belong to $\mathfrak{A}_{I} \cap L^{\infty}(\mathscr{G}, v)$. By Fubini's theorem on a measured groupoid [Ha1], we have

$$
\begin{aligned}
0 & =\left(f_{n, \mathrm{~m}}^{\prime} * f_{p, q} \mid h\right) \\
& =\int\left(\int f_{p, q}\left(\gamma_{1}^{-1} \gamma\right) \overline{h(\gamma)} d \lambda^{r\left(\gamma_{1}\right)}(\gamma)\right) f_{n, m}\left(\gamma_{1}\right) d \tau\left(\gamma_{1}\right) .
\end{aligned}
$$

Since $h \in \mathfrak{N}_{I}, \iint f_{p, q}\left(\gamma_{1}^{-1} \gamma\right)|h(\gamma)| d \lambda^{r\left(\gamma_{1}\right)}(\gamma) d \tau\left(\gamma_{1}\right) \leq\|h\|_{I}$. By the dominated convergence theorem, we have that $\int_{F(m)} \int f_{p, q}\left(\gamma_{1}^{-1} \gamma\right) \overline{h(\gamma)} d \lambda^{r\left(\gamma_{1}\right)}(\gamma) d \tau\left(\gamma_{1}\right)=0$. Since $\{F(m)\}$ is a generating family, there exists a null Borel subset $N$ of $\mathscr{G}$ such that for each $\gamma_{1} \in G \backslash N, \int f_{p, q}\left(\gamma_{1}^{-1} \gamma\right) \overline{h(\gamma)} d \lambda^{r\left(\gamma_{1}\right)}(\gamma)=0$ for any pair $(p, q) \in$ $\mathbf{N} \times \mathbb{N}$. By the dominated convergence theorem, there is a null Borel set $N_{1}$ such that, if $\left(m, \gamma_{1}\right) \in \mathbb{N} \times\left(\mathscr{G} \backslash N_{1}\right)$, then $\int 1_{F(m)}\left(\gamma_{1}^{-1} \gamma\right) \overline{h(\gamma)} d \lambda^{r\left(\gamma_{1}\right)}(\gamma)=0$. Thus, if $\left(m, \gamma_{1}\right) \in \mathbb{N} \times\left(\mathscr{G} \backslash N_{1}\right)$, then we have $\int 1_{F(m)}(\gamma) \overline{h\left(\gamma_{1} \gamma\right)} d \lambda^{s\left(\gamma_{1}\right)}(\gamma)=0$. Hence, if $\gamma_{1} \in \mathscr{G} \backslash N_{1}$, then the function $\gamma \mapsto h\left(\gamma_{1} \gamma\right)$ is 0 for $\lambda^{s\left(\gamma_{1}\right)}$-a.e. $\gamma$, so that $\int\left|h\left(\gamma_{1} \gamma\right)\right|^{2} d \lambda^{s\left(\gamma_{1}\right)}(\gamma)=0$ for all $\gamma_{1} \in \mathscr{G} \backslash N_{1}$. Let us take a positive Borel function $f$ on $\mathscr{G}$ such that $\int f(\gamma) d \lambda^{x}(\gamma)=1$ for all $x \in X$. The existence of such a function $f$ is guaranteed by Lemma 3 in [C2]. Then, by Fubini's theorem, we have

$$
\begin{aligned}
0 & =\iint\left|h\left(\gamma_{1} \gamma\right)\right|^{2} d \lambda^{s\left(\gamma_{1}\right)}(\gamma) f\left(\gamma_{1}\right) d v\left(\gamma_{1}\right) \\
& =\iiint|h(\gamma)|^{2} d \lambda^{r\left(\gamma_{1}\right)}(\gamma) f\left(\gamma_{1}\right) d \lambda^{x}\left(\gamma_{1}\right) d \mu(x) \\
& =\iiint|h(\gamma)|^{2} f\left(\gamma_{1}\right) d \lambda^{r(\gamma)}\left(\gamma_{1}\right) d \lambda^{x}(\gamma) d \mu(x) \\
& =\int|h(\gamma)|^{2} d v(\gamma)
\end{aligned}
$$

Therefore $h=0$ a.e..

Q.E.D.

Lemma 1.6. The conjugate linear operator $S: \xi \mapsto \xi^{\#}$ defined on $\mathfrak{T}_{I}$ is preclosed.

Proof. First we note that $\eta^{b} \in \mathfrak{H}_{I}$ whenever $\eta$ is in $\mathfrak{P}_{I}$, where $g \triangleright{ }^{\triangleright}(\gamma)=\overline{g\left(\gamma^{-1}\right)}$. For any $\xi, \eta \in \mathfrak{P}_{l}$, we calculate

$$
\begin{aligned}
\left(\xi^{\#} \mid \eta\right) & =\int \delta(\gamma)^{-1} \overline{\xi\left(\gamma^{-1}\right) \eta(\gamma)} d v(\gamma) \\
& =\int \overline{\xi\left(\gamma^{-1}\right) \eta(\gamma)} d \widehat{v}(\gamma) \\
& =\int \eta^{\mathrm{b}}(\gamma) \overline{\xi(\gamma)} d v(\gamma) \\
& =\left(\eta^{\mathrm{b}} \mid \xi\right) .
\end{aligned}
$$


This shows that $\eta$ is in the domain of the adjoint of the \#-operation $S$ and that its image is $\eta^{b}$. Since $\mathfrak{T}_{I}$ is dense in $L^{2}(\mathscr{G}, v)$ by Lemma 1.3 , the adjoint is densely defined, so that \#-operation is preclosed.

Q.E.D.

It is now readily checked from the discussion so far that, letting $J f=\delta^{1 / 2} f^{\#}$ $\left(f \in L^{2}(\varphi, v)\right), \mathfrak{T}_{I}$ with multiplication *, left involution \#, is a left Hilbert algebra (in fact, a Tomita algebra) with the corresponding modular conjugation $J$, the right involution $b$ and the modular operator $\Delta$ given by

$$
\Delta \xi=\delta \xi \quad\left(\xi \in \mathfrak{P}_{l}\right) .
$$

Let $\mathscr{E}$ denote the abelian von Neumann algebra $L^{\infty}(X, \mu)$. On the Hilbert space $L^{2}(\mathscr{G}, v)$, we have two natural $\mathscr{L}$-actions; one is derived from a representation sending $h \in \mathscr{E}$ to an operator $M(h \circ r)$, and the other comes from a representation carrying $h \in \mathscr{Z}$ to $M(h \circ s)$, where $M(f)$ indicates the multiplication by $f \in L^{x}(\mathscr{G}, v)$. We call the former action the left action of $\mathscr{E}$ and the latter action the right action of $\mathscr{L}$. Thus $L^{2}(\mathscr{G}, v)$ becomes a $\mathscr{L}$-bimodule. The left (resp. right) action shall be written as $h \xi$ (resp. $\xi h)$, for any $h \in \mathscr{L}$ and $\xi \in$ $L^{2}(G, v)$. Set

$$
\mathscr{L}_{R}=\{M(h \circ r): h \in \mathscr{L}\}, \quad \mathscr{Z}_{s}=\{M(h \circ s): h \in \mathscr{L}\},
$$

which are both von Neumann subalgebras of $L^{x}(\mathscr{G}, v)$. From now on, we shall write ${ }_{\mathscr{L}} L^{2}(G, v)$ (resp. $\left.L^{2}(\mathscr{G}, v)_{\mathscr{L}}\right)$ when the left (resp. right) action is specifically considered on $L^{2}(\mathscr{G}, v)$. We define $D\left({ }_{n} L^{2}(\mathscr{G}, v), \mu\right)$ to be the set of all $\mu$ bounded vectors in $L^{2}(G, v)$ relative to the left action of $\mathscr{Z}$ with a faithful normal semifinite trace $\mu$, where the $\mu$-boundedness of a vector $\xi \in L^{2}(\mathscr{G}, v)$ is defined in [C3] as the property that there exists a positive number $C$ such that $\|h \xi\|^{2} \leq$ $C \int|h(x)|^{2} d \mu(x)$ for any $\mu$-square-integrable function $h \in \mathscr{L}$. We also define $D\left(L^{2}(\xi, v)_{\mathscr{\mathcal { F }}}, \mu\right)$ similarly for the right action. By Lemma 2.1 of [Y1], $\xi$ is in $D\left(x L^{2}(G, v), \mu\right)$ if and only if $\lambda\left(|\xi|^{2}\right) \in L^{x}(X, \mu)$, where $\lambda(f)$ is a function on $X$ definied by $\lambda(f)(x)=\int f(\gamma) d \lambda^{x}(\gamma)$ for any Borel function $f$ on $\mathscr{G}$. On the other hand, $\xi$ is in $D\left(L^{2}(\mathscr{G}, v)_{\mathcal{L}}, \mu\right)$ precisely when $\lambda^{\prime}\left(|\xi|^{2}\right) \in L^{\infty}(X, \mu)$, where $\lambda^{\prime}(f)$ is a function on $X$ given by $\lambda^{\prime}(f)(x)=\int f(\gamma) d \lambda_{x}^{\prime}(\gamma)$ with $d \lambda_{x}^{\prime}=\delta d \lambda_{r}$.

We now consider the following subspace of $\mathfrak{H}_{l}$ :

$$
\mathfrak{B}_{I}=\mathfrak{P}_{I} \cap D\left(\mathscr{L}_{\mathscr{L}} L^{2}(\mathscr{G}, v), \mu\right) \cap D\left(L^{2}(\mathscr{G}, v)_{\mathscr{E}}, \mu\right) .
$$

Our next purpose is then to show that $\mathfrak{B}_{I}$ becomes a self-adjoint subalgebra of the left Hilbert (Tomita) algebra $\mathfrak{P}_{I}$ and that $\mathfrak{B}_{I}$ is equivalent to $\mathfrak{V}_{I}$ as a left Hilbert algebra. Namely we will prove $\mathfrak{B}_{I}^{\prime \prime}=\mathfrak{Q} \mathfrak{Y}_{I}^{\prime \prime}$. In particular, they generate the same left von Neumann algebra $\mathscr{R}(\mathscr{G})$.

Let $\xi \in \mathfrak{B}_{I}$. We assume that $\xi$ is strictly $\delta_{a}$-bounded. Then we have

$$
\int\left|\xi^{\#}(\gamma)\right|^{2} d \lambda^{x}(\gamma)=\int \delta(\gamma)^{-2}\left|\xi\left(\gamma^{-1}\right)\right|^{2} d \lambda^{x}(\gamma)
$$




$$
\begin{aligned}
& =\int \delta(\gamma)^{2}|\xi(\gamma)|^{2} d \lambda_{x}(\gamma) \\
& =\int \delta(\gamma)|\xi(\gamma)|^{2} d \lambda_{x}^{\prime}(\gamma) \\
& \leq a\left\|\lambda^{\prime}\left(|\xi|^{2}\right)\right\|_{x} .
\end{aligned}
$$

This shows that $\xi \in D\left(\mathscr{L} L^{2}(\mathscr{G}, v), \mu\right)$. We also compute

$$
\begin{aligned}
\int\left|\xi^{\#}(\gamma)\right|^{2} d \lambda_{x}^{\prime}(\gamma) & =\int \delta(\gamma)^{-2}\left|\xi\left(\gamma^{-1}\right)\right|^{2} \delta(\gamma) d \lambda_{x}(\gamma) \\
& =\int \delta(\gamma)^{-1}\left|\xi\left(\gamma^{-1}\right)\right|^{2} d \lambda_{x}(\gamma) \\
& =\int \delta(\gamma)|\xi(\gamma)|^{2} d \lambda^{x}(\gamma) \\
& \leq a\left\|\lambda\left(|\xi|^{2}\right)\right\|_{x} .
\end{aligned}
$$

Thus $\xi$ belongs to $D\left(L^{2}(\mathscr{G}, v)_{\text {I }}, \mu\right)$. Accordingly the vector $\xi$ falls in $\mathfrak{B}_{I}$. It follows that $\mathfrak{B}_{I}$ is closed under \#-operation. Let $\eta$ be another vector in $\mathfrak{L}_{1}$. Then, by Schwarz inequality and Fubini's theorem,

$$
\begin{aligned}
\int|\xi * \eta(\gamma)|^{2} d \lambda^{x}(\gamma) & \leq \int\left(\int\left|\xi\left(\gamma_{1}\right)\right|\left|\eta\left(\gamma_{1}^{-1} \gamma\right)\right| d \lambda^{r(\gamma)}\left(\gamma_{1}\right)\right)^{2} d \lambda^{x}(\gamma) \\
& \leq \int\left(\int\left|\xi\left(\gamma_{1}\right)\right| d \lambda^{x}\left(\gamma_{1}\right)\right)\left(\int\left|\xi\left(\gamma_{1}\right)\right|\left|\eta\left(\gamma_{1}^{-1} \gamma\right)\right|^{2} d \lambda^{r(\gamma)}\left(\gamma_{1}\right)\right) d \lambda^{x}(\gamma) \\
& \leq\|\xi\|_{I} \int\left(\int\left|\xi\left(\gamma_{1}\right)\right|\left|\eta\left(\gamma_{1}^{-1} \gamma\right)\right|^{2} d \lambda^{r(\gamma)}\left(\gamma_{1}\right)\right) d \lambda^{x}(\gamma) \\
& =\|\xi\|_{I} \int\left(\int\left|\eta\left(\gamma_{1}^{-1} \gamma\right)\right|^{2} d \lambda^{r\left(\gamma_{1}\right)}(\gamma)\right)\left|\xi\left(\gamma_{1}\right)\right| d \lambda^{x}\left(\gamma_{1}\right) \\
& =\|\xi\|_{I} \int\left(\int|\eta(\gamma)|^{2} d \lambda^{s\left(\gamma_{1}\right)}(\gamma)\right)\left|\xi\left(\gamma_{1}\right)\right| d \lambda^{x}\left(\gamma_{1}\right) \\
& \leq\|\xi\|_{I}^{2}\left\|\lambda\left(|\eta|^{2}\right)\right\|_{\infty} .
\end{aligned}
$$

This means that $\xi * \eta$ lies in $D\left(\mathscr{L} L^{2}(\mathscr{G}, v), u\right)$. We also have

$$
\begin{aligned}
\int|\xi * \eta(\gamma)|^{2} d \lambda_{x}^{\prime}(\gamma) \leq & \int\left(\int\left|\xi\left(\gamma \gamma_{1}\right)\right|\left|\eta\left(\gamma_{1}^{-1}\right)\right| d \lambda^{s(\gamma)}\left(\gamma_{1}\right)\right)^{2} \delta(\gamma) d \lambda_{x}(\gamma) \\
\leq & \int\left(\int\left|\eta\left(\gamma_{1}^{-1}\right)\right| \delta\left(\gamma_{1}\right)^{-1} d \lambda^{s(\gamma)}\left(\gamma_{1}\right)\right) \\
& \left(\int \delta\left(\gamma_{1}\right)\left|\eta\left(\gamma_{1}^{-1}\right)\right|\left|\xi\left(\gamma \gamma_{1}\right)\right|^{2} d \lambda^{s(\gamma)}\left(\gamma_{1}\right)\right) \delta(\gamma) d \lambda_{x}(\gamma) \\
= & \int\left(\int\left|\eta^{\#}\left(\gamma_{1}\right)\right| d \lambda^{s(\gamma)}\left(\gamma_{1}\right)\right)
\end{aligned}
$$




$$
\begin{aligned}
& \left(\int \delta\left(\gamma_{1}\right)\left|\eta\left(\gamma_{1}^{-1}\right)\right|\left|\xi\left(\gamma \gamma_{1}\right)\right|^{2} d \lambda^{s(\gamma)}\left(\gamma_{1}\right)\right) \delta(\gamma) d \lambda_{x}(\gamma) \\
\leq & \|\eta\|_{I} \int\left(\int \delta\left(\gamma \gamma_{1}\right)\left|\xi\left(\gamma \gamma_{1}\right)\right|^{2} d \lambda_{r\left(\gamma_{1}\right)}(\gamma)\right)\left|\eta\left(\gamma_{1}^{-1}\right)\right| d \lambda^{x}\left(\gamma_{1}\right) \\
= & \|\eta\|_{I} \int\left(\int\|\xi(\gamma)\|^{2} d \lambda_{s\left(\gamma_{1}\right)}^{\prime}(\gamma)\right)\left|\eta\left(\gamma_{1}^{-1}\right)\right| d \lambda^{x}\left(\gamma_{1}\right) \\
\leq & \|\eta\|_{I}\left\|\lambda^{\prime}\left(|\xi|^{2}\right)\right\|_{\infty} \int\left|\eta\left(\gamma_{1}^{-1}\right)\right| d \lambda^{x}\left(\gamma_{1}\right) \\
\leq & a\|\eta\|_{I}^{2}\left\|\lambda^{\prime}\left(|\xi|^{2}\right)\right\|_{\infty},
\end{aligned}
$$

which implies that $\xi * \eta$ belongs to $D\left(L^{2}(\mathscr{G}, v)_{\mathscr{X}}, \mu\right)$. Therefore, we conclude that $\mathfrak{B}_{I}$ is a self-adjoint subalgebra of $\mathfrak{Y}_{I}$.

Lemma 1.7. The self-adjoint subalgebra $\mathfrak{B}_{I}$ is dense in $\mathfrak{P}_{I}$ with respect to the inner product $(\cdot \mid \cdot)_{\#}$ of $\mathfrak{H}_{\text {I }}$ given by the \#-operation. Namely, $(\xi \mid \eta)_{\#}=(\xi \mid \eta)+$ $\left(\xi^{\#} \mid \eta^{\#}\right)$ for any $\xi, \eta \in \mathfrak{P l}_{I}$.

Proof. First we note that $\mathfrak{L}_{I}$ is dense in $L^{2}(\mathscr{G}, v)$, because $\mathfrak{B}_{I}$ clearly contains the set $\mathfrak{T}_{I} \cap L^{\propto}(\mathscr{G}, v)$ which is already dense in $L^{2}(\mathscr{G}, v)$ by Corollary 1.4 .

Let $\eta \in \mathfrak{N}_{I}$ be such that $(\xi \mid \eta)_{\#}=0$ for all $\xi \in \mathfrak{B}_{I}$. This is equivalent to

$$
\int \xi(\gamma) \overline{\eta(\gamma)} d v(\gamma)+\int \overline{\xi(\gamma)} \eta(\gamma) \delta(\gamma) d v(\gamma)=0 .
$$

If we take $\xi$ to be a real-valued function, then we get

$$
\int \xi(\gamma)(\overline{\eta(\gamma)}+\delta(\gamma) \eta(\gamma)) d v(\gamma)=0
$$

Since $\mathfrak{B}_{I}$ is dense in $L^{2}(\mathscr{G}, v)$, we see that there exists a $v$-null subset $N$ of $\mathscr{G}$ such that $\overline{\eta(\gamma)}+\delta(\gamma) \eta(\gamma)=0$ for all $\gamma \in \mathcal{G} \backslash N$. It follows that, letting $\eta_{1}$ be the real part of $\eta$, we have that $(1+\delta(\gamma)) \eta_{1}(\gamma)=0$ for all $\gamma \in \mathscr{G} \backslash N$. Thus $\eta_{1}=0$ a.e.. Hence, if $\eta_{2}$ is the imaginary part of $\eta$, then equation (1) turns into

$$
\int \xi^{\prime}(\gamma) \eta_{2}(\gamma) d v(\gamma)=0
$$

where $\xi^{\prime}$ indicates the imaginary part of $\xi$. Since $\mathfrak{B}_{I}$ is dense in $L^{2}(\mathscr{G}, v), \eta_{2}=0$ a.e., which implies $\eta=0$ a.e.. Therefore, $\mathfrak{B}_{I}$ is dense in $\mathfrak{T}_{I}$ with respect to the inner product $(\cdot \mid \cdot)_{\#}$.

Q.E.D.

Proposition 1.8. The subalgebra $\mathfrak{B}_{I}$ is equivalent to $\mathfrak{H}_{I}$ as a left Hilbert algebra. Namely, we have $\mathfrak{B}_{I}^{\prime \prime}=\mathfrak{Q} \mathfrak{l}_{I}^{\prime \prime}$.

Proof. The assertion follows from the combination of Lemma 1.7 and 
For readers' convenience, we now briefly recall the construction of a crossed product algebra from an action of $\mathscr{G}$. A detailed discussion and various results concerning measured groupoid actions and crossed products by them are contained in [Y1], the notations of which we mainly adopt as well. Let ( $\mathscr{G}$, $\left.\{\mathcal{M}(x)\}_{x \in X},\left\{\alpha_{\gamma}\right\}_{\gamma \in \mathscr{G}}\right)$ be an action of our groupoid $\mathscr{G}$. Let $\mathscr{H}(x)$ be the canonical $L^{2}$-space of the von Neumann algebra $M(x)$ and $u(\gamma)$ be the canonical implementation of the *-isomorphism $\alpha_{\gamma}$ from $M(s(\gamma))$ onto $M(r(\gamma))$. Thus $u(\gamma)$ is the unique unitary from $\mathscr{H}(s(\gamma))$ onto $\mathscr{H}(r(\gamma))$ satisfying $\alpha_{\gamma}=\operatorname{Ad} u(\gamma)$. By definition, $\{M(x), \mathscr{H}(x)\}_{x \in X}$ is a measurable field of von Neumann algebras over $(X, \mu)$. Let $\{\mathcal{M}, \mathscr{H}\}$ be the von Neumann algebra obtained as the direct integral of the above field. Namely

$$
M=\int_{X}^{\oplus} \mu(x) d \mu(x), \quad \mathscr{H}=\int_{X}^{\oplus} \mathscr{H}(x) d \mu(x) .
$$

For each $x \in X$, we set $\widehat{\mathscr{H}}(x)=\mathscr{H}(x) \otimes L^{2}\left(\mathscr{G}^{x}, \lambda^{x}\right)$. Next we define a subspace $\widehat{M}(\gamma)$ of $\widehat{\mathscr{L}}(\mathscr{H}(s(\gamma)), \widehat{\mathscr{H}}(r(\gamma)))$ by

$$
\widehat{M}(\gamma)=\{a u(\gamma) \otimes \lambda(\gamma): a \in \mathcal{M}(r(\gamma))\},
$$

where $\lambda(\gamma)$ is a unitary operator from $L^{2}\left(\varphi^{s(\gamma)}, \lambda^{s(\gamma)}\right)$ onto $L^{2}\left(\varphi^{r(\gamma)}, \lambda^{r(\gamma)}\right)$ defined by

$$
\{\lambda(\gamma) g\}\left(\gamma_{1}\right)=g\left(\gamma^{-1} \gamma_{1}\right) \quad\left(g \in L^{2}\left(\varphi^{s(\gamma)}, \lambda^{s(\gamma)}\right), \gamma_{1} \in \varphi^{r(\gamma)}\right) .
$$

The symbol $\lambda$ is called the left regular representation of the measured groupoid $\mathscr{G}$. Since $\{\widehat{\mathscr{H}}(x)\}_{x \in X}$ is a measurable field of Hilbert spaces over $(X, \mu)$, we may form its direct integral:

$$
\int_{X}^{\oplus} \widehat{\mathscr{H}}(x) d \mu(x)=\int_{X}^{\oplus} \mathscr{H}(x) \otimes L^{2}\left(\mathscr{G}^{x}, \lambda^{r}\right) d \mu(x),
$$

which is equal to the relative tensor product $\mathscr{H} \otimes_{\mu \mathscr{L}} L^{2}(\mathscr{G}, v)$. Note that we can identify $\mathscr{H} \otimes_{\mu \mathscr{L}} L^{2}(\mathscr{G}, v)$ with the set of all functions $\eta$ from $\mathscr{G}$ into $\Pi_{x \in X} \mathscr{H}(x)$ such that (i) $\eta(\gamma) \in \mathscr{H}(r(\gamma)),(\gamma \in \mathscr{G})$. (ii) a function $x \in X \mapsto \int f_{m}(\gamma)\left(\xi_{n, x} \mid \eta(\gamma)\right)$ $d \lambda^{x}(\gamma)$ is measurable for any $m, n \in \mathbb{N}$, where $\left\{\xi_{n}\right\}_{n \geq 1}$ and $\left\{f_{m}\right\}_{m \geq 1}$ are fundamental sequences of measurable fields for $\{\mathscr{H}(x)\}$ and $\left\{L^{2}\left(\mathscr{G}^{x}, \lambda^{x}\right)\right\}$, respectively. (iii) $\int\|\eta(\gamma)\|^{2} d v(\gamma)<\infty$. The norm of such a function $\eta$ is defined by $\|\eta\|=$ $\left(\int\|\eta(\gamma)\|^{2} d v(\gamma)\right)^{1 / 2}$.

We let $\mathscr{S}\left(\mathscr{G}, \Pi_{\gamma \in \mathscr{G}} \widehat{M}(\gamma)\right)$ denote the set of all sections $A$ from $\mathscr{G}$ into $\Pi_{\gamma \in \mathscr{G}} \widehat{M}(\gamma)$ with the following properties:

(1) $\mathrm{A}$ is of the form $A(\gamma)=a(\gamma) u(\gamma) \otimes \lambda(\gamma)(a(\gamma) \in \mathcal{M}(r(\gamma)))$ for which a function $\gamma \in \mathscr{G}_{\mapsto} \mapsto<a(\gamma), \omega_{r(\gamma)}>$ is measurable for any $\omega=\int_{X}^{\oplus} \omega_{x} d \mu(x) \in \mathcal{M}_{+}=$ $\int_{X}^{\oplus} \mu(x)_{*} d \mu(x)$. 
(2) The quantity $\left.\|A\|_{H}=\max \quad\{\|\lambda(\|A(\cdot)\|)\|)\left\|_{\infty}, \quad\right\| \lambda\left(\left\|A^{\#}(\cdot)\right\|\right) \|_{\infty}\right\} \quad$ is bounded, where $A^{\#}(\gamma)=\delta(\gamma)^{-1} A\left(\gamma^{-1}\right)^{\prime}$. Note that, if $A(\cdot)$ is of the form $A(\gamma)=a(\gamma) u(\gamma) \otimes \lambda(\gamma)$ as before, then $A\left(\gamma^{-1}\right)^{+}$is given by $A\left(\gamma^{-1}\right)^{+}=$ $\alpha_{\gamma}\left(a\left(\gamma^{-1}\right)^{+}\right) u(\gamma) \otimes \lambda(\gamma)(\gamma \in G)$.

We will write $\mathscr{S}(\mathcal{M})=\mathscr{S}\left(\mathscr{G}, \Pi_{\gamma \in \mathscr{G}} \widehat{M}(\gamma)\right)$ for short, if there is no danger of confusion. $\mathscr{S}(\mathcal{M})$ becomes a vector space under pointwise addition and scalar multiplication. We can further equip $\mathscr{S}(\mathcal{M})$ with a \#-algebra structure. Its product $*$ and involution \# are given by

$$
\begin{aligned}
(A * B)(\gamma) & =\int A\left(\gamma_{1}\right) B\left(\gamma_{1}^{-1} \gamma\right) d \lambda^{r(\gamma)}\left(\gamma_{1}\right) \quad(A, B \in \mathscr{Y}(\mathcal{M})), \\
A^{\#}(\gamma) & =\delta(\gamma)^{-1} A\left(\gamma^{-1}\right)^{+} .
\end{aligned}
$$

See [Y1] for the details of this algebra. Next we shall define a representation of the algebra $\mathscr{S}(M)$ on a Hilbert space $\mathscr{H} \otimes_{\mu \mathscr{X}} L^{2}(\mathscr{G}, v)$. It will be obtained by "integrating" each section in $\mathscr{S}(M)$.

Let $A$ be in $\mathscr{S}(\mathcal{M})$ and $\xi, \eta \in \mathscr{H} \otimes_{\mu \mathscr{x}} L^{2}(\mathscr{G}, v)$. We may regard $\xi, \eta$ as functions on $\mathscr{G}$ as we observed before. The equation

$$
(\Phi(A) \xi \mid \eta)=\iint\left(a\left(\gamma_{1}\right) u\left(\gamma_{1}\right) \xi\left(\gamma_{1}^{-1} \gamma\right) \mid \eta(\gamma)\right) d \lambda^{r(\gamma)}\left(\gamma_{1}\right) d v(\gamma)
$$

defines a bounded operator $\Phi(A)$ on $\mathscr{H} \otimes_{\mu \nu} L^{2}(\mathscr{G}, v)$, where $A$ has the form $A(\gamma)=a(\gamma) u(\gamma) \otimes \lambda(\gamma), a(\gamma) \in \mathcal{M}(r(\gamma))$ for any $\gamma \in \mathscr{G}$. It turns out (see Lemma 4.2 of [Y1]) that $\Phi$ is a nondegenerate norm decreasing *-representation of the algebra $\mathscr{S}(\mathcal{M})$ on $\widehat{\mathscr{H}}=\mathscr{H} \otimes_{\mu \mathscr{x}} L^{2}(\mathscr{G}, v)$. The crossed product algebra of the action $\left(\mathscr{G},\{\mathcal{M}(x)\}_{x \in X},\left\{\alpha_{\gamma}\right\}_{\gamma \in \mathscr{G}}\right)$ of the groupoid $\mathscr{G}$ is by definition the weak closure of the nondegenerate $*$-algebra $\Phi(\mathscr{Y}(\mathcal{M}))$ and is denoted by $\mathcal{M} \times_{a} \mathscr{G}$. We showed in [Y1] that there exists a coaction of the groupoid on this new algebra which is called the dual coaction of the original action.

In the next section, we show another important way of constructing the crossed product algebra from an action, which enables us to locate the commutant of the algebra.

\section{§2. Another Construction of Crossed Products}

It is known that, in the case of a group action, its crossed product can be concretely realized as the left von Neumann algebra of a left Hilbert algebra (see [T2], [D], [H2] and [S1] for the construction of the left Hilbert algebra). One of the important applications of this realization may be that we can locate the commutant of the crossed product. Moreover it can be shown that there corresponds a canonical weight on the crossed product, called the dual weight, to each faithful normal semifinite weight on the algebra on which the group is acting. 
This section is devoted to following this line of thought in the groupoid setting. Namely, we are going to present a method of realizing the crossed product algebra of a groupoid action as the left von Neumann algebra of a left Hilbert algebra.

Let $\left(\mathscr{G},\left\{\lambda^{x}\right\}_{x \in X}, \Lambda, \delta\right)$ be a measured groupoid as before. We fix an action $\left(\mathscr{G},\{\mathcal{M}(x)\}_{x \in X},\left\{\alpha_{\gamma}\right\}_{\gamma \in \mathscr{G}}\right)$ of $\mathscr{G}$. We keep our notations from the last section. Assume the existence of a faithful normal state on $M$. Due to [Su], we may decompose it into $\varphi=\int_{X}^{\oplus} \varphi_{x} d \mu(x)$ along the direct integral $M=\int_{X}^{\oplus} \mu(x) d \mu(x)$. By Theorem 3.3 of [Su], every $\varphi_{x}$ is a faithful normal semifinite weight on $\mathcal{M}(x)$. Moreover, since $\varphi$ is a state, almost all $\varphi_{x}$ are finite. Thus, without any loss of generality, we may assume in the later discussion that each $\varphi_{x}$ is a faithful positive functional on $M(x)$. Let $\mathcal{H}_{\varphi_{1}}$ be the Hilbert space obtained by the GNS construction from $\varphi_{x}$. Since $\varphi_{x}$ is faithful, $\left\{M(x), \mathscr{H}_{\varphi_{1}}\right\}$ is a standard representation. So we may and do identify $\mathscr{H}_{\varphi}$, with the canonical $L^{2}$-space $\mathscr{H}(x)$ from now on.

For a notation, given a normal positive functional $\rho$ on a von Neumann algebra $\mathcal{N}$, we denote by $\eta_{\rho}$ the canonical injection of $\mathcal{N}$ into the Hilbert space $\mathscr{H}_{\rho}$ associated with the cyclic (GNS) representation $\left\{\pi_{\rho}, \mathscr{H}_{\rho}, \xi_{\rho}\right\}$ of $\mathcal{N}$.

Let $\mathscr{F}(M)$ be the set of functions $a$ from $\mathscr{G}$ into $\Pi_{x \in X} \mathcal{M}(x)$ with properties; (1) $a(\gamma) \in M(r(\gamma))(\gamma \in \mathscr{G})$ (2) for any pair $\xi=\int_{X}^{\oplus} \xi_{x} d \mu(x)$ and $\eta=\int_{X}^{\oplus} \eta_{x} d \mu(x)$ of elements in $\mathscr{H}=\int_{X}^{\oplus} \mathcal{H}(x) d \mu(x)$, a function $\gamma \in \mathscr{G}_{\mapsto} \mapsto\left(a(\gamma) \xi_{r(\gamma)} \mid \eta_{r(\gamma)}\right)$ is $v$ measurable. This set becomes a vector space under pointwise addition and scalar multiplication. Moreover we can introduce a \#-operation on $\mathscr{F}(M)$ by the formula; $a^{\#}(\gamma)=\delta(\gamma)^{-1} \alpha_{\gamma}\left(a\left(\gamma^{-1}\right)^{4}\right)(\gamma \in \mathscr{G}, a \in \mathscr{F}(M))$. Next we define a subset $\mathscr{F}_{\varphi}(M)$ of $\mathscr{F}_{F}(\mathcal{M})$ consisting of elements $a$ that satisfy the following conditions;

$$
\begin{aligned}
& \left\|f_{a}\right\|_{I} \ll \propto \text { and } \lambda\left(f_{a}^{2}\right), \lambda^{\prime}\left(f_{a}^{2}\right) \in L^{\infty}(X, \mu), \text { where } f_{a}(\gamma)=\|a(\gamma)\|(\gamma \in \mathscr{G}) . \\
& \int\left\|\eta_{\varphi_{s()^{\circ} \alpha^{-1}}}(a(\gamma))\right\|^{2} d v(\gamma)<\infty .
\end{aligned}
$$

We often write $\mathscr{F}_{\varphi}$ for $\mathscr{F}_{\varphi}(\mathcal{M})$ if there is no danger of confusion. It is easy to see that $\mathscr{F}_{\varphi}$ is a subspace of $\mathscr{F}_{\mathcal{F}}(\mathcal{M})$. For any pair $a, b$ of elements in $\mathscr{F}_{\varphi}$, we may consider their product $a * b$ given by the equation;

$$
(a * b)(\gamma)=\int a\left(\gamma_{1}\right) \alpha_{\gamma_{1}}\left(b\left(\gamma_{1}^{-1} \gamma\right)\right) d \lambda^{r(\gamma)}\left(\gamma_{1}\right) \quad(\gamma \in \mathscr{G})
$$

It can be readily verified that the product belongs to $\mathscr{F}(M)$. We shall show below that the product in fact falls in $\mathscr{F}_{\varphi}$. For this, we start off with the following lemma.

Lemma 2.1. Let $f$ and $g$ be nonnegative measurable functions on $\mathscr{G}$ such that $\|f\|_{I},\|g\|_{I}<\infty$ and functions $\lambda\left(f^{2}\right), \lambda\left(g^{2}\right), \lambda^{\prime}\left(f^{2}\right)$ and $\lambda^{\prime}\left(g^{2}\right)$ belong to $L^{\infty}(X, \mu)$. Then we have $\|f * g\|_{I}<\infty$, and both $\lambda\left((f * g)^{2}\right)$ and $\lambda^{\prime}\left((f * g)^{2}\right)$ are in $L^{\infty}(X, \mu)$. 
Proof. Since $\|f\|_{I},\|g\|_{I}<\infty$, it follows from [Ha2] that $\|f * g\|_{I}<\infty$.

Let $x \in X$. Then, by Schwarz inequality and Fubini's theorem, we have

$$
\begin{aligned}
\int\{(f * g)(\gamma)\}^{2} d \lambda^{x}(\gamma) & \leq \int\left(\int f\left(\gamma_{1}\right) g\left(\gamma_{1}^{-1} \gamma\right) d \lambda^{r(\gamma)}\left(\gamma_{1}\right)\right)^{2} d \lambda^{x}(\gamma) \\
& \leq \int\left(\int f\left(\gamma_{1}\right) d \lambda^{r(\gamma)}\left(\gamma_{1}\right)\right)\left(\int f\left(\gamma_{1}\right) g\left(\gamma_{1}^{-1} \gamma\right)^{2} d \lambda^{r(\gamma)}\left(\gamma_{1}\right)\right) d \lambda^{x}(\gamma) \\
& \leq\|f\|_{I} \int\left(\int g\left(\gamma_{1}^{-1} \gamma\right)^{2} d \lambda^{r\left(\gamma_{1}\right)}(\gamma)\right) f\left(\gamma_{1}\right) d \lambda^{x}\left(\gamma_{1}\right) \\
& =\|f\|_{I} \int\left(\int g(\gamma)^{2} d \lambda^{s\left(\gamma_{1}\right)}(\gamma)\right) f\left(\gamma_{1}\right) d \lambda^{x}\left(\gamma_{1}\right) \\
& \leq\|f\|_{I}^{2}\left\|\lambda\left(g^{2}\right)\right\|_{x} .
\end{aligned}
$$

Thus we have proven that $\lambda\left((f * g)^{2}\right) \in L^{\infty}(X, \mu)$. Moreover we compute

$$
\begin{aligned}
\int\{(f * g)(\gamma)\}^{2} \delta(\gamma) d \lambda_{x}(\gamma) \leq & \int\left(\int f\left(\gamma_{1}\right) g\left(\gamma_{1}^{-1} \gamma\right) d \lambda^{r(\gamma)}\left(\gamma_{1}\right)\right)^{2} \delta(\gamma) d \lambda_{x}(\gamma) \\
\leq & \int\left(\int f\left(\gamma_{1}\right) d \lambda^{r(\gamma)}\left(\gamma_{1}\right)\right) \\
& \left(\int f\left(\gamma_{1}\right) g\left(\gamma_{1}^{-1} \gamma\right)^{2} d \lambda^{r(\gamma)}\left(\gamma_{1}\right)\right) \delta(\gamma) d \lambda_{x}(\gamma) \\
\leq & \|f\|_{I} \iint f\left(\gamma_{1}\right) g\left(\gamma_{1}^{-1} \gamma\right)^{2} \delta(\gamma) d \lambda^{r(\gamma)}\left(\gamma_{1}\right) d \lambda_{x}(\gamma) \\
= & \|f\|_{I} \iint f\left(\gamma \gamma_{1}\right) g\left(\gamma_{1}^{-1}\right)^{2} \delta(\gamma) d \lambda^{s(\gamma)}\left(\gamma_{1}\right) d \lambda_{x}(\gamma) \\
= & \|f\|_{I} \iint f\left(\gamma \gamma_{1}\right) g\left(\gamma_{1}^{-1}\right)^{2} \delta(\gamma) d \lambda_{r\left(\gamma_{1}\right)}(\gamma) d \lambda^{x}\left(\gamma_{1}\right) \\
= & \|f\|_{I} \int\left(\int f\left(\gamma \gamma_{1}\right) \delta(\gamma) d \lambda_{r\left(\gamma_{1}\right)}(\gamma)\right) g\left(\gamma_{1}^{-1}\right)^{2} d \lambda^{x}\left(\gamma_{1}\right) \\
= & \|f\|_{I} \int\left(\int f^{\#}(\gamma) d \lambda^{s\left(\gamma_{1}\right)}(\gamma)\right) g\left(\gamma_{1}\right)^{2} \delta\left(\gamma_{1}\right) d \lambda_{x}\left(\gamma_{1}\right) \\
\leq & \|f\|_{I}^{2}\left\|\lambda^{\prime}\left(g^{2}\right)\right\|_{\infty} .
\end{aligned}
$$

This shows that $\lambda^{\prime}\left((f * g)^{2}\right)$ also belongs to $L^{x}(X, \mu)$.

Q.E.D.

Corollary 2.2. Let $a$ and $b$ be elements in $\mathscr{F}_{\varphi}$. Then we have $\left\|f_{a+b}\right\|_{I}<\infty$ and $\lambda\left(f_{a+b}^{2}\right), \lambda^{\prime}\left(f_{a+b}^{2}\right) \in L^{\curvearrowright}(X, \mu)$.

Proof. This is an easy consequence of combination of the inequality $f_{a+b} \leq$ $f_{a} * f_{b}$ and the previous lemma.

Q.E.D. 
It follows from the above corollary that, if $a, b \in \mathscr{F}_{\varphi}$, then $a * b$ satisfies condition (F1) for an element of $\mathscr{F}(\mathcal{M})$ to belong to $\mathscr{F}_{\varphi}$. Hence, in order to show that $a * b \in \mathscr{F}_{\varphi}$, we have to prove that the product also satisfies condition (F2). For this purpose, we need some preparation.

First we introduce a notation. Let $\rho$ and $\dot{\omega}$ be faithful normal semifinite weights on a von Neumann algebra $\mathcal{N}$. We consider semicyclic representations $\left\{\pi_{\rho}, \mathscr{H}_{\rho}\right\}$ and $\left\{\pi_{\omega}, \mathscr{H}_{\omega}\right\}$ of $\mathcal{N}$. It is known that these two representations are unitarily equivalent. We denote by $U_{\rho, \omega}$ the unique unitary from $\mathscr{H}_{\omega}$ onto $\mathscr{H}_{\rho}$ such that (i) $\pi_{\rho}(x)=U_{\rho, \omega} \pi_{\omega}(x) U_{\rho, \omega}^{+}(x \in \mathcal{N})$ (ii) $U_{\rho, \omega} \mathscr{P}_{\omega}^{\#}=\mathscr{P}_{\rho}^{\#}$, where $\mathscr{P}_{\omega}^{\#}$ (resp. $\mathscr{P}_{\rho}^{\#}$ ) is the self-dual positive cone in $\mathscr{H}_{\omega}$ (resp. $\mathscr{H}_{\rho}$ ) associated with $\omega$ (resp. $\rho$ ). Using this unitary, we are able to describe the unitary implementing the *isomorphism $\alpha_{\gamma}$. First consider a unitary $V(\gamma)$ defined by

$$
V(\gamma) \eta_{\varphi_{s(\gamma)}}(c)=\eta_{\varphi_{s(1)^{\circ}} \alpha_{\gamma^{\prime}}^{-1}}\left(\alpha_{\gamma}(c)\right) \quad(c \in \mathcal{M}(s(\gamma))) .
$$

$V(\gamma)$ carries $\mathscr{H}(s(\gamma))$ onto $\mathscr{H}_{\varphi_{s(1)}{ }^{\circ} \alpha_{\nu}^{-1}}$. Then $u(\gamma)$ is written as

$$
u(\gamma)=U_{\left.\varphi_{1(\gamma)}, \varphi_{1(1)}\right)^{-1}, \gamma} V(\gamma) .
$$

So we get

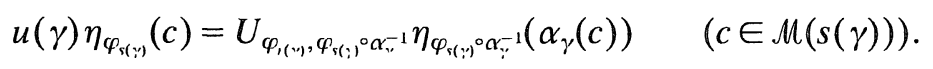

We will make good use of the above identity in the following discussion.

Now we define a linear map $\Lambda_{\varphi}$ from $\mathscr{F}(M)$ into the set of functions $\eta$ from $\mathscr{G}$ into $\Pi_{x \in X} \mathscr{H}(x)$ satisfying conditions (i) and (ii) that appeared in $\$ 1$ by

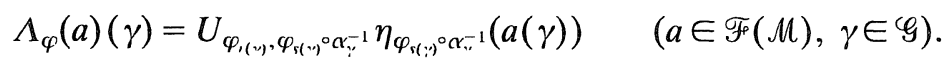

Note that $\Lambda_{\varphi}(a)$ satisfies condition (iii) in $\S 1$ as well, if $a$ belongs to $\mathscr{F}_{\varphi}$. Thus $\Lambda_{\varphi}(a)$ falls in $\mathscr{H}_{\mu \mathscr{L}} \mathcal{L}^{2}(\mathscr{G}, v)$ whenever $a \in \mathscr{F}_{\varphi}$.

Let $a$ and $b$ be in $\mathscr{F}_{\varphi}$. Then we compute

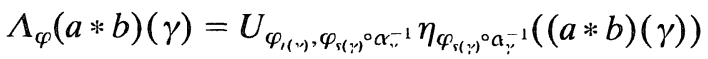

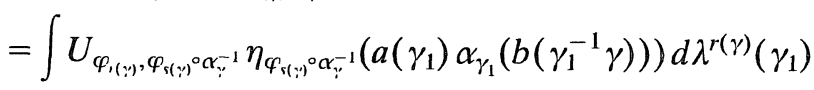

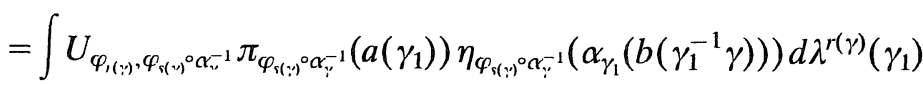

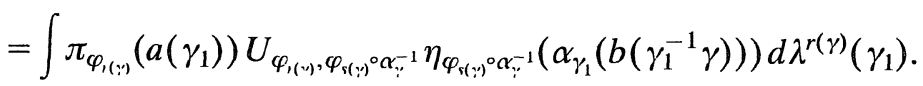

Due to the above identity (II), we calculate 


$$
\begin{aligned}
& U_{\varphi_{,(\nu, \gamma)}, \varphi_{s(\gamma)} \alpha^{\circ} \alpha_{\gamma}^{-1}} \eta_{\varphi_{s(\gamma)^{\circ}} \alpha_{\gamma}^{-1}}\left(\alpha_{\gamma_{1}}\left(b\left(\gamma_{1}^{-1} \gamma\right)\right)\right)=u(\gamma) \eta_{\varphi_{s(\gamma)}}\left(\alpha_{\gamma}^{-1} \alpha_{\gamma_{1}}\left(b\left(\gamma_{1}^{-1} \gamma\right)\right)\right) \\
& =u(\gamma) \eta_{\varphi_{\mathrm{s}(\gamma)}}\left(\alpha_{\gamma_{1}^{-1}}^{-1}\left(b\left(\gamma_{1}^{-1} \gamma\right)\right)\right) \\
& =u\left(\gamma_{1}\right) u\left(\gamma_{1}^{-1} \gamma\right) \eta_{\varphi_{s\left(v_{1}^{-1},\right)^{\prime}}}\left(\alpha_{\gamma_{1}^{-1} \gamma}^{-1}\left(b\left(\gamma_{1}^{-1} \gamma\right)\right)\right)
\end{aligned}
$$

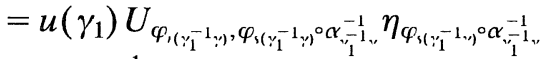

$$
\begin{aligned}
& \left(b\left(\gamma_{1}^{-1} \gamma\right)\right) \\
& =u\left(\gamma_{1}\right) \Lambda_{\varphi}(b)\left(\gamma_{1}^{-1} \gamma\right) \text {. }
\end{aligned}
$$

Thanks to this observation, we may continue the above computation of $\Lambda_{\varphi}(a * b)(\gamma)$ as follows;

$$
\Lambda_{\varphi}(a * b)(\gamma)=\int \pi_{\varphi_{,(*)}}\left(a\left(\gamma_{1}\right)\right) u\left(\gamma_{1}\right) \Lambda_{\varphi}(b)\left(\gamma_{1}^{-1} \gamma\right) d \lambda^{r(\gamma)}\left(\gamma_{1}\right) .
$$

Thus, if we define an element $A$ in $\mathscr{S}(\mathcal{M})$ by $A(\gamma)=a(\gamma) u(\gamma) \otimes \lambda(\gamma)$, then it follows that $\Lambda_{\varphi}(a * b)(\gamma)=\left\{\Phi(A) \Lambda_{\varphi}(b)\right\}(\gamma)$. Therefore we obtain the following proposition.

Proposition 2.3. Let $a$ and $b$ be elements of $\mathscr{F}_{\varphi}$. Then, defining an element $A$ in $\mathscr{S}(\mathcal{M})$ by the formula $A(\gamma)=a(\gamma) u(\gamma) \otimes \lambda(\gamma)$, we have

$$
\Lambda_{\varphi}(a * b)=\Phi(A) \Lambda_{\varphi}(b) .
$$

Hence $\Lambda_{\varphi}(a * b)$ defines an element in $\mathscr{H} \otimes_{\mu} L^{2}(\mathscr{G}, v)$.

Corollary 2.4. Let $a$ and $b$ be as above. Then $a * b$ belongs to $\mathscr{F}_{\varphi}$. Namely, $\mathscr{F}_{\varphi}$ is closed under the product operation $*$. Thus $\mathscr{F}_{\varphi}$ becomes an algebra.

Proof. As we noted before, it is enough to show that $a * b$ satisfies condi-

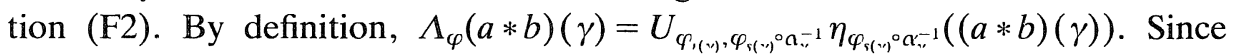
$U_{\varphi_{(, y)}, \varphi_{s(y))^{\circ} \alpha^{-1}}}$ is unitary, we have

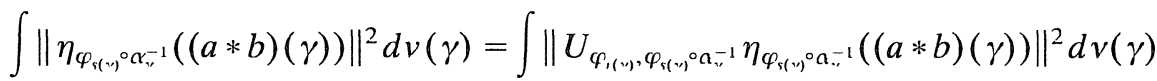

$$
\begin{aligned}
& =\int\left\|\Lambda_{\varphi}(a * b)(\gamma)\right\|^{2} d v(\gamma)<\infty .
\end{aligned}
$$

Therefore condition (F2) is satisfied.

Q.E.D.

Now we look at a \#-algebra $\mathscr{F}_{\varphi} \cap \mathscr{F}_{\varphi}^{\#}$. Let us then denote by $\widetilde{\mathfrak{P}}_{\varphi}$ the image of $\mathscr{F}_{\varphi} \cap \mathscr{F}_{\varphi}^{\#}$ in $\mathscr{H}_{\mu} \otimes_{\mu \mathscr{L}} L^{2}(\mathscr{G}, v)$ under the linear map $\Lambda_{\varphi}$. We will show next that $\mathscr{F}_{\varphi} \cap \mathscr{F}_{\varphi}^{\#}$, hence $\mathfrak{N}_{\varphi}$, contains plenty of elements which make $\widetilde{\mathfrak{T}}_{\varphi}$ dense in $\mathscr{H} \otimes_{\mu \mathscr{L}} L^{2}(\mathscr{G}, v)$.

Lemma 2.5. The set $\widetilde{\mathfrak{R}}_{\varphi}$ is a dense subspace of $\mathscr{H}_{\mu \mathscr{L}} \mathrm{L}^{2}(\mathscr{G}, v)$.

Proof. Let $b=\int_{X}^{\oplus} b(x) d \mu(x) \in \mathcal{M}=\int_{X}^{\oplus} \mathcal{M}(x) d \mu(x)$ and $h \in \mathfrak{B}_{I}$. Then we 
define an element $a_{b, h}$ in $\mathscr{F}(\mathcal{M})$ by $a_{b, h}(\gamma)=h(\gamma) b(r(\gamma))(\gamma \in \mathscr{G})$. Put $f_{a_{b, h}}(\gamma)=$ $\left\|a_{b, h}(\gamma)\right\|=\|h(\gamma) b(r(\gamma))\|=|h(\gamma)|\|b(r(\gamma))\|$. Note that $f_{a_{b, h}}(\gamma) \leq|h(\gamma)|\|b\|_{\infty}$. It follows that $\left\|f_{a_{b, h}}\right\|_{I}<\infty$ and $\lambda\left(f_{a_{b, h}}^{2}\right), \lambda^{\prime}\left(f_{a_{b, h}}^{2}\right) \in L^{\infty}(X, \mu)$. Moreover we have

$$
\begin{aligned}
\int\left\|\eta_{\varphi_{s(\gamma)^{\circ}} \alpha_{\gamma / 1}^{-1}}(a(\gamma))\right\|^{2} d v(\gamma) & =\int|h(\gamma)|^{2} \| \eta_{\varphi_{s(\gamma)}{ }^{\circ} \alpha_{\gamma}^{-1}}\left(b(r(\gamma)) \|^{2} d v(\gamma)\right. \\
& =\int|h(\gamma)|^{2} \varphi_{s(\gamma)}{ }^{\circ} \alpha_{\gamma}^{-1}\left(b(r(\gamma))^{+} b(r(\gamma))\right) d v(\gamma) \\
& \leq \int|h(\gamma)|^{2}\left\|\varphi_{s(\gamma)}\right\|\|b(r(\gamma))\|^{2} d v(\gamma) \\
& \leq\|b\|_{\infty}^{2} \iint|h(\gamma)|^{2}\left\|\varphi_{s(\gamma)}\right\| d \lambda_{x}^{\prime}(\gamma) d \mu(x) \\
& =\|b\|_{\infty}^{2} \int\left(\int|h(\gamma)|^{2} d \lambda_{x}^{\prime}(\gamma)\right)\left\|\varphi_{x}\right\| d \mu(x) \\
& \leq\|b\|_{\infty}^{2}\left\|\lambda^{\prime}\left(|h|^{2}\right)\right\|_{\infty} \int\left\|\varphi_{x}\right\| d \mu(x) \\
& =\|b\|_{\infty}^{2}\left\|\lambda^{\prime}\left(|h|^{2}\right)\right\|_{\infty}\|\varphi\|<\infty
\end{aligned}
$$

This proves that $a \in \mathscr{F}_{\varphi}$.

Let us now look at $a_{b, h}^{\#}$. Since $a_{b, h}^{\#}(\gamma)=\delta(\gamma)^{-1} \alpha_{\gamma}\left(a_{b, h}\left(\gamma^{-1}\right)^{+}\right)=$ $h^{\#}(\gamma) \alpha_{\gamma}(b(s(\gamma))), \quad f_{a_{b, h}^{*}}(\gamma)=\left|h^{\#}(\gamma)\right|\|b(s(\gamma))\|$. Thus we have $f_{a_{b, h}^{*}}(\gamma) \leq$ $\left|h^{\#}(\gamma)\right|\|b\|_{\infty}$, which implies that $\left\|f_{a_{b h}^{*}{ }_{h}}\right\|_{I}<\propto$ and $\lambda\left(f_{a_{b, h}^{*}}^{2}\right), \lambda^{\prime}\left(f_{a_{b h}^{*},}^{2}\right) \in L^{\infty}(X, \mu)$.

Furthermore we calculate

$$
\begin{aligned}
\int \| \eta_{\varphi_{s(Y)^{\circ} \mathrm{a}^{-1}}\left(a_{b, h}^{\#}(\gamma)\right) \|^{2} d v(\gamma)} & =\int \varphi_{s(\gamma)^{\circ}} \alpha_{\gamma}^{-1}\left(\left|h^{\#}(\gamma)\right|^{2} \alpha_{\gamma}\left(b(s(\gamma))^{+} b(s(\gamma))\right) d v(\gamma)\right. \\
& =\int\left|h^{\#}(\gamma)\right|^{2} \varphi_{s(\gamma)}\left(b(s(\gamma))^{+} b(s(\gamma))\right) d v(\gamma) \\
& =\int\left(\int\left|h^{\#}(\gamma)\right|^{2} d \lambda_{x}^{\prime}(\gamma)\right) \varphi_{x}\left(b(x)^{+} b(x)\right) d \mu(x) \\
& \leq\left\|\lambda^{\prime}\left(\left|h^{\#}\right|^{2}\right)\right\|_{\infty} \int \varphi_{x}\left(b(x)^{+} b(x)\right) d \mu(x) \\
& =\left\|\lambda^{\prime}\left(\left|h^{\#}\right|^{2}\right)\right\|_{\infty} \varphi\left(b^{+} b\right)<\infty .
\end{aligned}
$$

Hence $a_{b, h}^{\#}$ also belongs to $\mathscr{F}_{\varphi}$. Therefore $a_{b, h} \in \mathscr{F}_{\varphi} \cap \mathscr{F}_{\varphi}^{\#}$.

Next we consider the image of $a_{b, h}$ under the map $\Lambda_{\varphi}$. We have

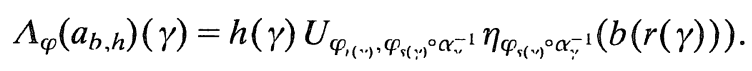

Let $\left\{c_{n}\right\}_{n \geq 1}$ be a countable family of measurable fields of operators such that $\left\{c_{n}(x)\right\}_{n \geq 1}$ generates $M(x)$ for almost every $x \in X$. Then, since $\mathfrak{R}_{I}$ is dense in $L^{2}(G, v)$, it is not so difficult to see that the subspace in $\mathscr{H} \otimes_{\mu \mathscr{L}} L^{2}(\mathscr{G}, v)$ generated by $\left\{\Lambda_{\varphi}\left(a_{c_{n}, h}\right): n \geq 1, h \in \mathfrak{B}_{I}\right\}$, which is contained in $\widetilde{\mathfrak{T}}_{\varphi}$, is dense in $\mathscr{H} \otimes_{\mu \mathscr{L}} L^{2}(\mathscr{G}, v)$.

Q.E.D. 
Our next aim is to show that the set $\widetilde{\mathfrak{F}}_{\varphi}$ becomes a left Hilbert algebra when it inherits the \#-algebra structure of $\mathscr{F}_{\varphi} \cap \mathscr{F}_{\varphi}^{\#}$ through the map $\Lambda_{\varphi}$. We shall also prove that the left von Neumann algebra of $\widetilde{\mathfrak{P}}_{\varphi}$ coincides with the crossed product algebra $\mathcal{M} \times{ }_{\alpha} \mathscr{G}$ by the action of $\mathscr{G}$. For this aim, we first introduce a nonsingular positive self-adjoit operator and a unitary conjugation on $\mathscr{H} \otimes_{\mu \mathscr{L}} L^{2}(\mathscr{G}, v)$, which turn out to be the modular operator and the modular conjugation of $\widetilde{\mathfrak{T}}_{\varphi}$.

In the following lemma, $\Delta_{\omega, \rho}$ denotes the relative modular operator corresponding to two faithful normal semifinite weights $\omega$ and $\rho$ on a von Neumann algebra.

Lemma 2.6. There exist a nonsingular positive self-adjoit operator $\widetilde{\Delta}_{\varphi}$ and a unitary conjugation $\widetilde{J}_{\varphi}$ on $\mathscr{H} \otimes_{\mu} L^{2}(\varphi, v)$ given by

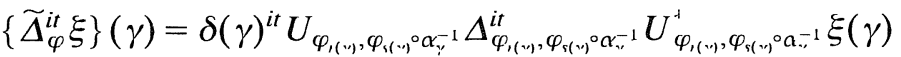

$$
\begin{aligned}
& \left\{\widetilde{J}_{\varphi} \xi\right\}(\gamma)=\delta(\gamma)^{-1 / 2} J_{\varphi,(\gamma)} u(\gamma) \xi\left(\gamma^{-1}\right) \quad\left(\xi \in \mathscr{H} \otimes_{\mu q} L^{2}(\mathscr{G}, v)\right) \text {, }
\end{aligned}
$$

where $J_{\varphi_{1}}$ is the unitary conjugation on $\mathscr{H}(x)$ associated with the standard representation $\{\mathcal{M}(x), \mathcal{H}(x)\}$.

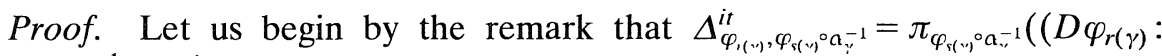
$\left.\left.D \varphi_{s(\gamma)} \circ \alpha_{\gamma^{\prime}}^{-1}\right) t\right) \Delta_{\varphi_{s(\nu)}{ }^{\circ} \alpha_{\nu}^{-1}}^{i t}$. Hence it is clear that the equation

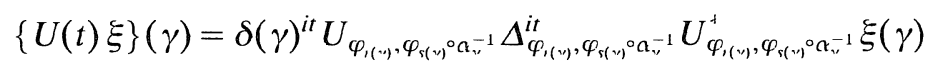

defines a one-parameter unitary group $\{U(t)\}$ on $\mathscr{H} \otimes_{\mu} L^{2}(\mathscr{G}, v)$. For continuity, we take vectors $\xi, \eta \in \mathscr{H} \otimes_{\mu \mathscr{L}} L^{2}(\mathscr{G}, v)$ and compute

$$
\begin{aligned}
& \lim _{t \rightarrow 0}(U(t) \xi \mid \eta)=\lim _{t \rightarrow 0} \int(\{U(t) \xi\}(\gamma) \mid \eta(\gamma)) d v(\gamma)
\end{aligned}
$$

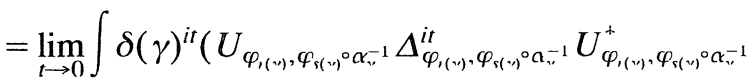

$$
\begin{aligned}
& \xi(\gamma) \mid \eta(\gamma)) d v(\gamma) \\
& =\int(\xi(\gamma) \mid \eta(\gamma)) d v(\gamma)=(\xi \mid \eta) \text {. }
\end{aligned}
$$

The third step is guarateed by Lebesgue dominated convergence theorem. This computation implies the continuity of $U(t)$. It follows from Stone's theorem that there exists a nonsingular positive self-adjoint operator $\widetilde{\Delta}_{\varphi}$ on $\mathscr{H} \otimes_{\mu \mathscr{L}} L^{2}(\mathscr{G}, v)$ such that

$$
U(t)=\widetilde{\Delta}_{\varphi}^{i t} \quad(t \in \mathbf{R}) .
$$

It is immediate to see that $\widetilde{J}_{\varphi}$ defined above is a conjugate linear unitary operator on $\mathscr{H} \otimes_{\mu} L^{2}(\mathscr{G}, v)$. The identity $\widetilde{J}_{\varphi}^{2}=1$ follows from the equation $J_{\varphi_{1(\nu)}} u(\gamma)=u(\gamma) J_{\varphi_{((N)}}(\gamma \in \mathscr{G})$.

Q.E.D. 
Lemma 2.7. The set $\widetilde{\mathfrak{T}}_{\varphi}$ is invariant under $\widetilde{\Delta}_{\varphi}^{i t}$ for any $t \in \mathbf{R}$. In fact, we have

$$
\widetilde{\Delta}_{\varphi}^{i t} \Lambda_{\varphi}(a)=\Lambda_{\varphi}\left(\rho_{t}^{\varphi}(a)\right) \quad\left(a \in \mathscr{F}_{\varphi} \cap \mathscr{F}_{\varphi}^{\#}\right),
$$

where $\rho_{t}^{\varphi}(a)(\gamma)=\delta(\gamma)^{i t}\left(D \varphi_{r(\gamma)}: D \varphi_{s(\gamma)} \circ \alpha_{\gamma}^{-1}\right)_{t} \sigma_{t}^{\varphi_{s(\gamma)} \circ \alpha_{\gamma}^{-1}}(a(\gamma))$.

Proof. First we will show that $\rho_{f}^{\varphi}(a) \in \mathscr{F}_{\varphi} \cap \mathscr{F}_{\varphi}^{\#}$ whenever $a \in \mathscr{F}_{\varphi} \cap \mathscr{F}_{\varphi}^{\#}$. Let $f_{\rho^{y}(a)}(\gamma)=\left\|\rho_{t}^{\varphi}(a)(\gamma)\right\|$. Then it is clear that we obtain

$$
f_{\rho_{t}^{\prime \prime}(a)}(\gamma) \leq\|a(\gamma)\|=f_{a}(\gamma) .
$$

Namely, $f_{\rho_{I}^{\prime}(a)} \leq f_{a}$. This implies that $\left\|f_{\rho_{I}^{\prime}(a)}\right\|_{I} \leq\left\|f_{a}\right\|_{I}<\infty$ and that $\lambda\left(f_{\rho_{I}^{\prime \prime}(a)}^{2}\right)$, $\lambda^{\prime}\left(f_{\rho_{l}^{\prime \prime}(a)}^{2}\right) \in L^{\propto}(X, \mu)$. Moreover we compute

$$
\begin{aligned}
\varphi_{s(\gamma)} \circ \alpha_{\gamma}^{-1}\left(\rho_{t}^{\varphi}(a)(\gamma)^{\dagger} \rho_{t}^{\varphi}(a)(\gamma)\right) & =\varphi_{s(\gamma)} \circ \alpha_{\gamma}^{-1}\left(\sigma_{t}^{\left.\varphi_{(\gamma ;}\right)^{\circ} \alpha_{\gamma}^{-1}}\left(a(\gamma)^{+} a(\gamma)\right)\right) \\
& =\varphi_{s(\gamma)} \circ \alpha_{\gamma}^{-1}\left(a(\gamma)^{\dagger} a(\gamma)\right) .
\end{aligned}
$$

It follows from this computation that

$$
\int\left\|\eta_{\varphi_{s(\gamma)^{\circ}} \alpha_{\gamma}^{-1}}\left(\rho_{t}^{\varphi}(a)(\gamma)\right)\right\|^{2} d v(\gamma)=\int\left\|\eta_{\varphi_{s(\gamma)^{\circ}} \alpha_{\gamma}^{-1}}(a(\gamma))\right\|^{2} d v(\gamma)<\infty .
$$

This shows that $\rho_{t}^{\varphi}(a) \in \mathscr{F}_{\varphi}$.

Next we look at $\rho_{t}^{\varphi}(a)^{\#}$. We have

$$
\rho_{t}^{\varphi}(a)^{\#}(\gamma)=\delta(\gamma)^{-1} \alpha_{\gamma}\left(\delta(\gamma)^{i t} \sigma_{t}^{\varphi_{1}(: ;)^{\circ} \alpha_{\gamma}^{-1}}\left(a\left(\gamma^{-1}\right)^{+}\right)\left(D \varphi_{s(\gamma)}: D \varphi_{r(\gamma)^{\circ}} \alpha_{\gamma}\right)_{t}^{+}\right) .
$$

So we have $f_{\rho_{t}^{\prime}(a)^{*}}(\gamma) \leq \delta(\gamma)^{-1}\left\|a\left(\gamma^{-1}\right)\right\|=f_{a^{*}}(\gamma)$. Namely $f_{\rho_{t}^{q}(a)^{*}} \leq f_{a^{*}}$. It follows that $\left\|f_{\rho_{I}^{q}(a)^{*}}\right\|_{I} \leq\left\|f_{a^{*}}\right\|_{I}<\infty$ and that $\lambda\left(f_{\rho_{l}^{q}(a)^{*}}^{2}\right), \lambda^{\prime}\left(f_{\rho_{I}^{q}(a)^{*}}^{2}\right) \in L^{\infty}(X, \mu)$.

Furthermore we compute

$$
\begin{aligned}
\varphi_{s(\gamma)^{\circ}} \alpha_{\gamma}^{-1}\left(\rho_{t}^{\varphi}(a)^{\#}(\gamma)^{+} \rho_{t}^{\varphi}(a)^{\#}(\gamma)\right) & =\delta(\gamma)^{-2} \varphi\left(\sigma_{t}^{\varphi_{s(i)}}\left(a\left(\gamma^{-1}\right) a\left(\gamma^{-1}\right)^{+}\right)\right) \\
& =\delta(\gamma)^{-2} \varphi_{s(\gamma)}\left(a\left(\gamma^{-1}\right) a\left(\gamma^{-1}\right)^{+}\right) \\
& =\varphi_{s(\gamma)}\left(a^{\#}(\gamma)^{+} a^{\#}(\gamma)\right) .
\end{aligned}
$$

Due to this calculation, we get

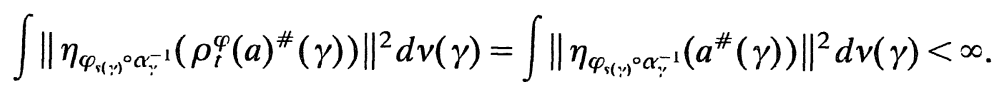

This means that $\rho_{t}^{\varphi}(a)^{\#} \in \mathscr{F}_{\varphi}$. So we conclude that $\rho_{t}^{\varphi}(a) \in \mathscr{F}_{\varphi} \cap \mathscr{F}_{\varphi}^{\#}$.

Finally we compute

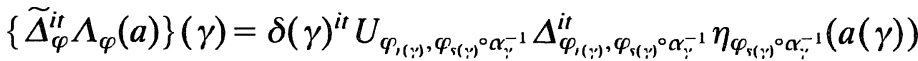

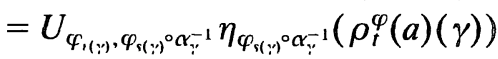

$$
\begin{aligned}
& =\Lambda_{\varphi}\left(\rho_{t}^{\varphi}(a)\right)(\gamma) \text {. }
\end{aligned}
$$


Therefore our assertion follows.

Q.E.D.

Lemma 2.8. We have the following identity;

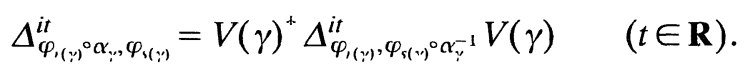

Therefore it yields

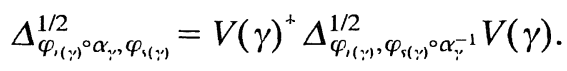

Proof. From the definition of $V(\gamma)$, it follows that

$$
\begin{aligned}
& V(\gamma) \Delta_{\left.\varphi_{s(\gamma)}\right)}^{i t}=\Delta_{\varphi_{s(:))^{\circ} \alpha^{-1}}^{i t}}^{i t} V(\gamma) \quad(t \in \mathbf{R})
\end{aligned}
$$

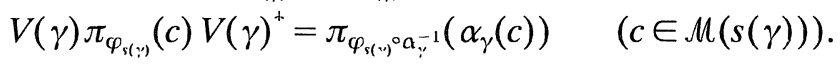

Due to these identities, we have

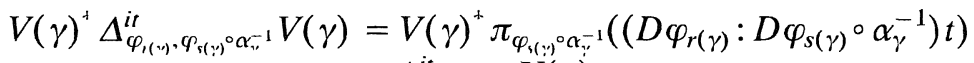

$$
\begin{aligned}
& \Delta_{\left.\varphi_{s(\gamma)}\right)^{\circ} a_{\gamma}^{-1}}^{i t} V(\gamma) \\
& =\pi_{\varphi_{s(;)}}\left(\alpha_{\gamma}^{-1}\left(\left(D \varphi_{r(\gamma)}: D \varphi_{s(\gamma)} \circ \alpha_{\gamma}^{-1}\right) t\right)\right) \Delta_{\varphi_{s(i)}}^{u t}
\end{aligned}
$$

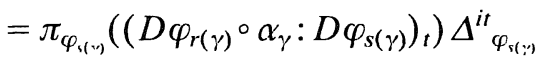

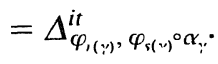

The second assertion is an easy consequence of the first one.

Q.E.D.

Lemma 2.9. The map $\Lambda_{\varphi}(a) \mapsto \Lambda_{\varphi}\left(a^{\#}\right)\left(a \in \mathscr{F}_{\varphi} \cap \mathscr{F}_{\varphi}^{\#}\right)$ is a densely defined preclosed linear operator on $\mathscr{H} \otimes_{\mu} L^{2}(\mathscr{G}, v)$ with its domain as $\widetilde{\mathfrak{T}}_{\varphi}$. It closure $\widetilde{S}_{\varphi}$ has a polar decomposition

$$
\widetilde{S}_{\varphi}=\widetilde{J}_{\varphi} \widetilde{\Delta}_{\varphi}^{1 / 2} .
$$

Proof. Let $a \in \mathscr{F}_{\varphi} \cap \mathscr{F}_{\varphi}^{\#}$. We compute

$$
\begin{aligned}
& \left\{\widetilde{J}_{\varphi} \Lambda_{\varphi}\left(a^{\#}\right)\right\}(\gamma)=\delta(\gamma)^{-1 / 2} J_{\varphi_{1(:)}} u(\gamma)\left\{\Lambda_{\varphi}\left(a^{\#}\right)\right\}\left(\gamma^{-1}\right)
\end{aligned}
$$

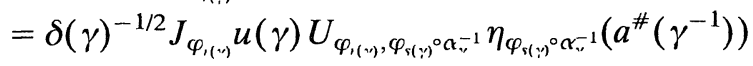

$$
\begin{aligned}
& =\delta(\gamma)^{1 / 2} u(\gamma) J_{\varphi_{\mathrm{s}(\gamma)}} U_{\varphi_{1(\gamma)}, \varphi_{\mathrm{s}(\gamma)^{\circ}} \alpha_{\gamma / \gamma}^{-1}} \eta_{\varphi_{\mathrm{s}(\gamma)^{\circ}} \alpha^{-1}}\left(\alpha_{\gamma}^{-1}(a(\gamma))^{+}\right)
\end{aligned}
$$

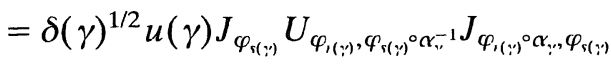

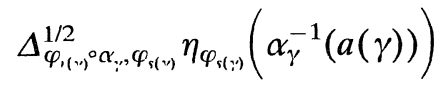

$$
\begin{aligned}
& =\delta(\gamma)^{1 / 2} u(\gamma) \Delta_{\varphi_{1(\gamma)}{ }^{\circ} \alpha_{\gamma,} \varphi_{s(\gamma)}}^{1 / 2} \eta_{\varphi_{\text {( }(:)}}\left(\alpha_{\gamma}^{-1}(a(\gamma))\right)
\end{aligned}
$$

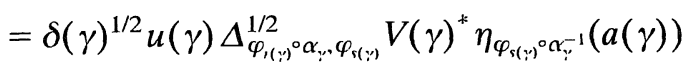

$$
\begin{aligned}
& =\delta(\gamma)^{1 / 2} u(\gamma) \Delta_{\varphi_{1(\gamma)}{ }^{\circ} \alpha_{, y,} \varphi_{s(\gamma)}}^{1 / 2} u(\gamma)^{+} \Lambda_{\varphi}(a)(\gamma)
\end{aligned}
$$

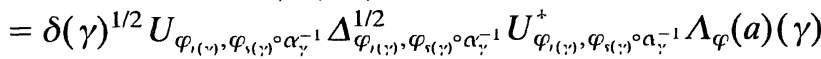




$$
=\left\{\widetilde{\Delta}_{\varphi}^{1 / 2} \Lambda_{\varphi}(a)\right\}(\gamma) .
$$

Here $J_{\rho, \omega}$ denotes the relative modular conjugation corresponding to two faithful normal semifinite weights $\rho$ and $\omega$ on a von Neumann algebra. The fifth step in the above calculation is guaranteed by the identity $U_{\varphi_{(\gamma, y}, \varphi_{s(y)}{ }^{\circ} \alpha_{y}^{-1}}=$ $J_{\left.\varphi_{,(\gamma)}\right)} J_{\varphi_{,(y)}, \varphi_{s(y)}{ }^{\circ} \alpha_{\gamma}^{-1}}$. The second last step is due to the previous lemma. Thanks to the computation, we have.

$$
\widetilde{J}_{\varphi} \Lambda_{\varphi}\left(a^{\#}\right)=\widetilde{\Delta}_{\varphi}^{1 / 2} \Lambda_{\varphi}(a)
$$

Namely

$$
\widetilde{J}_{\varphi} \widetilde{\Delta}_{\varphi}^{1 / 2} \Lambda_{\varphi}(a)=\Lambda_{\varphi}\left(a^{\#}\right)
$$

It follows that the map in question is a preclosed operator contained in $\widetilde{J}_{\varphi} \widetilde{\Delta}_{\varphi}^{1 / 2}$. For the second assertion, it is sufficient to show that $\widetilde{\mathfrak{T}}_{\varphi}$ is a core for $\widetilde{\Delta}_{\varphi}^{1 / 2}$. Let $p$ be the projection of $\mathcal{H}_{\mu \mathscr{L}} \mathcal{L}^{2}(\mathscr{G}, v)$ onto the closed subspace $\left[\left(1+\widetilde{\Delta}_{\varphi}^{1 / 2}\right) \widetilde{\mathfrak{A}}_{\varphi}\right]^{\perp}$. By Lemma $2.7, p$ commutes with $\Delta_{\varphi}$, so that

$$
p\left(1+\widetilde{\Delta}_{\varphi}^{1 / 2}\right) \subseteq\left(1+\widetilde{\Delta}_{\varphi}^{1 / 2}\right) p .
$$

Hence, if $\xi \in \widetilde{\mathfrak{P}}_{\varphi}$, we have

$$
\left(1+\widetilde{\Delta}_{\varphi}^{1 / 2}\right) p \xi=p\left(1+\widetilde{\Delta}_{\varphi}^{1 / 2}\right) \xi=0
$$

which implies that $p \xi=0$. Since $\widetilde{\mathfrak{N}}_{\varphi}$ is dense by Lemma 2.5 , it follows that $p=0$. Therefore $\widetilde{\mathfrak{N}}_{\varphi}$ is a core for $\widetilde{\Delta}_{\varphi}^{1 / 2}$.

Q.E.D.

As we saw just before Proposition 2.3, every element $a \in \mathscr{F}_{\varphi}$ gives rise to a member $A_{a}$ of $\mathscr{S}(\mathcal{M})$ given by $A_{a}(\gamma)=a(\gamma) u(\gamma) \otimes \lambda(\gamma)$. Remark that we have

$$
\begin{aligned}
A_{a * b} & =A_{a} * A_{b} \quad\left(a, b \in \mathscr{F}_{\varphi} \cap \mathscr{F}_{\varphi}^{\#}\right) \\
A_{a^{*}} & =A_{a}^{\#} .
\end{aligned}
$$

Thus the \#-algebraic structure of $\mathscr{F}_{\varphi} \cap \mathscr{F}_{\varphi}^{\#}$ is compatible with that of $\mathscr{S}(\mathcal{M})$ through the mapping $a \in \mathscr{F}_{\varphi} \cap \mathscr{F}_{\varphi}^{\#} \mapsto A_{a} \in \mathscr{Y}(\mathcal{M})$.

In the following lemma, $\Phi$ denotes the representation of $\mathscr{S}(\mathcal{M})$ on $\mathscr{H} \otimes_{\mu \mathscr{L}} L^{2}(\mathscr{G}, v)$ defined in [Y1].

Lemma 2.10. The set $\left\{\Phi\left(A_{a}\right): a \in \mathscr{F}_{\varphi} \cap \mathscr{F}_{\varphi}^{\#}\right\}$ is a nondegenerate *-algebra.

Proof. It is obvious by the above remark that the set is a $*$-algebra. For showing nondegeneracy, we keep the notation in the proof of Lemma 2.5. We consider elements in $\mathscr{F}_{\varphi} \cap \mathscr{F}_{\varphi}^{\#}$ of the form $a_{1, f}\left(f \in \mathfrak{B}_{I}\right)$. We have

$$
\left(\Phi\left(A_{a_{1, f}}\right) \xi \mid \eta\right)=\iint f\left(\gamma_{1}\right)\left(u\left(\gamma_{1}\right) \xi\left(\gamma_{1}^{-1} \gamma\right) \mid \eta(\gamma)\right) d \lambda^{r(\gamma)}\left(\gamma_{1}\right) d v(\gamma)
$$

Note that $a_{1, f} * a_{1, g}=a_{1, f+g}\left(f, g \in \mathfrak{B}_{I}\right)$. This implies that we obtain a represen- 
tation of $\mathfrak{B}_{I}$ on $\mathscr{H} \otimes_{\mu \mathscr{L}} L^{2}(\mathscr{G}, v)$ through $\Phi: f \in \mathfrak{B}_{I} \mapsto \Phi\left(A_{a_{1 . f}}\right)$. We will now exhibit a useful interpretation of this representation. Let us consider a representation $\{u(\gamma) \otimes \lambda(\gamma)\}_{\gamma \in \mathscr{G}}$ of $\mathscr{G}$ on a Hilbert bundle $\left\{\mathscr{H}(x) \otimes L^{2}\left(\mathscr{G}^{x}, \lambda^{x}\right)\right\}_{x \in X}$ over $(X, \mu)$. Then, if one looks at (*) above carefully, it is not difficult to see that the representation $f \in \mathfrak{B}_{I} \mapsto \Phi\left(A_{a_{1, f}}\right)$ of $\mathfrak{B}_{I}$ is realized as the integrated representation $(u \otimes \lambda)(f)$ of the representation $\{u(\gamma) \otimes \lambda(\gamma)\}_{\gamma \in \mathscr{G}}$ of $\mathscr{G}$. Thus $\Phi\left(A_{a_{1 . t}}\right)=$ $(u \otimes \lambda)(f)$ is a nondegenerate representation of $\mathfrak{B}_{I}$. Therefore $\left\{\Phi\left(A_{a}\right)\right.$ : $\left.a \in \mathscr{F}_{\varphi} \cap \mathscr{F}_{\varphi}^{\#}\right\}$ is nondegenerate.

Q.E.D.

Theorem 2.11. $\widetilde{\mathfrak{T}}_{\varphi}$ becomes a left Hilbert algebra with respect to the following product and \#-operation;

$$
\begin{aligned}
\Lambda_{\varphi}(a) * \Lambda_{\varphi}(b) & =\Lambda_{\varphi}(a * b) \quad\left(a, b \in \mathscr{F}_{\varphi} \cap \mathscr{F}_{\varphi}^{\#}\right) \\
\Lambda_{\varphi}(a)^{\#} & =\Lambda_{\varphi}\left(a^{\#}\right) .
\end{aligned}
$$

$\widetilde{\mathfrak{P}}_{\varphi}$ has $\widetilde{J}_{\varphi}$ and $\widetilde{\Delta}_{\varphi}$ as its modular conjugation and modular operator.

Proof. In view of Proposition 2.3, left multiplication is continuous. It is an easy exercise to check that we have

$$
(\xi * \eta \mid \zeta)=\left(\eta \mid \xi^{\#} * \zeta\right)
$$

for any $\xi, \eta$ and $\zeta \in \widetilde{\Re}_{\varphi}$. By Lemma 2.9, the \#-operation $\xi \mapsto \xi^{\#}$ is preclosed. From Lemma 2.10 , it follows that $\widetilde{\mathfrak{T}}_{\varphi}^{2}$ is dense in $\widetilde{\mathfrak{T}}_{\varphi}$. Therefore $\widetilde{\mathfrak{T}}_{\varphi}$ is a left Hilbert algebra. The last assertion is due to Lemma 2.9 again.

Q.E.D.

Theorem 2.12. The left von Neumann algebra $\mathscr{R}_{\ell}\left(\widetilde{\mathfrak{N}}_{\varphi}\right)$ associated with $\widetilde{\mathfrak{N}}_{\varphi}$ is exactly the crossed product algebra $M \times{ }_{a} G$.

Proof. Obviously, $\mathscr{R}_{t}\left(\widetilde{\Re}_{\varphi}\right)$ is contained in the crossed product.

Suppose that $A \in \mathscr{Y}(\mathcal{M})$. Then there exists an element $a$ of $\mathscr{F}(\mathcal{M})$ such that $A(\gamma)=a(\gamma) u(\gamma) \otimes \lambda(\gamma)$ with $\left\|f_{a}\right\|_{I}<\infty$, where $f_{a}$ is, as usual, given by $f_{a}(\gamma)=$ $\|a(\gamma)\|$. Let $b \in \mathscr{F}_{\varphi} \cap \mathscr{F}_{\varphi}^{\#}$. Since $\left\|f_{b}\right\|_{I}<\infty$, we have $\left\|f_{a} * f_{b}\right\|_{I} \leq\left\|f_{a}\right\|_{I}\left\|f_{b}\right\|_{I}<\infty$. Moreover, the computation in the proof of Lemma 2.1 shows that, since $f_{a+b} \leq$ $f_{a} * f_{b}$, both $\lambda\left(f_{a, b}^{2}\right)$ and $\lambda^{\prime}\left(f_{a, b}^{2}\right)$ belong to $L^{x}(X, \mu)$. It follows that $a * b \in \mathscr{F}_{\varphi}$. Remark that $\Phi(A) \Phi\left(A_{b}\right)=\Phi\left(A_{a, b}\right)$. Since $\left\{\Phi\left(A_{a}\right): a \in \mathscr{F}_{\varphi} \cap \mathscr{F}_{\varphi}^{\#}\right\}$ is nondegenerate, we need only to prove, for the reverse inclusion, that every element of the form $\Phi\left(A_{a}\right)\left(a \in \mathscr{F}_{\varphi}\right)$ lies in $\mathscr{R}_{t}\left(\widetilde{\mathfrak{R}}_{\varphi}\right)$.

Suppose that $a \in \mathscr{F}_{\varphi}$. For any $n \geq 1$, define

$$
E_{n}=\left\{\gamma \in \mathscr{G}: \delta(\gamma) \in\left[n^{-1}, n\right] \text {, and }\|a(\gamma)\|,\left\|a\left(\gamma^{-1}\right)\right\| \leq n\right\} .
$$

Note that $E_{n}$ is symmetric. Namely, $\gamma \in E_{n}$ if and only if $\gamma^{-1} \in E_{n}$. Then we put

$$
a_{n}(\gamma)=1_{E_{n}}(\gamma) a(\gamma) \quad(\gamma \in \mathscr{G})
$$


We assert that each $a_{n}$ belongs to $\mathscr{F}_{\varphi} \cap \mathscr{F}_{\varphi}^{\#}$. For this we first observe that

$$
f_{a_{n}}(\gamma)=\left\|a_{n}\right\|=1_{E_{n}}(\gamma)\|a(\gamma)\| \leq\|a(\gamma)\|=f_{a}(\gamma),
$$

and that $a_{n}(\gamma)^{*} a_{n}(\gamma)=1_{E_{n}}(\gamma) a(\gamma)^{*} a(\gamma) \leq a(\gamma)^{*} a(\gamma)$. It easily follows that $a_{n} \in \mathscr{F}_{\varphi}$. Next we show that $a_{n}^{\#} \in \mathscr{F}_{\varphi}$. Since $E_{n}$ is symmetric, we have $f_{a_{n}^{*}}(\gamma)=$ $\left\|a_{n}^{\#}(\gamma)\right\|=1_{E_{n}}(\gamma) \delta(\gamma)^{-1}\left\|a\left(\gamma^{-1}\right)\right\|=1_{E_{n}}(\gamma) f_{a}^{\#}(\gamma) \leq f_{a}^{\#}(\gamma)$; so $\left\|f_{a_{n}^{\#}}\right\|_{I} \leq\left\|f_{a}^{\#}\right\|_{I}=$ $\left\|f_{a}\right\|_{I}<\infty$. Moreover we compute

$$
\begin{aligned}
\int f_{\mathrm{a}_{n}^{*}}^{2}(\gamma) d \lambda^{x}(\gamma) & =\int 1_{E_{n}}(\gamma) f_{a}^{\#}(\gamma)^{2} d \lambda^{x}(\gamma) \\
& =\int 1_{E_{n}}(\gamma) \delta(\gamma)^{-1} \delta(\gamma) f_{a}^{\#}(\gamma)^{2} d \lambda^{x}(\gamma) \\
& \leq n \int_{E_{n}} \delta(\gamma) f_{a}^{\#}(\gamma)^{2} d \lambda^{x}(\gamma) \\
& \leq n \int f_{a}(\gamma)^{2} d \lambda_{x}^{\prime}(\gamma) \\
& \leq n\left\|\lambda^{\prime}\left(f_{a}^{2}\right)\right\|_{\infty},
\end{aligned}
$$

and

$$
\begin{aligned}
\int f_{a_{n}^{*}}(\gamma)^{2} d \lambda_{x}^{\prime}(\gamma) & =\int 1_{E_{n}}(\gamma) f_{a}^{\#}(\gamma)^{2} \delta(\gamma) d \lambda_{x}(\gamma) \\
& =\int 1_{E_{n}}(\gamma) \delta(\gamma)^{-1} f_{a}\left(\gamma^{-1}\right)^{2} d \lambda_{x}(\gamma) \\
& \leq n \int E_{n} f_{a}\left(\gamma^{-1}\right)^{2} d \lambda_{x}(\gamma) \\
& \leq n \int f_{a}(\gamma)^{2} d \lambda^{x}(\gamma) \\
& \leq n\left\|\lambda\left(f_{a}^{2}\right)\right\|_{\infty} .
\end{aligned}
$$

These computations imply that $\lambda\left(f_{a_{n}^{*}}^{2}\right)$ and $\lambda^{\prime}\left(f_{a_{n}^{*}}^{2}\right)$ belong to $L^{\infty}(X, \mu)$. We also estimate the following;

$$
\begin{aligned}
\int \varphi_{s(\gamma)} \circ \alpha_{\gamma}^{-1}\left(a_{n}^{\#}(\gamma)^{*} a_{n}^{\#}(\gamma)\right) d v(\gamma)= & \int \delta(\gamma)^{-2} 1_{E_{n}}(\gamma) \varphi_{s(\gamma)}\left(a\left(\gamma^{-1}\right) a\left(\gamma^{-1}\right)\right) d v(\gamma) \\
\leq & \int\left(\int \delta(\gamma)^{-1} 1_{E_{n}}(\gamma)\left\|\varphi_{s(\gamma)}\right\|\right. \\
& \left.\left\|a\left(\gamma^{-1}\right)\right\|^{2} d \lambda_{x}(\gamma)\right) d \mu(x) \\
\leq & n \int\left(\int 1_{E_{n}}(\gamma)\left\|a\left(\gamma^{-1}\right)\right\|^{2} d \lambda_{x}(\gamma)\right)\left\|\varphi_{x}\right\| d \mu(x) \\
\leq & n \int\left(\int f_{a}(\gamma)^{2} d \lambda^{x}(\gamma)\right)\left\|\varphi_{x}\right\| d \mu(x)
\end{aligned}
$$




$$
\leq n\left\|\lambda\left(f_{a}^{2}\right)\right\|_{\infty}\|\varphi\|<\infty .
$$

Thus we conclude that $a_{n} \in \mathscr{F}_{\varphi} \cap \mathscr{F}_{\varphi}^{\#}$.

Finally we prove that $\Phi\left(A_{a}\right)$ belongs to $\mathscr{R}_{\ell}\left(\widetilde{\mathfrak{A}}_{\varphi}\right)$. Suppose that $\xi, \eta \in$ $\mathscr{H} \otimes_{\mu \circ} L^{2}(\mathscr{G}, v)$. Due to Lebesgue dominated convergence theorem, we have

$$
\begin{aligned}
\lim _{n \rightarrow \infty}\left(\Phi\left(A_{a_{n}}\right) \xi \mid \eta\right) & =\lim _{n \rightarrow \infty} \iint 1_{E_{n}}\left(\gamma_{1}\right)\left(a\left(\gamma_{1}\right) u\left(\gamma_{1}\right) \xi\left(\gamma_{1}^{-1} \gamma\right) \mid \eta(\gamma)\right) d \lambda^{r(\gamma)}\left(\gamma_{1}\right) d v(\gamma) \\
& =\iint\left(a\left(\gamma_{1}\right) u\left(\gamma_{1}\right) \xi\left(\gamma_{1}^{-1} \gamma\right) \mid \eta(\gamma)\right) d \lambda^{r(\gamma)}\left(\gamma_{1}\right) d v(\gamma) \\
& =\left(\Phi\left(A_{a}\right) \xi \mid \eta\right) .
\end{aligned}
$$

Hence $\Phi\left(A_{a}\right)$ lies in $\mathscr{R}_{\ell}\left(\widetilde{\mathfrak{I}}_{\varphi}\right)$ that is the weak closure of the *-algebra $\left\{\Phi\left(A_{a}\right)\right.$ : $\left.a \in \mathscr{F}_{\varphi} \cap \mathscr{F}_{\varphi}^{\#}\right\}$.

Q.E.D.

Definition 2.13. The faithful normal semifinite weight $\widetilde{\varphi}$ on $\mu \times{ }_{\alpha} \varphi$ associated with the left Hilbert algebra $\widetilde{\mathfrak{T}}_{\varphi}$ is called the dual weight of the originally given state $\varphi$ on $\mu$.

Theorem 2.14. The crossed product algebra $\mu \times{ }_{\alpha} G$ is generated by elements of the forms $a \otimes_{\mathscr{P}} 1(a \in \mathcal{M})$ and $(u \otimes \lambda)(f)\left(f \in \mathfrak{B}_{I}\right)$.

Proof. Let us denote by 2 the von Neumann algebra generated by operators of the above forms. Suppose that $b=\int_{X}^{\oplus} b(x) d \mu(x) \in \mathcal{M}$ and $f \in \mathfrak{B}_{I}$. Since

$$
\begin{aligned}
\left(\Phi\left(A_{a_{b, f}}\right) \xi \mid \eta\right) & =\iint f\left(\gamma_{1}\right)\left(b(r(\gamma)) u\left(\gamma_{1}\right) \xi\left(\gamma_{1}^{-1} \gamma\right) \mid \eta(\gamma)\right) d \lambda^{r(\gamma)}\left(\gamma_{1}\right) d v(\gamma) \\
& =\iint f\left(\gamma_{1}\right)\left(u\left(\gamma_{1}\right) \xi\left(\gamma_{1}^{-1} \gamma\right) \mid\left\{\left(b^{+} \otimes_{\mathscr{X}} 1\right) \eta\right\}(\gamma)\right) d \lambda^{r(\gamma)}\left(\gamma_{1}\right) d v(\gamma) \\
& =\left((u \otimes \lambda)(f) \xi \mid\left(b^{+} \otimes_{\mathscr{X}} 1\right) \eta\right) \\
& =\left(\left(b \otimes_{\mathscr{L}} 1\right)(u \otimes \lambda)(f) \xi \mid \eta\right),
\end{aligned}
$$

where $\xi, \eta \in \mathscr{H} \otimes_{\mu \circ g} L^{2}(\mathcal{G}, v)$, it follows that $\Phi\left(A_{a_{b, f}}\right)=\left(b \otimes_{x^{\prime}} 1\right)(u \otimes \lambda)(f)$. Upon taking $b=1$, we have $(u \otimes \lambda)(f) \in \mathcal{M} \times{ }_{\alpha} G$. Let $\left\{f_{i}\right\}$ be a net in $\mathfrak{B}_{I}$ such that the net $(u \otimes \lambda)\left(f_{i}\right)$ converges weakly to 1 . Then we have

$$
\begin{aligned}
& b \otimes_{\mathfrak{x}} 1=\text { weak-lim }\left(b \otimes_{\mathfrak{x}} 1\right)(u \otimes \lambda)\left(f_{i}\right) \\
& =\text { weak- } \lim _{i}^{i} \Phi\left(A_{a_{b, t_{t}}}\right) \text {. }
\end{aligned}
$$

This implies that $b \otimes_{\mathscr{T}} 1 \in \mathcal{M} \times{ }_{\alpha} \mathscr{G}$. Accordingly, 2 is contained in $\mu \times{ }_{\alpha} \mathscr{G}$. In order to prove that 2 indeed coincides with $\mu \times{ }_{\alpha} \varphi$, we show that a normal functional on $\mu \times{ }_{\alpha}{ } \mathcal{G}$ that vanishes on 2 must be zero. Let $\omega \in\left\{M \times{ }_{\alpha}{ }_{\alpha}\right\}_{+}$be such a functional. From Theorem 2.12, it follows that $\left\{\mu \times{ }_{\alpha} \mathscr{G}, \mathscr{H}_{\mathcal{H}} \otimes_{\mu x} L^{2}(\mathscr{G}, v)\right\}$ is a standard representation; so every normal functional on $\mu \times{ }_{\alpha}{ }^{\mathcal{G}}$ can be written as a vector functional. Thus there exist vectors $\xi, \eta$ in $\mathscr{H} \otimes_{\mu} L^{2}(\mathscr{G}, v)$ 
such that $\omega=\omega_{\xi, \eta}$. We write $\xi=\int_{X}^{\oplus} \xi_{x} d \mu(x), \eta=\int_{X}^{\oplus} \eta_{x} d \mu(x)$ according to the direct integral $\mathscr{H} \otimes_{\mu} L^{2}(\mathscr{G}, v)=\int_{X}^{\oplus} \mathscr{H}(x) \otimes L^{2}\left(G^{x}, \lambda^{x}\right) d \mu(x)$. Let $\left\{c_{n}\right\}_{n \geq 1}$ be a countable family of measurable fields of operators that appeared in the proof of Lemma 2.5. We denote by $\mathscr{P}_{0}$ the polynomial algebra over the rational complex field generated by $\left\{1, c_{n}, c_{m}^{+}\right\}_{n, m \geq 1}$, which is a countable subset of $\mathcal{M}$. Let $b \in \mathscr{P}_{0}$ and $f \in \mathfrak{B}_{l}$. Then, by Fubini's theorem, we have

$$
\begin{aligned}
0 & =<\Phi\left(A_{a_{b},}\right), \omega> \\
& =\left(\Phi\left(A_{a_{b},}\right) \xi \mid \eta\right) \\
& =\iint f\left(\gamma_{1}\right)\left(b\left(r\left(\gamma_{1}\right)\right) u\left(\gamma_{1}\right) \xi\left(\gamma_{1}^{-1} \gamma\right) \mid \eta(\gamma)\right) d \lambda^{r(\gamma)}\left(\gamma_{1}\right) d v(\gamma) \\
& =\int f\left(\gamma_{1}\right)\left(\int\left(b\left(r\left(\gamma_{1}\right)\right) u\left(\gamma_{1}\right) \xi\left(\gamma_{1}^{-1} \gamma\right) \mid \eta(\gamma)\right) d \lambda^{r\left(\gamma_{1}\right)}(\gamma)\right) d v\left(\gamma_{1}\right) .
\end{aligned}
$$

Since $f$ is an arbitrary element in $\mathfrak{B}_{I}$, it follows that there exists a $v$-null subset $N$ of $\mathscr{G}$ such that

$$
\int\left(b\left(r\left(\gamma_{1}\right)\right) u\left(\gamma_{1}\right) \xi\left(\gamma_{1}^{-1} \gamma\right) \mid \eta(\gamma)\right) d \lambda^{r\left(\gamma_{1}\right)}(\gamma)=0
$$

for all $\gamma \in \mathscr{G} \backslash N$. We may assume here that (i) this holds for any $b \in \mathscr{P}_{0}$, due to countability of $\mathscr{P}_{0}$; (ii) the set $\left\{b\left(r\left(\gamma_{1}\right)\right): b \in \mathscr{P}_{0}\right\}$ is weakly dense in $M\left(r\left(\gamma_{1}\right)\right)$ for any $\gamma_{1} \in \mathscr{G} \backslash N$. Note that identity (*) can be rewritten as

$$
\begin{aligned}
0 & =\int\left(b\left(r\left(\gamma_{1}\right)\right) u\left(\gamma_{1}\right) \xi_{s\left(\gamma_{1}\right)}\left(\gamma_{1}^{-1} \gamma\right) \mid \eta_{r\left(\gamma_{1}\right)}(\gamma)\right) d \lambda^{r\left(\gamma_{1}\right)}(\gamma) \\
& =\left(\left(b\left(r\left(\gamma_{1}\right)\right) \otimes 1\right)\left(u\left(\gamma_{1}\right) \otimes \lambda\left(\gamma_{1}\right)\right) \xi_{s\left(\gamma_{1}\right)} \mid \eta_{r\left(\gamma_{1}\right)}\right) \mathscr{\psi}_{\left(r\left(\gamma_{1}\right)\right) \otimes L^{2}\left(\xi^{\prime}\left(\nu_{1}\right), \lambda^{\prime}\left(\nu_{1}\right)\right)} .
\end{aligned}
$$

Let us fix one $\gamma_{1} \in \mathscr{G} \backslash N$. Since $\left\{b\left(r\left(\gamma_{1}\right)\right): b \in \mathscr{P}_{0}\right\}$ is weakly dense in $M\left(r\left(\gamma_{1}\right)\right)$, we have that $\left((b \otimes 1)\left(u\left(\gamma_{1}\right) \otimes \lambda\left(\gamma_{1}\right)\right) \xi_{s\left(\gamma_{1}\right)} \mid \eta_{r\left(\gamma_{1}\right)}\right)=0$ for any $b \in \mathcal{M}\left(r\left(\gamma_{1}\right)\right)$. Namely, we get

$$
\int\left(b u\left(\gamma_{1}\right) \xi\left(\gamma_{1}^{-1} \gamma\right) \mid \eta(\gamma)\right) d \lambda^{r\left(\gamma_{1}\right)}(\gamma)=0
$$

Now let $a \in \mathscr{F}_{\varphi} \cap \mathscr{F}_{\varphi}^{\#}$. Then, by Fubini's theorem, we have

$$
\begin{aligned}
<\Phi\left(A_{a}\right), \omega> & =\left(\Phi\left(A_{a}\right) \xi \mid \eta\right) \\
& =\iint\left(a\left(\gamma_{1}\right) u\left(\gamma_{1}\right) \xi\left(\gamma_{1}^{-1} \gamma\right) \mid \eta(\gamma)\right) d \lambda^{r(\gamma)}\left(\gamma_{1}\right) d v(\gamma) \\
& =\iint\left(a\left(\gamma_{1}\right) u\left(\gamma_{1}\right) \xi\left(\gamma_{1}^{-1} \gamma\right) \mid \eta(\gamma)\right) d \lambda^{r\left(\gamma_{1}\right)}(\gamma) d v\left(\gamma_{1}\right)=0 .
\end{aligned}
$$

The last equality is due to $(* *)$. Since $\left\{\Phi\left(A_{a}\right): a \in \mathscr{F}_{\varphi} \cap \mathscr{F}_{\varphi}^{\#}\right\}$ is $\sigma$-weakly dense in $M \times{ }_{\alpha} \xi$, we have that $\omega=0$. Therefore the algebra 2 coincides with the crossed product algebra.

Q.E.D. 
Corollary 2.15. The commutant $\left\{\mu \times{ }_{\alpha} \mathcal{G}\right\}^{\prime}$ of the crossed product is generated by elements of the forms $\alpha^{\prime}\left(b^{\prime}\right)\left(b^{\prime} \in \mathcal{M}^{\prime}\right)$ and $1 \otimes_{\mathscr{L}} \rho(f)\left(f \in \mathfrak{B}_{I}\right)$, where

$$
\left\{\alpha^{\prime}\left(b^{\prime}\right) \xi\right\}(\gamma)=u(\gamma) b^{\prime}(s(\gamma)) u(\gamma)^{+} \xi(\gamma) \quad\left(\xi \in \mathscr{H} \otimes_{\mu \mathscr{L}} L^{2}(\xi, v)\right)
$$

and $\rho$ is the right regular representation of $\mathscr{G}$, that is,

$$
\rho(f) g=g * f \quad\left(f, g \in \mathfrak{B}_{I}\right) .
$$

Proof. By the preceding theorem, the commutant is generated by elements of the forms $\widetilde{J}_{\varphi}\left(a \otimes_{\mathscr{L}} 1\right) \widetilde{J}_{\varphi}(a \in \mathcal{M})$ and $\widetilde{J}_{\varphi}(u \otimes \lambda)(f) \widetilde{J}_{\varphi} \quad\left(f \in \mathcal{B}_{l}\right)$. Let $\xi \in$ $\mathscr{H} \otimes_{\mu} L^{2}(G, v)$. Then

$$
\begin{aligned}
& \left\{\widetilde{J}_{\varphi}\left(a \otimes_{\mathscr{L}} 1\right) \widetilde{J}_{\varphi} \xi\right\}(\gamma)=\delta(\gamma)^{-1 / 2} u(\gamma) J_{\varphi_{s(\gamma)}}\left\{\left(a \otimes_{\mathscr{L}} 1\right) \widetilde{J}_{\varphi} \xi\right\}\left(\gamma^{-1}\right) \\
& =\delta(\gamma)^{-1 / 2} u(\gamma) J_{\varphi_{s(\omega)}} a(s(\gamma))\left\{\widetilde{J}_{\varphi} \xi\right\}\left(\gamma^{-1}\right) \\
& =\delta(\gamma)^{-1 / 2} u(\gamma) J_{\varphi_{s(\%)}} a(s(\gamma)) \delta(\gamma)^{1 / 2} J_{\varphi_{s(*)}} u(\gamma)^{4} \xi(\gamma) \\
& =u(\gamma) J_{\varphi_{s(\nu)}} a(s(\gamma)) J_{\varphi_{s(\nu)}} u(\gamma)^{+} \xi(\gamma) \text {. }
\end{aligned}
$$

Put $b^{\prime}=J_{\varphi} a J_{\varphi}=\int_{X}^{\oplus} J_{\varphi_{1}} a(x) J_{\varphi_{\curlywedge}} d \mu(x) \in \mathcal{M}^{\prime}$. Then the above computation shows that

$$
\widetilde{J}_{\varphi}\left(a \otimes_{\mathscr{L}} 1\right) \widetilde{J}_{\varphi}=\alpha^{\prime}\left(b^{\prime}\right)=\alpha^{\prime}\left(J_{\varphi} a J_{\varphi}\right)
$$

Moreover

$$
\begin{aligned}
\left\{\widetilde{J}_{\varphi}(u \otimes \lambda)(f) \widetilde{J}_{\varphi} \xi\right\}(\gamma)= & \delta(\gamma)^{-1 / 2} J_{\varphi_{1(\nu)}} u(\gamma)\left\{(u \otimes \lambda)(f) \widetilde{J}_{\varphi} \xi\right\}\left(\gamma^{-1}\right) \\
= & \delta(\gamma)^{-1 / 2} J_{\varphi((\gamma)} u(\gamma) \int f\left(\gamma_{1}\right) u\left(\gamma_{1}\right) \\
& \left\{\widetilde{J}_{\varphi} \xi\right\}\left(\gamma_{1}^{-1} \gamma^{-1}\right) d \lambda^{s(\gamma)}\left(\gamma_{1}\right) \\
= & \int \delta\left(\gamma_{1}\right)^{1 / 2} f\left(\gamma_{1}\right) \xi\left(\gamma_{1} \gamma\right) d \lambda^{s(\gamma)}\left(\gamma_{1}\right) \\
= & \int\left\{\Delta^{-1 / 2} \bar{f}^{b}\right\}\left(\gamma_{1}^{-1}\right) \xi\left(\gamma_{1} \gamma\right) d \lambda^{s(\gamma)}\left(\gamma_{1}\right) \\
= & \left\{\left(1 \otimes_{\mathscr{L}} \rho\left(\Delta^{-1 / 2} \bar{f}^{b}\right)\right) \xi\right\}(\gamma),
\end{aligned}
$$

which implies that

$$
\widetilde{J}_{\varphi}(u \otimes \lambda)(f) \widetilde{J}_{\varphi}=1 \otimes_{x} \rho\left(\Delta^{-1 / 2} \bar{f}^{b}\right) .
$$

Thus our assertion follows.

Q.E.D.

We close this section with the following lemma which tells us how the modular automorphism group of the dual weight $\widetilde{\varphi}$ acts on the crossed product. Since our proof involves only a simple calculation, we leave verification of the assertion to readers. 
Lemma 2.16. Let $a \in M$ and $f \in B_{I}$. Then we have

$$
\sigma_{t}^{\widetilde{\varphi}}\left(a \otimes_{\varkappa} 1\right)=\sigma_{t}^{\varphi}(a) \otimes_{o_{L}} 1
$$

and

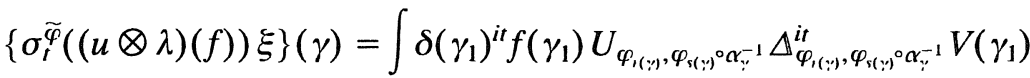

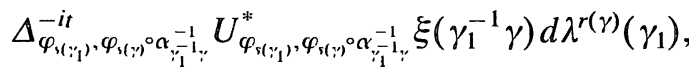

where $\xi \in \mathscr{H} \otimes_{\mu \mathscr{L}} L^{2}(\mathscr{G}, v)$.

\section{§3. Relative Commutants in a Crossed Product}

In this section, we compute relative commutants of various kinds of subalgebras of a crossed product (by a groupoid action).

Before we state our results, we need to introduce a notion of a fixed point algebra of a groupoid action.

Definition 3.1. Let $\left(\mathscr{G},\{M(x)\}_{x \in X},\left\{\alpha_{\gamma}\right\}_{\gamma \in \mathscr{G}}\right)$ be a groupoid action. Then the fixed point algebra of the given action is a set $\mathcal{M}^{\alpha}$ of all elements $a=\int_{X}^{\oplus}$ $a(x) d \mu(x)$ in $\mu=\int_{X}^{\oplus} M(x) d \mu(x)$ such that $\alpha_{\gamma}(a(s(\gamma)))=a(r(\gamma))$ for $v$-a.e. $\gamma \in \mathscr{G}$. It is clear that $\mathcal{M}^{\alpha}$ is a von Neumann subalgebra of $\mathcal{M}$.

We still keep our previous notations in the present section.

Theorem 3.2. Let $\left(\mathscr{G},\{M(x)\}_{x \in X},\left\{\alpha_{\gamma}\right\}_{\gamma \in \mathscr{G}}\right)$ be an action of $\mathscr{G}$ as before. We assume that $\mathscr{G}$ is a principal groupoid. Then

(i) $\left\{c \otimes_{\mathscr{L}} 1: c \in \mathscr{L}(\mathcal{M})\right\}^{\prime} \cap \mathcal{M} \times_{\alpha} \mathscr{G}=\left\{a \otimes_{\mathscr{L}} 1: a \in M\right\}$,

where $\mathscr{L}(\mathcal{N})$ hereafter denotes the center of a von Neumann algebra $\mathcal{N}$.

(ii) $\left\{a \otimes_{\mathscr{L}} 1: a \in M\right\}^{\prime} \cap M \times_{\alpha} \mathscr{G}=\left\{c \otimes_{\mathscr{W}} 1: c \in \mathscr{L}(\mathcal{M})\right\}$.

(iii) $\mathscr{L}\left(\mathcal{M} \times{ }_{\alpha} \mathscr{G}\right)=\left\{c \otimes_{\mathscr{L}} 1: c \in \mathscr{L}(\mathcal{M})^{\alpha}\right\}$,

where $\mathscr{L}(M)^{\alpha}$, of course, denotes the fixed point algebra of an action ( $G$, $\left.\{\mathscr{L}(\mathcal{M}(x))\},\left\{\alpha_{\gamma}\right\}\right)$.

(iv) The center $\mathscr{L}\left(\left(M \times_{\alpha} \mathscr{G}\right)_{\widetilde{\varphi}}\right)$ of the centralizer $\left(M \times_{\alpha} \mathscr{G}\right)_{\widetilde{\varphi}}$ of the dual weight $\widetilde{\varphi}$ is contained in the algebra $\left\{a \otimes_{\mathscr{L}} 1: a \in \mathscr{L}\left(\mathcal{M}_{\varphi}\right)\right\}$

Proof. (i) Clearly, the right hand side is contained in the other. For the reverse inclusion, let $Y$ be a member of the crossed product commuting with $\mathscr{L}(M) \otimes_{\mathscr{L}}$ C. From Corollary 2.15, it follows that $Y$ satisfies (2.10) and (2.11) of [Y2]. Hence $Y$ belongs to the commutant of $\mathbf{C} \otimes_{\mathscr{E}} \mathscr{R}(\mathscr{G})^{\prime}$. Since operators of the type $1 \otimes_{\mathscr{X}} M(h \circ s)\left(h \in L^{\propto}(X, \mu)\right)$ lie in $\mathbf{C} \otimes_{\mathscr{L}} \mathscr{R}(\mathscr{G})^{\prime}$, we have 


$$
\left[Y, 1 \otimes_{\mathscr{L}} M(h \circ s)\right]=0 \quad\left(h \in L^{\infty}(X, \mu)\right) .
$$

Let us note that, if $k \in L^{\infty}(X, \mu)$, then $1 \otimes_{\mathscr{W}} M(k \circ r) \subseteq\left\{c \otimes_{\mathscr{L}} 1: c \in \mathscr{L}(\mathcal{M})\right\}$; so we also have

$$
\left[Y, 1 \otimes_{\mathscr{Q}} M(k \circ r)\right]=0 \quad\left(k \in L^{\infty}(X, \mu)\right) .
$$

Since $\mathscr{G}$ is principal, the algebras $\mathscr{L}_{R}$ and $\mathscr{L}_{S}$ generate $L^{\infty}(\mathscr{G}, v)$. Thus, in view of (3.3) and (3.4), $Y$ satisfies (2.9) of [Y2]. Then we apply the argument in the proof of Theorem 2.6 of [Y2] to our operator $Y$ so as to conclude that $Y$ must be of the form $Y=a \otimes_{\mathscr{q}} 1(a \in \mathcal{M})$.

(ii) It suffices to show that the left hand side is contained in the other. By (i), we have

$$
\begin{aligned}
\left\{a \otimes_{\mathscr{L}} 1: a \in \mathcal{M}\right\}^{\prime} \cap \mathcal{M} \times{ }_{a} G \subseteq\left\{c \otimes_{\mathscr{W}} 1: c \in \mathscr{L}(\mathcal{M})\right\}^{\prime} \cap \mathcal{M} \times{ }_{\alpha} G \\
\\
=\left\{a \otimes_{\mathscr{W}} 1: a \in \mathcal{M}\right\} .
\end{aligned}
$$

This proves (ii).

(iii) By (ii), we have

$$
\begin{aligned}
\mathscr{L}\left(\mathcal{M} \times_{\alpha} \mathscr{G}\right) & \subseteq\left\{a \otimes_{\mathscr{L}} 1: a \in \mathcal{M}\right\}^{\prime} \cap M \times_{\alpha} \mathscr{G} \\
& =\left\{c \otimes_{\mathscr{L}} 1: c \in \mathscr{W}(\mathcal{M})\right\} .
\end{aligned}
$$

Thus every element in $\mathscr{L}\left(\mathcal{M} \times{ }_{\alpha} \mathscr{G}\right)$ has the form $c \otimes_{\mathscr{L}} 1(c \in \mathscr{L}(\mathcal{M}))$. Note that the condition that $\left[c \otimes_{\mathscr{o}} 1,(u \otimes \lambda)(f)\right]=0$ for all $f \in \mathcal{B}_{I}$, is obviously necessary and sufficient for an element $c \otimes_{\mathscr{L}} 1(c \in \mathscr{L}(\mathcal{M}))$ to belong to $\mathscr{L}\left(\mathcal{M} \times{ }_{\alpha} \mathscr{G}\right)$. Recall that $(u \otimes \lambda)(f)$ is the integrated representation of a (groupoid) representation $\{u(\gamma) \otimes \lambda(\gamma)\}_{\gamma \in \mathcal{G}}$ of $\mathscr{G}$ on the Hilbert bundle $\left\{\mathscr{H}(x) \otimes L^{2}\left(\mathscr{G}^{x}, \lambda^{x}\right)\right\}_{x \in X}$. It is known that the commutant of $\left\{(u \otimes \lambda)(f): f \in \mathfrak{B}_{I}\right\}$ is the algebra of decomposable operators $R$ such that $R(r(\gamma))(u(\gamma) \otimes \lambda(\gamma))=(u(\gamma) \otimes \lambda(\gamma)) R(s(\gamma))$ for $v$-a.e. $\gamma \in \mathscr{G}$. It now easily follows that, for $c \in \mathscr{L}(\mathcal{M})$, we have

$$
\begin{aligned}
& {\left[c \otimes_{\mathscr{L}} 1,(u \otimes \lambda)(f)\right]=0 \quad\left(f \in \mathfrak{B}_{l}\right)} \\
& \Leftrightarrow(c(r(\gamma)) \otimes 1)(u(\gamma) \otimes \lambda(\gamma))=(u(\gamma) \otimes \lambda(\gamma))(c(s(\gamma)) \otimes 1) \text { for } v \text {-a.e. } \gamma \in \mathscr{G} \\
& \Leftrightarrow c(r(\gamma)) u(\gamma)=u(\gamma) c(s(\gamma)) \quad \text { for } v \text {-a.e. } \gamma \in \mathscr{G} \\
& \Leftrightarrow \alpha_{\gamma}(c(s(\gamma)))=c(r(\gamma)) \quad \text { for } v \text {-a.e. } \gamma \in \mathscr{G} \\
& \Leftrightarrow c \in \mathscr{L}(\mathcal{M})^{\alpha} .
\end{aligned}
$$

(iv) Let $R \in \mathscr{Z}\left(\left(M \times{ }_{a} \varphi\right)_{\widetilde{\varphi}}\right)$. Due to Lemma 2.16, we know that $\mu_{\varphi} \otimes_{\mathscr{Q}} \mathbf{C}$ is contained in $\left(M \times{ }_{\alpha} \mathscr{G}\right)_{\widetilde{\varphi}}$. In particular, $\mathscr{Z}(M) \otimes_{\mathscr{Z}} \mathbf{C}$ is a subalgebra of $\left(M \times{ }_{\alpha} \mathscr{G}\right)_{\widetilde{\varphi}}$. Thus $R$ commutes with $\mathscr{L}(\mathcal{M}) \otimes_{\mathscr{L}} \mathbf{C}$. Accordingly, $R$ is of the form $R=a \otimes_{\mathscr{E}} 1$ $(a \in \mathcal{M})$ by (i). Again, by Lemma 2.16, the element $a$ must lie in $\mathcal{M}_{\varphi}$. It is now easy to see that $a$ is in $\mathscr{E}\left(\mathcal{M}_{\varphi}\right)$.

Q.E.D. 


\section{§4. The T-set of a Crossed Product}

This section is devoted to computing the T-set of the crossed product algebra by a groupoid action.

As usual, we fix an action $\left(\mathscr{G},\{\mathcal{M}(x)\},\left\{\alpha_{\gamma}\right\}\right)$ of a measured groupoid $\mathscr{G}$ and a faithful normal state $\varphi$ on $\mathcal{M}$. The notations introduced so far are retained in this section too. It should be mentioned that computation of the T-set of a crossed product heavily depends on the results established in the preceding section. So we need to assume that our groupoid $\mathscr{G}$ is principal. For a von Neumann algebra $\mathcal{N}$, let $T(\mathcal{N})$ denote the T-set on $\mathcal{N}$ in the sense of Connes [C1].

We define a subset $T(\mathscr{G}, \mathcal{M}, \alpha)$ of $\mathbf{R}$ to be the set of real numbers $t$ such that (T1) $t \in T(M)$

(T2) $\left(D \varphi_{r(\gamma)}: D \varphi_{s(\gamma)} \circ \alpha_{\gamma}^{-1}\right)_{t}=\delta(\gamma)^{-i t} w(r(\gamma)) \alpha_{\gamma}\left(w(s(\gamma))^{+}\right)$for $v$-a.e. $\gamma \in \mathscr{G}$, where $w=\int_{X}^{\oplus} w(x) d \mu(x) \in \mathcal{M}$ is a unitary satisfying $\sigma_{t}^{\varphi}=\mathrm{Ad} w$.

Remark 4.1. In (T2), $w$ satisfies $\sigma_{t}^{\varphi}=\operatorname{Ad} w$; so we have $\sigma_{t}^{\varphi_{1}}=\operatorname{Ad} w(x)$ for $\mu$-a.e. $x \in X$. It then follows that $\sigma_{t}^{\varphi_{s(\nu)}}=\operatorname{Ad} w(s(\gamma))$ and $\sigma_{t}^{\varphi_{1}(())}=\operatorname{Ad} w(r(\gamma))$ for $v$-a.e. $\gamma \in \mathscr{G}$.

Theorem 4.2. If $\mathscr{G}$ is principal, then we have

$$
T(\mathscr{G}, \mathcal{M}, \alpha)=T\left(\mathcal{M} \times{ }_{a} \mathscr{G}\right) .
$$

Proof. Let $t \in T(\mathscr{G}, \mathcal{M}, \alpha)$ and take a unitary $w \in \mathcal{M}$ in (T2). By Lemma 2.16 , it is easy to check that $\sigma_{t}^{\widetilde{\varphi}}=\operatorname{Ad}\left(w \otimes{ }_{*} 1\right)$; so we have $t \in T\left(\mathcal{M} \times{ }_{a} \mathscr{G}\right)$.

Next suppose that we take $t \in T\left(M \times{ }_{\alpha} \mathscr{G}\right)$. Then there exists a unitary in $\mathcal{M} \times{ }_{\alpha} \mathscr{G}$ implementing $\sigma_{t}^{\widetilde{\varphi}}$, which we may assume belongs to $\mathscr{L}\left(\left(\mathcal{M} \times_{\alpha} \mathscr{G}\right)_{\widetilde{\varphi}}\right)$. By Lemma 3.2 (iv), the unitary has the form $w \otimes_{\mathscr{L}} 1$, where $w \in \mathscr{L}\left(\mathcal{M}_{\varphi}\right)$. From Lemma 2.16, it follows that $\sigma_{t}^{\varphi}=\operatorname{Ad} w$. Thus $t \in T(M)$. For $f \in \mathfrak{B}_{l}$, we compute

$$
\begin{aligned}
\left\{\sigma_{t}^{\widetilde{\varphi}}((u \otimes \lambda)(f)) \xi\right\}(\gamma) & =\left\{\left(w \otimes_{\mathscr{W}} 1\right)(u \otimes \lambda)(f)\left(w^{+} \otimes_{\mathscr{\varkappa}} 1\right) \xi\right\}(\gamma) \\
& =\int f\left(\gamma_{1}\right) w(r(\gamma)) \alpha_{\gamma_{1}}\left(w\left(s\left(\gamma_{1}\right)\right)^{+}\right) u\left(\gamma_{1}\right) \xi\left(\gamma_{1}^{-1} \gamma\right) d \lambda^{r(\gamma)}\left(\gamma_{1}\right) .
\end{aligned}
$$

If we compare this with the second assertion of Lemma 2.16, then we obtain

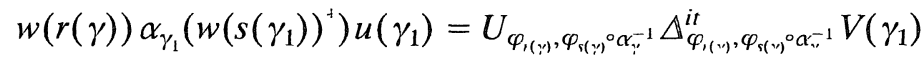

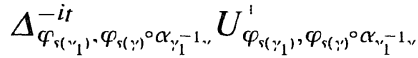

for $v$-a.e. $\gamma \in \mathscr{G}$ and $\lambda^{r(\gamma)}$-a.e. $\gamma_{1}$ in $\mathscr{G}^{r(\gamma)}$. From this, it follows that, for $v$-a.e. $\gamma \in \mathscr{G}$ and $\lambda^{r(\gamma)}$-a.e. $\gamma_{1}$ in $\mathscr{G}^{r(\gamma)}$,

$$
\begin{aligned}
& \delta\left(\gamma_{1}\right)^{-i t} w(r(\gamma)) \alpha_{\gamma_{1}}\left(w\left(s\left(\gamma_{1}\right)\right)^{+}\right)
\end{aligned}
$$

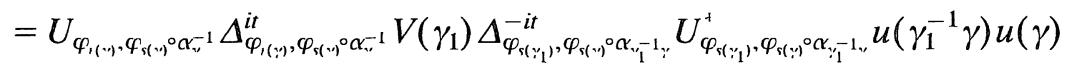




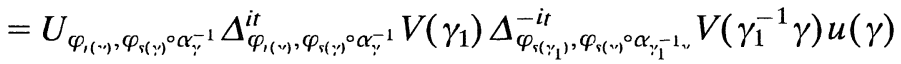

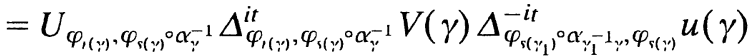

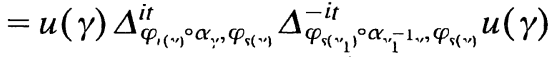

$$
\begin{aligned}
& =\left(D \varphi_{r(\gamma)}: D \varphi_{s\left(\gamma_{1}\right)}{ }^{\circ} \alpha_{\gamma_{1}}^{-1}\right)_{t} \text {. }
\end{aligned}
$$

Accordingly, we have

$$
\left(D \varphi_{r(\gamma)}: D \varphi_{s(\gamma)}{ }^{\circ} \alpha_{\gamma}^{-1}\right)_{t}=\delta(\gamma)^{-i t} w(r(\gamma)) \alpha_{\gamma}\left(w(s(\gamma))^{+}\right)
$$

for $v$-a.e. $\gamma \in \mathscr{G}$. Thus $t \in T(\mathscr{G}, \mathcal{M}, \alpha)$.

Q.E.D.

\section{§5. The Smooth Flow of Weights of a Crossed Product}

This section is concerned with computation of the (smooth) flow of weights of the crossed product by a groupoid action.

It is known (see [C\&T]) that the (smooth) flow of weights of a von Neumann algebra $\mathcal{N}$ can be realized as the restriction of the dual action to the center of the crossed product by a modular action on $\mathcal{N}$. It should be noted that this realization is independent of the choice of a faithful normal semifinite weight on the original algebra $\mathcal{N}$. Hence, in order to compute the flow of weights of a groupoid crossed product, we may consider the modular action on it derived from a dual weight.

Let $\left(\mathscr{G},\{\mathcal{M}(x)\},\left\{\alpha_{\gamma}\right\}\right)$ be an action and $\varphi$ be a faithful normal state on $\mathcal{M}$ as before. Also in this section, we keep our previous notations. As we have explained now, we look at the crossed product $\mathscr{P}=\left(\mathcal{M} \times{ }_{a} \mathscr{G}\right) \times{ }_{\sigma^{4}} \mathbb{R}$. However it is very difficult, only by looking at this algebra $\mathscr{P}$, to grasp the center $\mathscr{E}(\mathscr{P})$, which is the very algebra we are most interested in. In order to circumvent the difficulty, we will employ a standard method (in the case of group actions) in which we construct another action of the original groupoid $\mathscr{G}$ whose crossed product is isomorphic to $\mathscr{P}$. Then our next step is to resort to the results established in $\S 4$ so as to capture the center of the crossed product.

To make our theory consistent, we change definitions of the crossed product of a von Neumann algebra by an action of a locally compact group. Our definition is as follows. Let $\alpha: G \mapsto \operatorname{Aut}(\mathcal{N})$ be an action of a locally compact group $G$ on a von Neumann algebra $\mathcal{N}$. Suppose that $\{\mathcal{N}, \mathscr{K}\}$ is a standard representation. Let $u(g)$ be the unique unitary on $\mathcal{K}$ implementing $\alpha_{g}^{\prime}$, that is, $\alpha_{g}=\operatorname{Ad} u(g)$. Then our definition of a crossed product $\mathcal{N} \times{ }_{a} G$ is the von Neumann algebra generated by operators $y \otimes 1(y \in \mathcal{N})$ and $u(g) \otimes \lambda(g)(g \in G)$, where $\lambda(\cdot)$ denotes the left regular representation of $G$. Remark that our crossed product is spatially isomorphic to the conventional one defined in [T2]. If we adopt this definition, then we have that

$$
\mathscr{P}=\left(\mathcal{M} \times_{\alpha} \mathscr{G}\right) \otimes \mathbf{C} \vee\left\{\widetilde{\Delta}_{\varphi}^{i t} \otimes \lambda(t): t \in \mathbf{R}\right\}^{\prime \prime} .
$$


Combining this with Theorem 2.14 , we obtain

Lemma 5.1. The algebra $\mathscr{P}$ is generated by operators $\left(a \otimes_{\mathscr{L}} 1\right) \otimes 1(a \in \mathcal{M})$, $(u \otimes \lambda)(f) \otimes 1\left(f \in \mathfrak{B}_{I}\right)$ and $\widetilde{\Delta}_{\varphi}^{i t} \otimes \lambda(t)(t \in \mathbf{R})$.

Our next objective is, as we stated, to realize the algebra $\mathscr{P}$ as the crossed product by an action of $\mathscr{G}$. For this purpose, we consider a family of von Neumann algebras $\left\{\mathcal{N}(x)=\mathcal{M}(x) \times_{\sigma^{\prime \prime},} \mathbf{R}\right\}_{x \in X}$ on $\left\{\mathscr{H}(x) \otimes L^{2}(\mathbf{R})\right\}_{x \in X}$. It is easy to see that $\left\{\mathcal{N}(x), \mathscr{H}(x) \otimes L^{2}(\mathbf{R})\right\}_{x \in X}$ is a measurable field of von Neumann algebras over $(X, \mu)$.

For each $\gamma \in \mathscr{G}$, we define a unitary $W(\gamma)$ from $\mathscr{H}(s(\gamma)) \otimes L^{2}(\mathbb{R})$ onto $\mathscr{H}(r(\gamma)) \otimes L^{2}(\mathbf{R})$ by

$$
\{W(\gamma) \xi\}(t)=\delta(\gamma)^{-i t} \Delta_{\varphi_{,(\gamma)}}^{i t}\left(D \varphi_{r(\gamma)}: D \varphi_{s(\gamma)}{ }^{\circ} \alpha_{\gamma}^{-1}\right)_{-t} u(\gamma) \Delta_{\varphi_{s(\nu)}}^{-i t} \xi(t),
$$

where $\xi \in \mathscr{H}(s(\gamma)) \otimes L^{2}(\mathbf{R})=L^{2}(\mathbf{R}, \mathscr{H}(s(\gamma)))$. It is readily verified that $W(\gamma)$ is indeed a unitary and its adjoint is given by

$$
\left\{W(\gamma)^{+} \eta\right\}(t)=\delta(\gamma)^{i t} \Delta_{\varphi_{s(\vartheta)}}^{i t} u(\gamma)^{+}\left(D \varphi_{r(\gamma)}: D \varphi_{s(\gamma)}{ }^{\circ} \alpha_{\gamma}^{-1}\right)_{-t}^{+} \Delta_{\varphi_{l(:)}}^{-i t} \eta(t),
$$

where $\eta$ is, of course, in $\mathscr{H}(r(\gamma)) \otimes L^{2}(\mathbf{R})=L^{2}(\mathbf{R}, \mathscr{H}(r(\gamma)))$. We assert that Ad $W(\gamma)$ carries $\mathcal{N}(s(\gamma))$ onto $\mathcal{N}(r(\gamma))$. Indeed, we have

$$
\begin{gathered}
W(\gamma)(a \otimes 1) W(\gamma)^{+}=\alpha_{\gamma}(a) \otimes 1 \quad(a \in \mathcal{M}(s(\gamma))) \\
W(\gamma)\left(\Delta_{\varphi_{(,(;)}}^{i t} \otimes \lambda(t)\right) W(\gamma)^{+}=\delta(\gamma)^{-i t}\left(D \varphi_{s(\gamma)}{ }^{\circ} \alpha_{\gamma}^{-1}: D \varphi_{r(\gamma)}\right)_{t} \Delta_{\varphi_{(,(j)}}^{i t} \otimes \lambda(t) .
\end{gathered}
$$

Moreover it can be shown that $W(\gamma)$ is a (measurable) groupoid representation of $\mathscr{G}$ on the Hilbert bundle $\left\{\mathscr{H}(x) \otimes L^{2}(\mathbf{R})\right\}_{x \in X}$. Thus we obtain a new action $\left(\mathscr{G},\{\mathcal{N}(x)\}_{x \in X},\left\{\bar{\alpha}_{\gamma}=\operatorname{Ad} W(\gamma)\right\}_{\gamma \in \mathscr{G}}\right)$ of the original groupoid $\mathscr{G}$. We set

$$
\mathcal{N}=\int_{X}^{\oplus} \mathcal{N}(x) d \mu(x)=\mathcal{M} \times \sigma_{\sigma^{\prime \prime}} \mathbf{R} .
$$

We consider the crossed product $\mathcal{N} \times \frac{-}{\alpha}$ by the new action of $\mathscr{G}$. Let $(W \otimes \lambda)(\cdot)$ denote the integrated representation of the groupoid representation $\{W(\gamma) \otimes$ $\lambda(\gamma)\}$ on the Hilbert bundle $\left\{\mathscr{H}(x) \otimes L^{2}(\mathbf{R}) \otimes L^{2}\left(\mathscr{G}^{x}, \lambda^{x}\right)\right\}_{x \in X}$ over $(X, \mu)$. Then it follows from Theorem 2.14 that the crossed product $\mathcal{N} \times{ }_{\bar{\alpha}} \mathscr{G}$ is generated by operators $Y \otimes_{\mathscr{L}} 1(Y \in \mathcal{N})$ and $(W \otimes \lambda)(f)\left(f \in \mathfrak{B}_{I}\right)$. Since $\mathcal{N}$ itself is generated by $a \otimes 1(a \in \mathcal{M})$ and $\Delta_{\varphi}^{i t} \otimes \lambda(t)(t \in \mathbf{R})$, we obtain

Lemma 5.4. The crossed product $2=\mathcal{N} \times \frac{-G}{\alpha}$ is generated by operators $(a \otimes 1) \otimes_{w_{\mathcal{L}}} 1(a \in \mathcal{M}),\left(\Delta_{\varphi}^{i t} \otimes \lambda(t)\right) \otimes_{\mathscr{L}} 1(t \in \mathbf{R})$ and $(W \otimes \lambda)(f)\left(f \in \mathfrak{B}_{I}\right)$.

Proposition 5.5. The von Neumann algebra $\mathscr{P}=\left(\mathcal{M} \times \alpha_{\alpha} \mathscr{G}\right) \times{ }_{\sigma^{\widetilde{4}}} \mathbf{R}$ is spatially isomorphic to the crossed product $\mathscr{Q}=\left(\mathcal{M} \times_{\sigma^{4}} \mathbf{R}\right) \times{ }_{\bar{\alpha}} \varphi$. 
Proof. The algebra $\mathscr{P}$ is acting on $\left(\mathscr{H} \otimes_{\mu \mathscr{L}} L^{2}(\mathscr{G}, v)\right) \otimes L^{2}(\mathbf{R})$ and $\mathscr{Q}$ is acting on $\left(\mathscr{H} \otimes L^{2}(\mathbf{R})\right) \otimes_{\mu \mathscr{L}} L^{2}(\mathscr{G}, v)$. Note that these Hilbert spaces are canonically isomorphic to each other; so we regard them as the same Hilbert space $\mathscr{I}$, which can be viewed as the set of all measurable functions $\xi$ on $\mathscr{G} \times \mathbf{R}$ into $\Pi_{x \in X}$ $\mathscr{H}(x)$ such that (i) $\xi(\gamma, t) \in \mathscr{H}(r(\gamma))$ (ii) $\int\|\xi(\gamma, t)\|^{2} d(v \times m)(\gamma, t)<\infty$, where $m$ is the Lebesgue measure on $\mathbf{R}$. We define a unitary $K$ on $\Phi$ by

$$
\{K \xi\}(\gamma, t)=\delta(\gamma)^{i t} u(\gamma)\left(D \varphi_{r(\gamma)} \circ \alpha_{\gamma}: D \varphi_{s(\gamma)}\right)_{t} \Delta_{\varphi_{s(\gamma)}}^{i t} u(\gamma)^{+} \Delta_{\varphi_{1(*)}}^{-i t} \xi(\gamma, t) .
$$

The adjoint $K^{4}$ is given by

$$
\left\{K^{+} \xi\right\}(\gamma, t)=\delta(\gamma)^{-i t} \Delta_{\varphi_{(\gamma(\gamma)}}^{i t} u(\gamma) \Delta_{\varphi_{s(\gamma)}}^{-i t}\left(D \varphi_{r(\gamma)}{ }^{\circ} \alpha_{\gamma}: D \varphi_{((*))}\right)_{t}^{+} u(\gamma)^{+} \xi(\gamma, t) .
$$

Thus $K$ is in fact a unitary. It is only a matter of computation to verify the following identities

$$
\begin{aligned}
\left(a \otimes_{x^{*}} 1\right) \otimes 1 & =K\left((a \otimes 1) \otimes_{\left.{ }_{x} 1\right) K^{+}}\right. & & (a \in \mathcal{M}) \\
\widetilde{\Delta}_{\varphi}^{i t} \otimes \lambda(t) & =K\left(\left(\Delta_{\varphi}^{i t} \otimes \lambda(t)\right) \otimes_{\mathscr{L}_{L}} 1\right) K^{+} & & (t \in \mathbf{R}) \\
(u \otimes \lambda)(f) \otimes 1 & =K(W \otimes \lambda)(f) K^{+} & & \left(f \in \mathfrak{B}_{I}\right) .
\end{aligned}
$$

Thus it follows from Lemma 5.1 and Lemma 5.4 that $\mathrm{Ad} K$ gives an isomorphism between the algebras $\mathscr{P}$ and 2 .

Q.E.D.

Let $\theta$ be the dual action $\widehat{\sigma}^{\varphi}$ of the modular automorphism $\sigma^{\varphi}$. We decompose $\theta$ into

$$
\theta=\int_{X}^{\oplus} \theta^{x} d \mu(x)
$$

along the direct integral $\mathcal{M} \times{ }_{\sigma^{4}} \mathbf{R}=\int_{X}^{\oplus} \mathcal{M}(x) \times{ }_{\sigma^{4}{ }_{1}} \mathbf{R} d \mu(x)$. Then $\theta^{x}$ is the dual action of $\sigma^{\varphi_{1}}$ for $\mu$-a.e. $x \in X$. Note that, in view of (5.2) and (5.3), we have

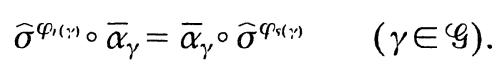

Accordingly, we get

$$
\theta^{r(\gamma) \circ \bar{\alpha}_{\gamma}}=\bar{\alpha}_{\gamma} \circ \theta^{s(\gamma)}
$$

for $v$-a.e. $\gamma \in \mathscr{G}$ and any $t \in \mathbf{R}$. It follows that the dual action $\theta$ leaves $\left(M \times{ }_{\sigma^{4}} \mathbf{R}\right)^{\bar{\alpha}}$ as well as $\mathscr{L}\left(\mathcal{M} \times{ }_{\sigma^{\prime}} \mathbf{R}\right)^{\bar{\alpha}}$ globally invariant.

Now we are in a position to state the main theorem in this section.

Theorem 5.11 (The smooth flow of weights of a crossed product). Let ( $\mathscr{G}$, $\left.\{M(x)\},\left\{\alpha_{\gamma}\right\}\right)$ and $\varphi$ be as before. Assume that $\mathscr{G}$ is principal. Then, as covariant systems, we have

$$
\left\{\mathscr{E}\left(\left(\mathcal{M} \times{ }_{a} \mathscr{G}\right) \times{ }_{\sigma^{\widetilde{4}}} \mathbf{R}\right), \widehat{\sigma}^{\widetilde{\varphi}}, \mathbf{R}\right\} \cong\left\{\mathscr{L}\left(\mathcal{M} \times_{\sigma^{4}} \mathbf{R}\right)^{\bar{\alpha}}, \theta, \mathbf{R}\right\} .
$$


Proof. Remark first that, because of the above observation, the covariant system $\left\{\mathscr{L}\left(\mathcal{M} \times_{\sigma^{4}} \mathbf{R}\right)^{\bar{\alpha}}, \theta, \mathbf{R}\right\}$ makes sense. Let $\mathscr{P}$ and $\mathscr{2}$ be as in Proposition 5.5. Then, due to the same proposition, $\mathscr{L}(\mathscr{P})$ is spatially isomorphic to $\mathscr{L}(\mathscr{2})$. Since $\mathscr{G}$ is principal, it follows from Theorem 3.2 (iii) that $\mathscr{L}(\mathscr{2})$ is isomorphic to $\mathscr{L}\left(M \times{ }_{\sigma^{\prime}} \mathbf{R}\right)^{\bar{\alpha}}$. Thanks to (5.6) and (5.7), it is easily verified that

$$
\begin{aligned}
K\left(\theta_{\mathrm{s}}(a \otimes 1) \otimes_{\mathscr{Z}} 1\right) K^{+} & =\widehat{\sigma}_{s}^{\widetilde{\varphi}}\left(\left(a \otimes_{\mathscr{L}} 1\right) \otimes 1\right) & & (a \in \mathcal{M}) \\
K\left(\theta_{s}\left(\Delta_{\varphi}^{i t} \otimes \lambda(t)\right) \otimes_{\mathscr{L}} 1\right) K^{+} & =\widehat{\sigma}_{s}^{\widetilde{\varphi}}\left(\widetilde{\Delta}_{\varphi}^{i t} \otimes \lambda(t)\right) & & (t \in \mathbf{R}) .
\end{aligned}
$$

This proves our assertion.

Q.E.D.

\section{§6. Examples of Groupoid Actions and Types of Their Crossed Products}

In this section, we give a few examples of actions of measured principal groupoids. Then we apply the results established so far to those examples in order to obtain some information on their crossed products. The examples will be constructed in the following manner. First we exhibit a homomorphism from a given principal groupoid into the normalizer of an (ergodic) countable group of automorphisms on a measure space. Secondly, we lift the homomorphism to the group of automorphisms of the von Neumann algebra derived from the ergodic transformations. Thus we obtain an action of a principal measured groupoid. In [Y1], we gave several interesting examples of groupoid actions. However we should say that the type of actions constructed below is new.

As we explained above, we are interested in this section mainly in groupoid actions $\left(\mathscr{G},\{M(x)\},\left\{a_{\gamma}\right\}\right)$ in which von Neumann algebras $\{M(x)\}_{\mathrm{r} \in X}$ do not vary in each fiber, that is, $\mathcal{M}(x)=\mathcal{M}_{0}$ for every $x \in X$. Thus we believe that it is convenient to rephrase Theorem 5.11 in this direction. For this, we need to introduce a notation. Suppose that $\mathcal{N}$ is a properly infinite von Neumann algebra, and that $\beta$ is an automorphism of $\mathcal{N}$. Let $\chi$ be a faithful normal semifinite weight on $\mathcal{N}$. Then, as in [H\&S], we may extend $\beta$ to an automorphism $\widetilde{\beta}$ of the crossed product $\mathcal{N} \times{ }_{\sigma} / \mathbb{R}$ in such a way that

$$
\begin{aligned}
\widetilde{\beta}(y \otimes 1) & =\beta(y) \otimes 1 \quad(y \in \mathcal{N}) \\
\widetilde{\beta}\left(\Delta_{\chi}^{i t} \otimes \lambda(t)\right) & =\left(D \chi \circ \beta^{-1}: D \chi\right)_{t} \Delta_{\chi}^{i t} \otimes \lambda(t) \quad(t \in \mathbb{R}) .
\end{aligned}
$$

It is shown in $[\mathrm{H} \& \mathrm{~S}]$ that the restriction of $\widetilde{\beta}$ to the center of the crossed product can be regarded as $\bmod \beta$, the image of $\beta$ under the fundamental homomorphism mod in the sense of Connes and Takesaki [C\&T]. (Note that Haagerup and Størmer proved this fact in [H\&S] in the case of $\mathcal{N}$ being a properly infinite factor. But their proof is still valid even if $\mathcal{N}$ is no longer a factor.) We now consider an action $\left(\mathscr{G},\left\{\mathcal{M}(x)=\mu_{0}\right\},\left\{\alpha_{\gamma}\right\}\right)$ of a principal measured groupoid $\mathscr{G}$. By Theorem 5.11, the smooth flow of weights of the corresponding crossed product is isomorphic to the flow $\left(\mathscr{L}\left(\mathcal{M} \times_{\sigma^{4}} \mathbb{R}\right)^{\bar{\alpha}}, \theta_{t}, \mathbb{R}\right)$, where $\varphi$ is a faithful normal state on $M$. In our case, $\mathcal{M}=\mathcal{M}_{0} \otimes L^{\infty}(X, \mu)$; so we may take $\varphi$ to be a 
state of the form $\varphi=\chi \otimes \tau$, where $\chi$ (resp. $\tau$ ) is a faithful normal state on $M_{0}$ (resp. $\left.L^{\propto}(X, \mu)\right)$. Then $\mathscr{L}\left(\mathcal{M} \times_{\sigma^{\sigma}} \mathbf{R}\right)=\mathscr{L}\left(\mathcal{M}_{0} \times{ }_{\sigma \times} \mathbf{R}\right) \otimes L^{\infty}(X, \mu)$. Let $\left(X_{M_{0}}\right.$, $\left.\mu_{M_{1}}, F_{t}^{M_{1}}\right)$ be a point realization of the smooth flow of weights $\left(\mathscr{E}\left(M_{0} \times{ }_{\sigma} \mathbf{R}\right)\right.$, $\left.\widehat{\sigma}_{t}^{\chi}, \mathbf{R}\right)$ of $\mathcal{M}_{0}$. Thus $\mathscr{L}\left(\mathcal{M} \times{ }_{\sigma^{4}} \mathbf{R}\right)$ can be viewed as $L^{x}\left(X_{\mu_{0}} \times X, \mu_{\mu_{0}} \times \mu\right)$. We now look at the induced action $\bar{\alpha}_{\gamma}$ of $\mathscr{G}$ discussed in the previous section. In our situation, each $\bar{\alpha}_{\gamma}$ is an automorphism of $\mu_{0} \times{ }_{\sigma \times} \mathbf{R}$. We assert that, with the notation introduced above,

$$
\bar{\alpha}_{\gamma}=\bar{\sigma}_{\log \delta\left(\gamma^{\prime}\right)}^{\chi} \widetilde{\alpha}_{\gamma} \quad(\gamma \in \mathscr{G}) .
$$

Indeed, by (5.2),

$$
\begin{aligned}
\bar{\alpha}_{\gamma}(a \otimes 1) & =\alpha_{\gamma}(a) \otimes 1 \\
& =\widehat{\sigma}_{\log \delta(\gamma)}^{\chi}\left(\alpha_{\gamma}(a) \otimes 1\right) \\
& =\hat{\sigma}_{\log \delta(\gamma)}^{\chi}{ }^{\circ} \widetilde{\alpha}_{\gamma}(a \otimes 1)
\end{aligned}
$$

for any $a \in M_{0}$. By (5.3), for any $t \in \mathbf{R}$, we have

$$
\begin{aligned}
\bar{\alpha}_{\gamma}\left(\Delta_{\chi}^{i t} \otimes \lambda(t)\right) & =\delta(\gamma)^{-l t}\left(D \chi \circ \alpha_{\gamma}^{-1}: D \chi\right)_{t} \Delta_{\chi}^{i t} \otimes \lambda(t) \\
& =\left(\left(D \chi \circ \alpha_{\gamma}^{-1}: D \chi\right)_{t} \otimes 1\right) \widehat{\sigma}_{\log \delta(\gamma)}^{\chi}\left(\Delta_{\chi}^{i t} \otimes \lambda(t)\right) \\
& =\widehat{\sigma}_{\log \delta(\gamma)}^{\chi}\left(\left(D \chi \circ \alpha_{\gamma}^{-1}: D \chi\right)_{t} \Delta_{\chi}^{i t} \otimes \lambda(t)\right) \\
& =\widehat{\sigma}_{\log \delta\left(\gamma^{\prime}\right)}^{\chi}{ }^{\circ} \widetilde{\alpha}_{\gamma}\left(\Delta_{\chi}^{i t} \otimes \lambda(t)\right) .
\end{aligned}
$$

Thus we get the identity (*). From $(*)$, it follows that, upon identifying $\mathscr{L}\left(\mathcal{M} \times_{\sigma^{4}} \mathbf{R}\right)$ with $L^{x}\left(X_{\mu_{0}} \times X, \mu_{\mathcal{M}_{0}} \times \mu\right), \mathscr{L}\left(\mathcal{M} \times{ }_{\sigma^{4}} \mathbf{R}\right)^{\bar{\alpha}}$ is a von Neumann subalgebra $P\left(\mu_{0}, \alpha\right)$ of $L^{\infty}\left(X_{\mu_{0}} \times X, \mu_{M_{0}} \times \mu\right)$ consisting of functions $f$ on $X_{\mu_{0}} \times X$ with the following property:

$$
f(\eta, r(\gamma))=f\left(F_{\log \delta(\gamma)}^{M_{1}}{ }^{\circ}(\bmod \alpha) \eta, s(\gamma)\right)
$$

for any $\eta \in X_{\mu_{0}}$ and $\gamma \in \mathscr{G}$. Thus the smooth flow of weights of the crossed product $\mathcal{M} \times{ }_{\alpha} \mathscr{G}$ is $\left(P\left(\mathcal{M}_{0}, \alpha\right), F_{t}^{M_{0}} \times i d, \mathbf{R}\right)$.

Let $\mathscr{G}$ be a principal measured groupoid. In this section too, we keep the notations introduced so far. Let us take a not necessarily ergodic countable group $G$ of automorphisms (i.e. nonsingular transformations) on a Lebesgue space $(\Omega, m)$. Then we choose a measurable homomorphism $\gamma \mapsto T_{\gamma}$ from $\mathscr{G}$ into the group $A(\Omega)$ of all automorphisms on $(\Omega, m)$ such that $T_{\gamma} \in N[G]$, the normalizer $\left\{T \in A(\Omega): T G T^{-1}=G\right\}$ of $G$, for all $\gamma \in \mathscr{G}$, where $A(\Omega)$ is equipped with the Borel structure induced by the topology described in $[\mathrm{H} \& \mathrm{O}]$. We denote by $\mu_{0}$ the von Neumann algebra constructed from the transformation $(\Omega, G, m)$ by the Krieger's construction. Since $T_{\gamma} \in N[G]$, each $T_{\gamma}$ extends to an automorphism $\alpha_{\gamma}$ of $M_{0}$. It is an easy exercise to verify that the system ( $\mathscr{G}$, $\left.\left\{M(x)=M_{0}\right\}_{x \in X},\left\{\alpha_{\gamma}\right\}_{\gamma \in \mathscr{G}}\right)$ is an action of $\mathscr{G}$. Let us now exhibit below two examples of groupoid actions of the above type. 
Example 6.1. Let $\mathscr{G}$ be a principal measured groupoid associated with a strictly aperiodic automorphism $T$ on a Lebesgue space $(X, \mu)$. Namely,

$$
\mathscr{G}=\left\{\left(x, T^{k} x\right): x \in X, k \in \mathbb{Z}\right\} .
$$

By "strictly aperiodic", we mean that the transformation $T$ admits no periodic points. We take a (measurable) homomorphism $t(\cdot)$ from $\mathscr{G}$ into the set $\mathbf{R}$ of real numbers. (Such a homomorphism can be easily constructed. For example, let us take a measurable real-valued function $f$. Then define $t(\cdot)$ by

$$
t(x, y)= \begin{cases}\sum_{i=0}^{k-1} f\left(T^{i} x\right) & \text { if } y=T^{k} x \quad(k>0) ; \\ 0 & \text { if } y=x ; \\ -\sum_{i=0}^{k-1} f\left(T^{i} y\right) & \text { if } y=T^{-k} x \quad(k>0) .\end{cases}
$$

Obviously, $t(\cdot)$ is a measurable homomorphism from $\mathscr{G}$ into $\mathbb{R}$. Note that $t(\cdot)$ is nontrivial unless $f \equiv 0$.) We now make use of an example given by Hamachi in [Hm]. Let $R$ be an ergodic automorphism of type $I I I_{1}$ on a Lebesgue space ( $Y$, $\left.\mu_{Y}\right)$, and $\left\{F_{t}\right\}_{t \in \mathbf{R}}$ be measurable ergodic flow of automorphisms on a measure space $\left(Z, \mu_{Z}\right)$. We put $\Omega=Y \times Z \times \mathbf{R}, m=\mu_{Y} \times \mu_{Z} \times e^{u} d u$, where $d u$ is the Lebesgue measure on $\mathbf{R}$. Define automorphisms $\widetilde{R}$ and $\left\{\widetilde{F}_{t}\right\}_{t \in \mathbb{R}}$ on $\Omega$ by

$$
\begin{aligned}
& \widetilde{R}(y, z, u)=\left(R y, z, u-\log \frac{d \mu_{Y} \circ R}{d \mu_{Y}}(y)\right) \\
& \widetilde{F}_{t}(y, z, u)=\left(y, F_{t} z, u+t-\log \frac{d \mu_{Z} \circ F_{t}}{d \mu_{Z}}(z)\right)
\end{aligned}
$$

for $t \in \mathbb{R}$ and $(y, z, u) \in \Omega$. We denote by $G$ the countable abelian group generated by automorphisms $\widetilde{R}$ and $\left\{\widetilde{F}_{t}\right\}_{t \in \Gamma}$, where $\Gamma$ is a countable dense subgroup of $\mathbb{R}$. Clearly, $G$ is ergodic. It is shown in $[\mathrm{Hm}]$ that the Krieger factor $\mathcal{M}_{0}$ coming from this system $(G, \Omega, m)$ is a factor of type $I I I$ whose smooth flow of weights is isomorphic to the given flow $\left\{F_{t}\right\}$. For each $\gamma \in \mathscr{G}$, define an automorphism $T_{\gamma}$ on $\Omega$ by

$$
T_{\gamma}(y, z, u)=(y, z, u-t(\gamma))
$$

It is easy to check that $T_{\gamma}$ commutes with elements in $G$; so $T_{\gamma} \in N[G]$.

Example 6.2. Let $\mathscr{G}$ and $t(\cdot)$ be as in Example 6.1, but we assume here $t(\cdot)$ to be integer-valued. This time, we make use of an example due to Krieger (see [H\&O]). Suppose that $G_{0}$ is a type $I I I_{\lambda}$ ergodic countable group of automorphisms on a Lebesgue space $\left(Y, \mu_{Y}\right)$, where $\mu_{Y}$ is an admissible measure (see [H\&O] for the definition of "an admissible measure"), and that $U$ is an ergodic $\mu_{Z}$-preserving transformation on a Lebesgue space $\left(Z, \mu_{Z}\right)$. Put $\Omega=Y \times Z$, $m=\mu_{Y} \times \mu_{Z}$. For each $g \in G_{0}$, define an automorphism $\widetilde{g}$ on $\Omega$ by 


$$
\widetilde{g}(y, z)=\left(g y, U^{n(g, y)} z\right)
$$

for $(y, z) \in \Omega$, where $n(g, y)$ is an integer determined by $d\left(\mu_{Y} \circ g\right) / d \mu_{Y}(y)=$ $\lambda^{n(g, y)}$ for $\mu_{Y}$-a.e. $y \in Y$ and $g \in G_{0}$. Set $G=\left\{\widetilde{g}: g \in G_{0}\right\}$. $G$ is a type $I I I_{0}$ ergodic countable group of transformations on $(\Omega, m)$. For each $\gamma \in \mathscr{G}$, define $T_{\gamma}$ on $\Omega$ by

$$
T_{\gamma}(y, z)=\left(y, U^{r(\gamma)} z\right)
$$

Obviously, $T_{\gamma}$ commutes with $\widetilde{g}\left(g \in G_{0}\right)$; so $T_{\gamma} \in N[G]$ for all $\gamma \in \mathscr{G}$.

Thus we are able to construct many interesting examples of groupoid actions that possess the required property. Now we return to our original (abstract) system $\left(G,\left\{\mathcal{M}(x)=\mathcal{M}_{0}\right\},\left\{\alpha_{\gamma}\right\}\right)$. We denote by $R_{\Omega}$ the graph of the equivalence relation generated by $(G, \Omega, m)$. Then $M_{0}$ is acting on a Hilbert space $L^{2}\left(R_{\Omega}\right)$, constructed via Feldman and Moore's method [F\&M2]. Let $\mathcal{M}=\int_{X}^{\oplus} \mathcal{M}(x) d \mu(x)$ $=M_{0} \otimes L^{x}(X, \mu)$. This acts on $\mathscr{H}=L^{2}\left(R_{\Omega}\right) \otimes L^{2}(X, \mu)$. We denote by $\omega$ the faithful normal state on $M_{0}$ determined by the characteristic function of the diagonal set of $R_{\Omega}$. (For this, we should choose a probability measure on $\Omega$ equivalent to $m)$. The crossed product $M \times{ }_{a} \mathscr{G}$ by this action is represented on a Hilbert space $\mathscr{H} \otimes_{\mu \mathscr{x}} L^{2}(\mathscr{G}, v)=L^{2}\left(R_{\Omega}\right) \otimes_{\mathscr{X}} L^{2}(\mathscr{G}, v)$.

Before we proceed, we prepare three lemmas.

Lemma 6.3. Let $\mathscr{G}$ be a principal measured groupoid as before. Let $(\mathscr{G}$, $\left.\left\{\mathscr{P}(x)=\mathscr{P}_{0}\right\},\left\{\beta_{\gamma}\right\}\right)$ be an action of $\mathscr{G}$, where $\mathscr{P}_{0}$ is a fixed factor with separable predual. Put $\mathscr{P}=\int_{X}^{\oplus} \mathscr{P}(x) d \mu(x)=\mathscr{P}_{0} \otimes L^{\infty}(X, \mu)$. Then the center $\mathscr{E}\left(\mathscr{P} \times{ }_{\beta} \mathscr{G}\right)$ of the crossed product is isomorphic to the algebra $\mathfrak{T}_{0}=\left\{f \in L^{\infty}(X, \mu): f(s(\gamma))=\right.$ $f(r(\gamma)) v$-a.e. $\gamma \in \mathscr{G}\}$. Thus, if $G$ is ergodic, then the crossed product is a factor.

Proof. By Theorem 3.2 (iii), the center $\mathscr{L}\left(\mathscr{P} \times{ }_{\beta} \mathscr{G}\right)$ is isomorphic to $\mathscr{L}(\mathscr{P})^{\beta}$. Since $\mathscr{L}(\mathscr{P})=\mathscr{L}\left(\mathscr{P}_{0} \otimes L^{\infty}(X, \mu)\right)=\mathbf{C} \otimes L^{\infty}(X, \mu), \mathscr{L}(\mathscr{P})^{\beta}$ coincides with the algebra $\mathfrak{Y K}_{0}$. Thus ergodicity of $\mathscr{G}$ implies that the crossed product is a factor.

Q.E.D.

Lemma 6.4. Let $\left(\mathscr{G},\left\{\mathscr{P}(x)=\mathscr{P}_{0}\right\},\left\{\beta_{\gamma}\right\}\right)$ be as in Lemma 6.3. If $\mathscr{P}_{0}$ is of type III, then so is the crossed product $\mathscr{P} \times{ }_{\beta} \mathscr{G}$.

Proof. Note that, since $\mathscr{P}=\mathscr{P}_{0} \otimes L^{x}(X, \mu)$, we have that $T(\mathscr{P})=T\left(\mathscr{P}_{0}\right)$. Suppose that $\mathscr{P} \times{ }_{\beta} \mathscr{G}$ were semifinite. Then, by [T1] or [P\&T], $T\left(\mathscr{P} \times{ }_{\beta} \mathscr{G}\right)=\mathbf{R}$. Due to Theorem $4.2, T\left(\mathscr{P} \times{ }_{\beta} \mathscr{G}\right)$ is a subset of $T(\mathscr{P})=T\left(\mathscr{P}_{0}\right)$; so $T\left(\mathscr{P}_{0}\right)$ must be the whole real number group $\mathbf{R}$. Thus $\sigma_{t}^{\chi} \in \operatorname{Int}\left(\mathscr{P}_{0}\right)$ for all $t \in \mathbf{R}$ and any faithful normal state $\chi$ on $\mathscr{P}_{0}$. Since $\mathscr{P}_{0}$ is a factor with separable predual, it follows from [T1] that, upon fixing some faithful normal state $\chi$, there exists a (continuous) one-parameter unitary group $\{u(t)\}_{t \in \mathbf{R}}$ in $\mathscr{P}_{0}$ such that $\sigma_{t}^{\chi}=\operatorname{Ad} u(t)(t \in \mathbf{R})$. 
From [T1] or $[\mathrm{P} \& \mathrm{~T}], \mathscr{P}_{0}$ must be semifinite, which is a contradiction. Q.E.D.

Lemma 6.5. Let $\left(\mathscr{G},\left\{\mathscr{P}(x)=\mathscr{P}_{0}\right\},\left\{\beta_{\gamma}\right\}\right)$ be as in Lemma 6.3. If $\mathscr{G}$ is ergodic and $\mathscr{P}_{0}$ is a separable factor of type $\mathrm{III}_{1}$, then so is the crossed product $\mathscr{P} \times{ }_{\beta} \mathscr{G}$.

Proof. By Lemmas 6.3 and 6.4, the crossed product $\mathscr{P} \times{ }_{\beta} \mathscr{G}$ is a factor of type III. Let $\chi$ be as in the proof of Lemma 6.4, and $\tau$ be a faithful normal state on $L^{x}(X, \mu)$. Put $\varphi_{0}=\chi \otimes \tau$. From Theorem 5.11, we have that $\mathscr{L}\left(\left(\mathscr{P} \times{ }_{\beta} \mathscr{G}\right) \times{ }_{\sigma_{\pi_{0}}} \mathbf{R}\right) \cong \mathscr{L}\left(\mathscr{P} \times{ }_{\sigma_{\psi_{0}}} \mathbf{R}\right)^{\bar{\beta}}$. Note that, by our assumption, $\mathscr{L}\left(\mathscr{P} \times{ }_{\sigma_{\psi_{0}}} \mathbf{R}\right)$ is equal to $\mathscr{L}\left(\mathscr{P}_{0} \times{ }_{\sigma}, \mathbf{R}\right) \otimes L^{x}(X, \mu)=\mathbf{C} \otimes L^{x}(X, \mu)$. Thus $\mathscr{L}\left(\mathscr{P} \times{ }_{\sigma^{\alpha_{0}}} \mathbb{R}\right)^{\widetilde{\beta}}$ is isomorphic to the algebra $\mathfrak{Y}_{0}$ in Lemma 6.3, which reduces to $\mathbb{C}$ in this case. Hence $\mathscr{L}\left(\left(\mathscr{P} \times{ }_{\beta} \mathscr{G}\right) \times{ }_{\sigma \widetilde{\pi}_{1}} \mathbf{R}\right)=\mathbb{C}$. Therefore $\mathscr{P} \times{ }_{\beta} \mathscr{G}$ is of type $\mathrm{III}_{1}$.

Q.E.D.

Lemma 6.6. Let $\left(\mathscr{G},\left\{\mathscr{P}(x)=\mathscr{P}_{0}\right\},\left\{\beta_{\gamma}\right\}\right)$ be as in Lemma 6.3 again. Suppose that $\beta_{\gamma} \in \operatorname{Int}\left(\mathscr{P}_{0}\right)$ for all $\gamma \in \mathscr{G}$. Then

$$
\mathscr{P} \times{ }_{\beta} \mathscr{G}=\mathscr{P}_{0} \otimes \Re(\mathscr{G}) .
$$

Proof. Let $\mathscr{H}_{0}=L^{2}\left(\mathscr{P}_{0}\right)$ and $\mathscr{H}=\int_{X}^{\oplus} \mathscr{H}_{0} d \mu(x)=\mathscr{H}_{0} \otimes L^{2}(X, \mu)$. Then, by definition, the crossed product $\mathscr{P} \times{ }_{\beta} \mathscr{G}$ acts on $\mathscr{H}_{\mu} \otimes_{\mu \mathscr{L}} L^{2}(\mathscr{G}, \mu)=\mathscr{H}_{0} \otimes_{\mathscr{q}_{\mathcal{L}}} L^{2}(\mathscr{G}, v)$. Note that, since $\beta_{\gamma} \in \operatorname{Int}\left(\mathscr{P}_{0}\right)$, the canonical implementation $u(\gamma)$ of $\beta_{\gamma}$ on $L^{2}\left(\mathscr{P}_{0}\right)$ belongs to $\mathscr{P}_{0}$. For each $f \in \mathfrak{B}_{I}$, define $u_{f}$ by

$$
u_{f}(\gamma)=f(\gamma) u(\gamma)^{+} \quad(\gamma \in \mathscr{G}) \text {. }
$$

Then $u_{f} \in \mathscr{F}_{\varphi_{0}}$, where $\varphi_{0}=\chi \otimes \tau$ is the state on $\mathscr{P}$ that appeared in the previous lemma. It can be shown that

$$
\Phi\left(A_{u_{f}}\right)=1_{\mathscr{H}_{0}} \otimes \lambda(f),
$$

where $\lambda$ denotes the integrated representation of the left regular representation of $\mathscr{G}$. Note that $\left\{a \otimes_{\mathscr{L}} 1: a \in \mathscr{P}\right\}$ coincides with $\mathscr{P}_{0} \otimes \mathscr{L}_{R}$. Thus, due to Theorem $2.14, \mathscr{P}_{0} \otimes \mathscr{R}(\mathscr{G})$ is contained in $\mathscr{P} \times{ }_{\beta} \mathscr{G}$. Hence we have $\left(\mathscr{P} \times{ }_{\beta} \mathscr{G}\right)^{\prime} \subseteq \mathscr{P}_{0}^{\prime} \otimes \mathscr{R}(\mathscr{G})^{\prime}$. For the reverse inclusion, consider a function $c(\cdot)$ given by

$$
c(\gamma)=f(\gamma) b \quad(\gamma \in \mathscr{G}) \text {, }
$$

where $f \in \mathfrak{S}_{I}$ and $b \in \mathscr{P}_{0}$. Then $c(\cdot) \in \mathscr{F}_{\varphi_{0}}$. It is easy to check that we have

$$
\widetilde{J}_{\varphi_{1}} \Phi\left(A_{c}\right) \widetilde{J}_{\varphi_{10}}=J_{\chi} b J_{\chi} \otimes \rho(J \bar{f}) .
$$

It follows that $\mathscr{P}_{0}^{\prime} \otimes \mathscr{R}(\mathscr{G})^{\prime} \subseteq\left(\mathscr{P} \times{ }_{\beta} G\right)^{\prime}$.

Q.E.D.

Let us now look at Example 6.1. In $[\mathrm{Hm}]$, Hamachi showed that the associated flow of $(G, \Omega, m)$ in Example 6.1 is exactly the given flow $\left\{F_{t}\right\}_{t \in \mathbb{R}}$. Hence, if we take $\left(\left\{F_{t}\right\}, Z, \mu_{Z}\right)$ to be a trivial flow, that is, $Z=\{$ point $\}$, then $G$ is a type III $I_{1}$ transformation group, so that $M_{0}$ is a factor of type III $I_{1}$. Therefore, 
by Lemma 6.5 , the crossed product $M \times{ }_{\alpha} \mathscr{G}$ is a factor of type $I I I_{1}$, provided that $T$ is an ergodic transformation. We can argue in a different way to conclude that the crossed product is a factor of type $I I I_{1}$ in the above situation. In fact, if the flow space $Z$ is trivial, then it is easy to see that the action $T_{\gamma}$ falls not only in $N[G]$ but also in $[G]$, the full group of $G$. It follows that the action $\alpha_{\gamma}$ on $\mu_{0}$ induced by $T_{\gamma}$ is an inner action. Hence, by Lemma 6.6 , the crossed product $M \times{ }_{\alpha} \mathscr{G}$ is $M_{0} \otimes \mathscr{R}(\mathscr{G})$. Since $M_{0}$ is of type $I I I_{1}$, so is the crossed product.

We return to the original system $\left(\mathscr{G},\left\{M(x)=\mathcal{M}_{0}\right\},\left\{\alpha_{\gamma}\right\}\right)$. Our next objective is to clarify the structure of the crossed product $M \times{ }_{a} G$ by the above action. It turns out that the crossed product can be realized as the von Neumann algebra constructed from an equivalence relation on a measure space. For this purpose, we introduce an equivalence relation $R_{\Omega \times X}$ on $\Omega \times X$ by saying that $(\omega, x)$ is equivalent to $\left(\omega^{\prime}, x^{\prime}\right)$ if $x \sim x^{\prime}$ in $X$ and $\omega \sim T_{\left(x, x^{\prime}\right)} \omega^{\prime}$ in $\Omega$. It follows, from the fact that $T_{\gamma} \in N[G]$ for all $\gamma \in \mathscr{G}$, that the notion introduced above is indeed an equivalence relation on $\Omega \times X$. The groupoid $R_{\Omega \times X}$ has a canonical standard measured groupoid structure as follows. For $(\omega, x) \in \Omega \times X$, a Haar system $v^{(\omega, x)}$ is given by

$$
v^{(\omega, x)}(f)=\int \sum_{\omega^{\prime} \sim \omega} f\left((\omega, x),\left(T_{\gamma^{-1}} \omega^{\prime}, s(\gamma)\right) d \lambda^{2}(\gamma)\right.
$$

where $f$ is a positive Borel function on $R_{\Omega \times X}$ and $\Sigma_{\omega^{\prime} \sim \omega}$ indicates a sum with respect to $\omega^{\prime}$ equivalent to a given $\omega$. A quasi-invariant measure on $\Omega \times X$ is the product measure $m \times \mu$ with module $\delta(\cdot) D(\cdot, \cdot)$, where $D(\cdot, \cdot)$ is the module corresponding to the measured groupoid $R_{\Omega}$. Namely, if we define a measure $\theta$ on $R_{\Omega \times X}$ (This $\theta$ has nothing to do with the one that appeared in $\S 6$.) by

$$
\theta(f)=\int_{\Omega \times X} \int \sum_{\omega^{\prime} \sim \omega} f\left((\omega, x),\left(T_{\gamma^{-1}} \omega^{\prime}, s(\gamma)\right) d \lambda^{x}(\gamma) d(m \times \mu)(\omega, x)\right.
$$

and $\theta^{-1}$ by the image of $\theta$ under the inverse map of $R_{\Omega \times X}$, then

$$
\frac{d \theta}{d \theta^{-1}}\left((\omega, r(\gamma)),\left(\omega^{\prime}, s(\gamma)\right)\right)=\delta(\gamma) D\left(\omega, T_{\gamma} \omega^{\prime}\right)
$$

Recall that, as we noted just before Lemma 6.3, the crossed product $M \times{ }_{a} G$ acts on $L^{2}\left(R_{\Omega}\right) \otimes{ }_{*} L^{2}(G, v)$. Our next step is to construct a unitary that carries $L^{2}\left(R_{\Omega}\right) \otimes_{\Psi^{2}} L^{2}(\xi, v)$ onto $L^{2}\left(R_{\Omega \times X}, \theta\right)$. For this, let $\zeta \in L^{2}\left(R_{\Omega}\right) \otimes_{x_{x}} L^{2}(G, v)$. Then define a function $U \xi$ on $R_{\Omega \times X}$ by

$$
\{U \zeta\}\left((\omega, r(\gamma)),\left(\omega^{\prime}, s(\gamma)\right)\right)=\zeta\left(\left(\omega, T_{\gamma} \omega^{\prime}\right), \gamma\right)
$$

Then

$$
\begin{aligned}
\theta\left(|U \zeta|^{2}\right) & =\int_{\Omega \times X} \int \sum_{\omega^{\prime} \sim \omega}\left|\{U \zeta\}\left((\omega, x),\left(T_{\gamma^{-1}} \omega^{\prime}, s(\gamma)\right)\right)\right|^{2} d \lambda^{x}(\gamma) d(m \times \mu)(\omega, x) \\
& =\int_{\Omega} \int_{X} \int_{\omega^{\prime} \sim \omega}\left|\zeta\left(\left(\omega, \omega^{\prime}\right), \gamma\right)\right|^{2} d \lambda^{x}(\gamma) d \mu(x) d m(\omega) \\
& =\|\zeta\|_{2}^{2} .
\end{aligned}
$$


Thus $U$ is an isometry from $L^{2}\left(R_{\Omega}\right) \otimes_{\mathscr{L}} L^{2}(\mathscr{G}, v)$ into $L^{2}\left(R_{\Omega \times X}, \theta\right)$. Let $f \in$ $L^{2}\left(R_{\Omega \times X}, \theta\right)$. This time, we define a function $V f$ on $R_{\Omega} \times \mathscr{G}$ by

$$
\{V f\}\left(\left(\omega, \omega^{\prime}\right), \gamma\right)=f\left((\omega, r(\gamma)),\left(T_{\gamma^{-1}} \omega^{\prime}, s(\gamma)\right)\right)
$$

Then we have

$$
\begin{aligned}
& \int_{\Omega} \int_{X} \int_{\omega^{\prime} \sim \omega}\left|\{V f\}\left(\left(\omega, \omega^{\prime}\right), \gamma\right)\right|^{2} d \lambda^{x}(\gamma) d \mu(x) d m(\omega) \\
= & \int_{\Omega} \int_{X} \int_{\omega^{\prime} \sim \omega}\left|f\left((\omega, r(\gamma)),\left(T_{\gamma^{-1}} \omega^{\prime}, s(\gamma)\right)\right)\right|^{2} d \lambda^{x}(\gamma) d \mu(x) d m(\omega) \\
= & \|f\|^{2},
\end{aligned}
$$

which shows that $V$ is also an isometry from $L^{2}\left(R_{\Omega \times X}, \theta\right)$ into $L^{2}\left(R_{\Omega}\right) \otimes_{\mathscr{L}} L^{2}(\varphi, v)$. It is easy to see that $U V=1, V U=1$. Hence $U$ is a unitary with $U^{*}=V$.

What we would like to do next is to show that $\operatorname{Ad} U$ gives a spatial isomorphism between the crossed product $\mathcal{M} \times_{\alpha} \mathscr{G}$ and the von Neumann algebra $W^{*}\left(R_{\Omega \times X}\right)$ constructed from the principal measured groupoid $R_{\Omega \times X}$.

First we note that, by Corollary $2.5,\left(\mathcal{M} \times{ }_{\alpha} G\right)^{\prime}$ is generated by operators $\alpha^{\prime}\left(b^{\prime}\right)\left(b \in \mathcal{M}_{0}^{\prime}\right)$ and $1 \otimes \rho(f)\left(f \in \mathscr{B}_{l}\right)$. Moreover, the operators $\pi_{r}(k)$ $\left(k \in L^{\infty}(\Omega, m)\right)$ and $v(g)(g \in G)$ on $L^{2}\left(R_{\Omega}\right)$ given by

$$
\begin{aligned}
& \left\{\pi_{r}(k) p\right\}\left(\omega, \omega^{\prime}\right)=k\left(\omega^{\prime}\right) p\left(\omega, \omega^{\prime}\right) \quad\left(p \in L^{2}\left(R_{\Omega}\right)\right) \\
& \{v(g) p\}\left(\omega, \omega^{\prime}\right)=\left[\frac{d m \circ g^{-1}}{d m}\left(\omega^{\prime}\right) D\left(\omega^{\prime}, g^{-1} \omega^{\prime}\right)\right]^{1 / 2} p\left(\omega, g^{-1} \omega^{\prime}\right)
\end{aligned}
$$

are generators of $\mu_{0}$. Consequently, $\left(\mathcal{M} \times{ }_{\alpha} G\right)^{\prime}$ is generated by operators of the forms $\alpha^{\prime}\left(\pi_{r}(k)\right), \alpha^{\prime}(v(g))$ and $1 \otimes \rho(f)$. Similarly, $\mathcal{M} \times{ }_{\alpha} \varphi$ is engendered by operators of the forms $\pi_{\ell}(k) \otimes 1\left(k \in L^{x}(\Omega, m)\right), w(g) \otimes 1(g \in G)$ and $(u \otimes \lambda)(f)\left(f \dot{\in} \mathfrak{B}_{I}\right)$, where $\pi_{\ell}(k)$ and $w(g)$ are operators generating $M_{0}$ defined by

$$
\begin{aligned}
& \left\{\pi_{\ell}(k) p\right\}\left(\omega, \omega^{\prime}\right)=k(\omega) p\left(\omega, \omega^{\prime}\right) \\
& \{w(g) p\}\left(\omega, \omega^{\prime}\right)=\left[\frac{d m \circ g^{-1}}{d m}(\omega)\right]^{1 / 2} p\left(g^{-1} \omega, \omega^{\prime}\right) .
\end{aligned}
$$

Let $\mathfrak{B}_{I}\left(R_{\Omega \times X}\right)$ denote the " $R_{\Omega \times X}$-version of $\mathfrak{B}_{I}$ ". In other words, $\mathfrak{B}_{I}\left(R_{\Omega \times X}\right)$ is the \#-algebra $\mathcal{B}_{I}$ in $L^{2}\left(R_{\Omega \times X}, \theta\right)$ constructed by the procedure described in $\S 1$ from the measured groupoid $R_{\Omega \times X}$ instead of $\mathscr{G}$. From now on, we often write $\mathcal{E}_{I}$ for this set $\mathfrak{B}_{I}\left(R_{\Omega \times X}\right)$ for short, if there is no danger of confusion. We denote by $L(\cdot)$ (resp. $R(\cdot))$ the left (resp. right) multiplication of the Tomita algebra $\tilde{E}_{I}$, that is,

$$
\begin{aligned}
& L(f) g=f * g \quad\left(f, g \in \mathfrak{G}_{I}\right) \\
& R(f) g=g * f .
\end{aligned}
$$

Then operators of the form $L(f)\left(f \in \mathfrak{S}_{I}\right)$ (resp. $\left.R(f)\right)$ generate $W^{*}\left(R_{\Omega \times X}\right)$ (resp. $\left.W^{*}\left(R_{\Omega \times X}\right)^{\prime}\right)$. 
We first assert that $U\left(M \times{ }_{\alpha} \xi\right) U^{*} \subseteq W^{*}\left(R_{\Omega \times X}\right)$. For this, it suffices to show that operators of the types $U\left(\pi_{\ell}(k) \otimes 1\right) U^{*}, U(w(t) \otimes 1) U^{*}$ and $U(u \otimes \lambda)(h) U^{*}$ commute with $R(f)\left(f \in \tilde{S}_{l}\right)$. It is a tedious computation to check this fact. So we only mention below how those operators act on $L^{2}\left(R_{\Omega \times X}, \theta\right)$.

$$
\begin{aligned}
\left\{U\left(\pi_{\ell}(k) \otimes 1\right) U^{*} g\right\}\left((\omega, r(\gamma)),\left(\omega^{\prime}, s(\gamma)\right)\right)= & k(\omega) g\left((\omega, r(\gamma)),\left(\omega^{\prime}, s(\gamma)\right)\right) \\
\left\{U(w(t) \otimes 1) U^{*} g\right\}\left((\omega, r(\gamma)),\left(\omega^{\prime}, s(\gamma)\right)\right)= & {\left[\frac{d m^{\circ} t^{-1}}{d m}(\omega)\right]^{1 / 2} } \\
& g\left(\left(t^{-1} \omega, r(\gamma)\right),\left(\omega^{\prime}, s(\gamma)\right)\right) \\
\left\{U(u \otimes \lambda)(h) U^{*} g\right\}\left((\omega, r(\gamma)),\left(\omega^{\prime}, s(\gamma)\right)\right)= & \int h\left(\gamma_{1}\right) g\left(\left(T_{\gamma_{1}^{-1}} \omega, s\left(\gamma_{1}\right)\right),\right. \\
& \left.\left(\omega^{\prime}, s(\gamma)\right)\right) d \lambda^{r(\gamma)}\left(\gamma_{1}\right) .
\end{aligned}
$$

Next we claim that $U\left(M \times{ }_{\alpha} G\right)^{\prime} U^{*} \subseteq W^{*}\left(R_{\Omega \times X}\right)^{\prime}$. For this claim, we need only to prove that operators of the forms $U \alpha^{\prime}\left(\pi_{r}(k)\right) U^{*}, U \alpha^{\prime}(v(g)) U^{*}$ and $U(1 \otimes \rho(f)) U^{*}$ commute with $L(f)\left(f \in \mathfrak{S}_{I}\right)$. Again, we just state what the above operators do to vectors in $L^{2}\left(R_{\Omega \times X}, \theta\right)$.

$$
\begin{aligned}
\left\{U \alpha^{\prime}\left(\pi_{r}(k)\right) U^{*} g\right\}\left((\omega, r(\gamma)),\left(\omega^{\prime}, s(\gamma)\right)\right)= & k\left(\omega^{\prime}\right) g\left((\omega, r(\gamma)),\left(\omega^{\prime}, s(\gamma)\right)\right) \\
\left\{U \alpha^{\prime}(v(t)) U^{*} g\right\}\left((\omega, r(\gamma)),\left(\omega^{\prime}, s(\gamma)\right)\right)= & {\left[\frac{d m \circ t^{-1}}{d m}\left(\omega^{\prime}\right) D\left(\omega^{\prime}, t^{-1} \omega^{\prime}\right)\right]^{1 / 2} } \\
& g\left((\omega, r(\gamma)),\left(t^{-1} \omega^{\prime}, s(\gamma)\right)\right. \\
\left\{U(1 \otimes \rho(f)) U^{*} g\right\}\left((\omega, r(\gamma)),\left(\omega^{\prime}, s(\gamma)\right)\right)= & \int h\left(\gamma_{1}^{-1} \gamma\right) g((\omega, r(\gamma)), \\
& \left.\left(T_{\gamma_{1}^{-1} \gamma^{\prime}} \omega^{\prime}, s(\gamma)\right)\right) d \lambda^{r(\gamma)}\left(\gamma_{1}\right) .
\end{aligned}
$$

We now summarize the result established in the above discussion.

Theorem 6.7. Under the situation we have been considering so far, the crossed product $M \times{ }_{\alpha} \mathscr{G}$ is spatially isomorphic to the von Neumann algebra $W^{*}\left(R_{\Omega \times X}\right)$ derived from the measured equivalence relation $R_{\Omega \times X}$.

We consider the following nontrivial example of an action of an ergodic principal measured groupoid $\mathscr{G}$ of the above type.

Example 6.8. Suppose that $\theta_{1}$ and $\theta_{2}$ are rationally independent irrational real numbers. Put $\Omega=\mathbf{T}$, the set of complex numbers of radius 1 . We denote by $m$ the normalized Haar measure for $\mathbf{T}$. Then define an automorphism $S$ on $\Omega$ to be the irrational rotation by $\theta_{1}, S \omega=e^{2 \pi i \theta_{1}} \omega$. Let $M_{0}$ be the Krieger factor, which is the injective factor of type $I I_{1}$, obtained from this ergodic transformation. Next we set $(X, \mu)$ to be the infinite direct product measure space

$$
X=\prod_{n=1}^{\infty}\left(\{0,1\},\left\{\frac{1}{1+\lambda}, \frac{\lambda}{1+\lambda}\right\}\right)
$$


built from one measure space $\{0,1\}$ with $\left\{\frac{1}{1+\lambda}, \frac{\lambda}{1+\lambda}\right\}$ as its probability distribution, where $0<\lambda<1$. We introduce an equivalence relation on $X$ by declaring that $x=\left(x_{i}\right)_{i=1}^{\infty}$ in $X$ is equivalent to $x^{\prime}=\left(x_{i}^{\prime}\right)_{i=1}^{x}$ in $X$ if $x_{i}=x_{i}^{\prime}$ except for a finite number of $i$. It is well-known that this equivalence relation on $X$ is exactly the one that is generated by the so-called adding machine transformation on $X$. It follows that, if we denote by $\mathscr{G}$ the graph of this equivalence relation, then $\mathscr{G}$ is a type $I I I_{\lambda}$ ergodic orbitally discrete principal measured groupoid. Hence the module $\delta$ of $\varphi$ takes values of integer powers of $\lambda$. We put $n\left(x, x^{\prime}\right)=\log _{\lambda} \delta(x$, $\left.x^{\prime}\right) \in \mathbb{Z}$. Then, for any $\left(x, x^{\prime}\right) \in \mathscr{G}$, we define an automorphism $T_{\left(x, x^{\prime}\right)}$ on $\Omega$ by

$$
T_{\left(x, x^{\prime}\right)} \omega=e^{2 \pi i n\left(x, x^{\prime}\right) \theta_{2}} \omega \quad(\omega \in \Omega) .
$$

It is easy to check that $S \circ T_{\left(x, x^{\prime}\right)}=T_{\left(x, x^{\prime}\right)} \circ S$ for any $\left(x, x^{\prime}\right) \in \mathscr{G}$. Thus $T_{\left(x, 1^{\prime}\right)}$ belongs to $N[S]$. Moreover, it can be readily shown that $T_{\left(x, x^{\prime}\right)} \in N[S] \backslash[S]$ unless $x=x^{\prime}$. Accordingly, every $T_{\left(x, x^{\prime}\right)}\left(x \neq x^{\prime}\right)$ induces an outer automorphism $\alpha_{\left(x, x^{\prime}\right)}$ of $\mathcal{M}_{0}$. Thus we obtain a nontrivial action $\left(\mathscr{G},\left\{\mathcal{M}(x)=\mathcal{M}_{0}\right\},\left\{\alpha_{\left(\mathrm{r}, x^{\prime}\right)}\right\}\right)$ of an ergodic principal measured groupoid $\mathscr{G}$.

In this paragraph, we would like to apply Theorem 6.7 to the above action in order to analyze the type of its crossed product algebra. By Theorem 6.7, the crossed product by this action is isomorphic to the algebra $W^{*}\left(R_{\Omega \times X}\right)$. Hence we need to compute the Poincaré flow of the equivalence relation $R_{\Omega \times X}$ so as to determine the type of the factor $W^{*}\left(R_{\Omega \times X}\right)$. For the computation, we look at a measure space $\left(\Omega \times X \times \mathbb{R}, m \times \mu \times e^{u} d u\right)$, where $d u$ is, as usual, the Lebesgue measure on $\mathbb{R}$. On this measure space, we consider an equivalence relation in which $(\omega, x, u)$ is equivalent to $\left(\omega^{\prime}, x^{\prime}, u^{\prime}\right)$ if $(\omega, x) \sim\left(\omega^{\prime}, x^{\prime}\right)$ in $R_{\Omega \times X}$ and $u=u^{\prime}+\log \delta\left(x, x^{\prime}\right)$. We need to decompose $\Omega \times X \times \mathbb{R}$ into ergodic components with respect to the above equivalence relation and examine the behavior of the flow $\left\{F_{t}\right\}_{t \in \mathbb{R}}$ on $\Omega \times X \times \mathbb{R}$ according to the decomposition, where $F_{t}$ is given by $F_{t}(\omega, x, u)=(\omega, x, u+t)$.

Let $f$ be a measurable function on $\Omega \times X \times \mathbb{R}$ such that

$$
f(\omega, x, u)=f\left(T_{\left(x^{\prime}, x\right)} \omega^{\prime}, x^{\prime}, u-\log \delta\left(x, x^{\prime}\right)\right)
$$

for any $\left(x, x^{\prime}\right) \in \mathscr{G}$ and any $\left(\omega, \omega^{\prime}\right) \in R_{\Omega}$. Upon taking $x=x^{\prime}$, we get

$$
f(\omega, x, u)=f\left(\omega^{\prime}, x, u\right)
$$

for any $\left(\omega, \omega^{\prime}\right) \in R_{\Omega}$. Since $R_{\Omega}$ is ergodic, there exists a measurable function $f_{1}$ on $X \times \mathbf{R}$ such that

$$
f(\omega, x, u)=f_{1}(x, u)
$$

for almost all $(\omega, x, u)$. Hence (6.9) is equivalent to

$$
f_{1}(x, u)=f_{1}\left(x^{\prime}, u-\log \delta\left(x, x^{\prime}\right)\right.
$$


for almost all $\left(x, x^{\prime}\right)$ and $u \in \mathbf{R}$. Since $\mathscr{G}$ is a type $I I I_{\lambda}$ equivalence relation, there exists a measurable map $\phi$ from $X \times \mathbf{R}$ onto $[0,-\log \lambda)$ such that $(1) \phi(x, u)=$ $\phi\left(x^{\prime}, u-\log \delta\left(x, x^{\prime}\right)\right)$ for a.e. $(x, u)$. (2) For any function $\xi$ on $X \times \mathbf{R}$ with $\xi(x, u)=\xi\left(x^{\prime}, u-\log \delta\left(x, x^{\prime}\right)\right)$ for a.e. $(x, u)$, there exists a measurable function $\eta$ on $[0,-\log \lambda)$ such that $\xi(x, u)=\eta(\phi(x, u))$ for a.e. $(x, u)$. (3) If $H_{t}$ (resp. $G_{t}$ ) is a flow on $X \times \mathbf{R}$ (resp. on $[0,-\log \lambda)$ ) defined by

$$
\begin{aligned}
H_{t}(x, u) & =(x, u+t) \quad((x, u) \in X \times \mathbf{R}) \\
\left(\operatorname{resp} . G_{t} u\right. & =u+t \quad \bmod \{-\log \lambda\}),
\end{aligned}
$$

then $\phi \circ H_{t}=G_{t}^{\circ} \phi$. Hence, to the above function $f_{1}$, there exists a measurable function $\eta$ on $[0,-\log \lambda)$ such that $f_{1}(x, u)=\eta(\phi(x, u))$ for a.e. $(x, u)$. This shows that, if $\theta$ denotes a measurable map from $\Omega \times X \times \mathbf{R}$ onto $[0,-\log \lambda)$ given by $\theta(\omega, x, u)=\phi(x, u)$, then, for any measurable function $f$ on $\Omega \times X \times \mathbf{R}$ of the above type, there exists a measurable function $\eta$ on $[0,-\log \lambda)$ such that $f=\eta \circ \phi$. Moreover, it is easy to see that $G_{t} \circ \theta=\theta \circ F_{t}$ and that $\theta(\omega, x, u)=$ $\theta\left(T_{\left(x^{\prime}, \imath\right)} \omega^{\prime}, x^{\prime}, u-\log \delta\left(x, x^{\prime}\right)\right)$. From Lemma 3 in [Hm], or rather, from a slight adaptation of Lemma 3 in [Hm], it follows that the Poincaré flow of $R_{\Omega \times X}$ is the flow $G_{t}$ on $[0,-\log \lambda)$. Therefore, the crossed product $M \times{ }_{a} \mathscr{G}$ is a factor of type $I I I_{\lambda}$.

Let us consider one more example, which is due to Hamachi.

Example 6.10. Let $(X \mu)$ be the infinite direct product measure space

$$
(X, \mu)=\prod_{n=1}^{\infty}\left(\{0,1,2\},\left\{\frac{1}{1+\varepsilon_{1}+\varepsilon_{2}}, \frac{\varepsilon_{1}}{1+\varepsilon_{1}+\varepsilon_{2}}, \frac{\varepsilon_{2}}{1+\varepsilon_{1}+\varepsilon_{2}}\right\}\right)
$$

built from one measure space $\{0,1,2\}$ with $\left\{\frac{1}{1+\varepsilon_{1}+\varepsilon_{2}}, \frac{\varepsilon_{1}}{1+\varepsilon_{1}+\varepsilon_{2}}, \frac{\varepsilon_{1}}{1+\varepsilon_{1}+\varepsilon_{2}}\right\}$ as its probability distribution, where $0<\varepsilon_{1}, \varepsilon_{2}<1$ and $\left\{\log \varepsilon_{1}, \log \varepsilon_{2}\right\}$ is a rationally independent set. As in the previous example, we introduce an equivalence relation on $X$ by saying that $x=\left(x_{i}\right)_{i=1}^{x}$ is equivalent to $x^{\prime}=\left(x_{l}^{\prime}\right)_{i=1}^{x}$ if $x_{t}=x_{l}^{\prime}$ for all but a finite number of $i$. Let $\mathscr{G}$ be the graph of this equivalence relation. Then $\mathscr{G}$ is known to be a type $I I_{1}$ ergodic orbitally discrete principal measured groupoid. It is also known that $\left\{\delta\left(x, x^{\prime}\right):\left(x, x^{\prime}\right) \in \mathscr{G}\right\}$ coincides with a set $\left\{\varepsilon_{1}^{n} \varepsilon_{2}^{m}: n, m \in \mathbf{Z}\right\}$. We put $G=\left\{\log \delta\left(x, x^{\prime}\right):\left(x, x^{\prime}\right) \in \mathscr{G}\right\}$, which is a countable dense subgroup of $\mathbf{R}$. Suppose that $\left\{F_{t}\right\}_{t \in \mathbf{R}}$ is a $\mu_{Z}$-preserving ergodic flow on a Lebesgue space $\left(Z, \mu_{Z}\right)$. To this flow, we associate another flow $\left\{\widetilde{F}_{t}\right\}_{t \in \mathbf{R}}$ on a measure space $(\Omega, m)=\left(Z \times \mathbf{R}, \mu_{Z} \times e^{u} d u\right)$ given by

$$
\left.\widetilde{F}_{t}(z, u)=\left(F_{t} z, u+t\right) \quad((z, u) \in \Omega)\right) .
$$

It is apparent that $\left\{\widetilde{F}_{t}\right\}_{t \in G}$ is a countable group of not necessarily ergodic free transformations of type $I I$. Thus the injective von Neumann algebra $M_{0}$ derived 
from this system is of type $I I$. For each $\left(x, x^{\prime}\right) \in \mathscr{G}$, define an automorphism $T_{\left(x, x^{\prime}\right)}$ on $\Omega$ by

$$
T_{\left(x, x^{\prime}\right)}(z, u)=\left(z, u-\log \delta\left(x, x^{\prime}\right)\right) .
$$

Since $T_{\left(x, x^{\prime}\right)}$ commutes with $\widetilde{F}_{t}$ for all $\left(x, x^{\prime}\right) \in \mathscr{G}$ and any $t \in G, T_{\left(x, x^{\prime}\right)}$ belongs to $N[G]$. We denote by $\alpha_{\left(x, x^{\prime}\right)}$ the automorphism of $\mu_{0}$ induced by $T_{\left(x, x^{\prime}\right)}$. Thus we get an action $\left(\mathscr{G},\left\{M(x)=M_{0}\right\},\left\{\alpha_{\left(x, x^{\prime}\right)}\right\}\right)$ of $\mathscr{G}$. By Theorem 6.7 , the crossed product $\mathcal{M} \times{ }_{\alpha} \varphi$ is isomorphic to $W^{*}\left(R_{\Omega \times X}\right)$. Let $\mathfrak{B}_{0}$ be a von Neumann subalgebra of $L^{\nsim}(\Omega \times X, m \times \mu)$ consisting of functions $f$ with the following property:

$$
f(z, u, x)=f\left(F_{t} z, u+t-\log \delta\left(x^{\prime}, x\right), x^{\prime}\right)
$$

for a.e. $(z, u) \in \Omega$, a.e. $\left(x, x^{\prime}\right) \in \mathscr{G}$ and any $t \in G$. Then, from Theorem 5.1 in [Ha2], the center $\mathscr{E}\left(W^{*}\left(R_{\Omega \times X}\right)\right)$ of $W^{*}\left(R_{\Omega \times X}\right)$ is the algebra consisting of operators of the form $M_{r}(f)\left(f \in \mathfrak{B}_{0}\right)$, where

$$
\left\{M_{r}(f) \eta\right\}\left((\omega, x),\left(\omega^{\prime}, x^{\prime}\right)\right)=f(\omega, x) \eta\left((\omega, x),\left(\omega^{\prime}, x^{\prime}\right)\right)
$$

for $\left((\omega, x),\left(\omega^{\prime} x^{\prime}\right)\right) \in R_{\Omega \times X}$ and $\eta \in L^{2}\left(R_{\Omega \times X}\right)$. We assert that $\mathfrak{B}_{0}=\mathbb{C}$. Indeed, let us take a function $f$ satisfying (6.11). By taking $t=0$, we get

$$
f(z, u, x)=f\left(z, u-\log \delta\left(x^{\prime}, x\right), x^{\prime}\right) .
$$

Since $G$ is of type $I I I_{1}$, there exists a measurable function $f_{1}$ on $Z$ such that $f(z, u, x)=f_{1}(z)$ for a.e. $(z, u, x)$. Hence (6.11) is the same as

$$
f_{1}(z)=f_{1}\left(F_{t} z\right)
$$

for any $t \in G$. Since $\left\{F_{t}\right\}$ is ergodic and measurable, $f_{1}$ is constant. Accordingly, $f$ is constant. This shows that $W^{*}\left(R_{\Omega \times X}\right)$ is a factor.

We would like to compute the Poincaré flow of the equivalence relation $R_{\Omega \times X}$. For this, suppose that $g$ is a function on $\left(\Omega \times X \times \mathbb{R}, m \times \mu \times e^{u} d u\right)$ such that

$$
g(z, u, x, v)=g\left(F_{t} z, u+t-\log \delta\left(x^{\prime}, x\right), x^{\prime}, v-t\right) .
$$

From this point on, one can follow the proof of Theorem 4 in $[\mathrm{Hm}]$ in order to conclude that the Poincaré flow is isomorphic to the given flow $\left\{F_{t}\right\}$. Hence we have shown that $M \times{ }_{a} G$ is an injective factor of type $I I I_{0}$.

\section{References}

[C1] Connes, A.. Une classification des facteurs de type III, Ann, Sci. École Norm. Sup., 6 (1973), 133-252.

[C2] Sur la thćorie non commutative de l'intégration, Lecture Notes in Math., Springer-Verlag, 725 (1979), 19-143.

[C3] On the spatial theory of von Neumann algebras, J. Functional Analysis, 35 (1980), 153-164. 
[C\&T] Connes, A. and Takesaki, M., The flow of weights of factors of type III, Tohoku Math. J., 29 (1977), 473-575.

[D] Digernes, T., Duality for weights on covariant systems and its applications, Dissertation at UCLA (1975).

[F\&M1] Feldman, J. and Moore, C., Ergodic equivalence relations, cohomology and von Neumann algebras I, Trans. Amer. Math. Soc., 234 (1977), 289-324.

[F\&M2] _ Ergodic equivalence relations, cohomology and von Neumann algebras II, Trans. Amer. Math. Soc., 234 (1977), 325-359.

[H1] Haagerup, U., The standard form of von Neumann algebras, Math. Scan., 37 (1975), $271-283$.

[H2] On the dual weight for crossed products of von Neumann algebras I, Math. Scan., 43 (1978), 99-118.

[H\&S] Haagerup, U. and Størmer, E.. Equivalence of normal states on von Neumann algebras and the flow of weights, To appear in Adv. in Math.

[Ha1] Hahn, P., Haar measure for measure groupoids, Trans. Amer. Math. Soc., 242 (1978), $1-33$.

[Ha2] The regular representations of measure groupoids. Trans. Amer. Math. Soc., 242 (1978), 34-72.

[Hm] Hamachi, T., The normalizer group of an ergodic automorphism of type III and the commutant of an ergodic flow, J. Functional Analysis, 40 (1981), 387-403.

[H\&O] Hamachi, T. and Osikawa, M., Ergodic group of automorphisms and Krieger's theorem, Keio Univ., Seminar on Math. Sci., No. 3 (1981).

[J\&T] Jones, V.F.R. and Takesaki. M., Actions of abelian compact groups on semifinite injective factors, Acta Math.. 153 (1984), 213-258.

[K] Kastler, D., On A. Connes' noncommutative integration theory, Comm. Math. Phys., 85 (1982), 99-120.

[Ko] Kosaki. H., Canonical $L^{p}$-spaces associated with arbitrary abstract von Neumann algebras, Dissertation at UCLA (1980).

[Kr1] Krieger, W.. On constructing non-*-isomorphic hyperfinite factors of type III, J. Functional Analysis, 6 (1970), 97-109.

[Kr2] On ergodic flows and isomorphism of factors, Math. Ann., 223 (1976), 19-70.

[M1] Mackey, G.W., Borel structure in groups and their duals, Trans. Amer. Math. Soc., 85 (1957), 265-311.

[M2] Ergodic theory and virtual groups, Math. Ann., 166 (1966), 187-207.

[N\&T] Nakagami, Y. and Takesaki, M., Duality for crossed products of von Neumann algebras. Lecture Notes in Math., Springer-Verlag, 731 (1979).

[P\&T] Pedersen, G.K. and Takesaki, M., The Radon-Nikodym theorem for von Neumann algebras, Acta Math., 130 (1975), 53-87.

[R] Ramsay, A., Virtual groups and group actions, Adv. in Math., 6 (1971), 253-322.

[Re] Renault. J., A groupoid approach to $C^{\prime}$-algebras, Lecture Notes in Math.. SpringerVerlag. 791 (1980).

[S1] Sauvageot, J.L., Sur le type du produit croisé d'une algébres de von Neumann par un groupe localement compact, Bull. Soc. Math. France, 105 (1977), 349-368.

[S2] - Produits tensoriels de $\mathscr{Z}$-modules, Publ. Univ. P. \& M. Curie, $n^{\circ} 23$ (1980).

[S3] , Produit tensoriels de $\mathscr{L}$-modules et application, Lecture Notes in Math., SpringerVerlag, 1132 (1983), 468-485.

[S4] - Sur le produit tensoriel relatif d'espace de Hilbert. J. Operator Theory, 9 (1983), 237-252.

[Su] Sutherland, C., The direct integral theory of weights, and the Plancherel formula, Dissertation at UCLA (1973).

[S\&T1] Sutherland, C. and Takesaki, M., Actions of amenable groups and groupoids on semifinite injective von Neumann algebras, R.I.M.S. Kyoto Univ., 21 (1985), 1087-1120. 
[S\&T2] Sutherland, C. and Takesaki, M., Actions of discrete amenable groups on injective factors of type $I I I_{\lambda}, \lambda \neq 1$, Pacific J. Math., 137 (1989), 405-443.

[T1] Takcsaki, M., Tomita's theory of modular Hilbert algebras and its application, Lecture Notes in Math. Springer-Verlag, 128 (1970).

[T2] Duality for crossed products and the structure of von Neumann algebras of type III, Acta Math., 131 (1973), 249-310.

[T3] , Theory of operator algebras I, Springer-Verlag 1979.

[T4] The structure of operator algebras (in Japanese), Iwanami-shoten. 1983.

[Y1] Yamanouchi. T., Duality for actions and coactions of measured groupoids on von Neumann algebras. Dissertation at UCLA (1990).

[Y2] . Fixed point algebras of groupoid actions and coactions, in preparation. 